\title{
A survey of $\mathrm{OH}$ masers towards high mass protostellar objects ${ }^{\star \star \star}$
}

\author{
K. A. Edris ${ }^{1,2}$, G. A. Fuller ${ }^{1}$, and R. J. Cohen ${ }^{3}$ \\ 1 The University of Manchester, School of Physics and Astronomy, Sackville Street Building, PO Box 88, \\ Manchester M60 1QD, UK \\ e-mail: G.Fuller@manchester . ac.uk \\ 2 Al-Azhar University, Faculty of Science, Astronomy Department, PO Box 11884, Naser City, Cairo, Egypt \\ 3 The University of Manchester, Jodrell Bank Observatory, Macclesfield, Cheshire SK11 9DL, UK
}

Received 21 August 2006 / Accepted 18 December 2006

\begin{abstract}
Context. Masers are important tracers of the early evolution of young high mass stars, but the relationship between different types of maser and the evolutionary state of the exciting source remains unclear.

Aims. To determine whether $\mathrm{OH}$ masers are common towards candidate high mass protostellar objects.

Methods. We present a survey of $\mathrm{OH}$ maser emission towards a sample of high mass protostellar objects made using the Nançay and GBT telescopes.

Results. $\mathrm{OH}$ maser emission was detected towards 63 objects with 36 new detections. There are 56 star-forming regions and $7 \mathrm{OH} / \mathrm{IR}$ candidates. Nearly half of the detected sources have $\mathrm{OH}$ flux densities $\lesssim 1 \mathrm{Jy}$. There is no evidence that sources with $\mathrm{OH}$ masers have a different range of luminosities from the non-maser sources. The results of this survey are compared with previous $\mathrm{H}_{2} \mathrm{O}$ and class II $\mathrm{CH}_{3} \mathrm{OH}$ maser observations of the same objects. Some of the detected sources are only associated with $\mathrm{OH}$ masers and some sources are only associated with the $1720 \mathrm{MHz} \mathrm{OH}$ maser line. The velocity range of the maser emission suggests that the water maser sources may be divided into two groups. The detection rates and velocity range of the $\mathrm{OH}$ and Class $\mathrm{II} \mathrm{CH}_{3} \mathrm{OH}$ masers support the idea that there is a spatial association of the $\mathrm{OH}$ and Class II $\mathrm{CH}_{3} \mathrm{OH}$ masers. The sources span a wide range in $R$, the ratio of the methanol maser peak flux to $\mathrm{OH} 1665 \mathrm{MHz}$ maser peak flux, however there are only a few sources with intermediate values of $R, 8<R<32$, which has characterised previous samples. The majority of the sources are either methanol-favoured or OH-favoured. Sources which have masers of any species, OH, water or methanol, have redder [100 $\mu \mathrm{m}-12 \mu \mathrm{m}]$ IRAS colours than those without masers. However, there is no evidence for different maser species tracing different stages in the evolution of these young high mass sources.

Conclusions. The detection of $\mathrm{OH}$ masers towards $26 \%$ of a sample of 217 sources should remove any doubt about the existence of $\mathrm{OH}$ maser emission towards these objects or this early evolutionary stage. Previous observations which have shown that the $\mathrm{OH}$ maser emission from similar sources traces the circumstellar disks around the objects. This combined with the sensitivity of the $\mathrm{OH}$ emission to the magnetic field, make the newly detected sources interesting candidates for future follow-up at high angular resolution.
\end{abstract}

Key words. masers - stars: formation - ISM: molecules - ISM: HII regions

\section{Introduction}

Compact HII regions, poorly collimated bipolar molecular outflows and circumstellar disks are signs of the existence of massive protostars (e.g. Garay \& Lizano 1999; Churchwell 2002). Maser emission is also found to be associated with these objects (e.g. Garay \& Lizano 1999; Edris et al. 2005) with $\mathrm{OH}$, $\mathrm{H}_{2} \mathrm{O}$ and $\mathrm{CH}_{3} \mathrm{OH}$ the three most widespread types of maser associated with these regions. These species have been used as probes of star-forming regions as their maser emission provide unique information on these dense dusty regions (e.g. Cohen 1989, and references therein). Observations of $\mathrm{H}_{2} \mathrm{O}$ and Class II $\mathrm{CH}_{3} \mathrm{OH}$ masers have shown that both maser types are signposts of high mass star formation in very early evolutionary stages

* Figures 17-20 and Appendix A are only available in electronic form at http://www . aanda.org

$\star \star$ Data files used for Figs. 19 and 20 are only available in electronic form at the CDS via anonymous ftp to

cdsarc.u-strasbg.fr $(130.79 .128 .5)$ or via

http://cdsweb.u-strasbg.fr/cgi-bin/qcat?J/A+A/465/865
(Beuther et al. 2002a; Szymczak et al. 2000a, hereafter SHK2000; Palla et al. 1991, hereafter P91).

On the other hand $\mathrm{OH}$ masers are known to be associated with an advanced stage of the appearance of UCHII region (e.g. Garay \& Lizano 1999, and references therein). Models of these $\mathrm{OH}$ masers assume that the maser arises in the compressed shell between the shock and ionisation fronts around the HII region (Elitzur \& De Jong 1978). However in a survey, Caswell (1983) found a large proportion of $\mathrm{OH}$ masers have no closely related prominent HII regions. In some molecular outflow sources, $\mathrm{OH}$ masers have been mapped with high angular resolution and found to be associated with an earlier stage of molecular outflows and circumstellar disks (Cohen et al. 1984; Brebner 1988; Cohen et al. 2003; Edris et al. 2005). Since the masers can be observed with high spatial, and spectral, resolution they can probe the inner regions of these sources. $\mathrm{OH}$ masers also provide the possibility of measuring the magnetic fields in these regions.

With this in mind, and to form a more complete picture of the relationship between maser emission and the evolution of high mass protostars, 217 high mass protostellar object (HMPO) candidates have been surveyed for $\mathrm{OH}$ maser emission using the 
Table 1. IRAS colour index selection criteria proposed for different objects by different authors. $[x-y] \equiv \log \left(F_{x}\right) / \log \left(F_{y}\right)$. References: (1) Emerson (1987); (2) Wouterloot \& Walmsley (1986); (3) Wood \& Churchwell (1989); (4) Braz et al. (1989); (5) P91; CMC $\equiv$ Compact Molecular Cloud; (6) Present work (Sect. 5.2).

\begin{tabular}{llllll}
\hline \hline Class of object & {$[25-12]$} & {$[60-25]$} & {$[100-60]$} & {$[60-12]$} & Ref. \\
\hline Cores & $0.4-1.0$ & $0.4-1.3$ & $0.1-0.7$ & - & 1 \\
$\mathrm{H}_{2} \mathrm{O}$ maser & $0.5-1.1$ & $0.4-1.7$ & $-0.1-0.5$ & - & 2 \\
UCHII regions & $>0.6$ & - & - & $>1.3$ & 3 \\
Bright IRAS & $0.5-1.2$ & $0.6-1.6$ & $0.0-0.6$ & - & 4 \\
$\mathrm{CMC}+\mathrm{UCHII}$ & $\geq 0.57$ & $0.61-1.74$ & $0.087-0.52$ & $\geq 1.3$ & 5 \\
$\mathrm{OH}$ maser & $>1.2$ & - & - & $>2.2$ & 6 \\
\hline
\end{tabular}

Nançay radio telescope ${ }^{1}$ and the NRAO Green Bank Telescope ${ }^{2}$ (GBT). The aims of this survey are:

- to determine whether $\mathrm{OH}$ masers are associated with these objects;

- to investigate the relationship between the $\mathrm{OH}$ and $\mathrm{H}_{2} \mathrm{O}$ and/or $\mathrm{CH}_{3} \mathrm{OH}$ maser emission and whether the masers are related to the evolutionary stage of these objects or represent different regions of the star formation core;

- to identify sources for further study at high angular resolution.

The description of the sample is given in Sect. 2 and the details of the observations are given in Sect. 3. In Sect. 4 we report the results of the survey while Sect. 5 presents some detection statistics. In Sect. 6 we discuss the interpretation while the conclusions are drawn in Sect. 7.

\section{The sample}

Different selection criteria have been proposed by many authors to use the IRAS point source catalogue to identify massive young sources (Table 1, and references therein). The sample of sources in this present survey is drawn from the sample of Sridharan et al. (2002, hereafter S02) and Molinari et al. (1996, hereafter M96). These two samples are believed to contain massive sources in a very early stage of evolution prior to the forming of UCHII regions. M 96 divided their sample into two subsamples, a High sample and a Low sample. The S02 sample and the High sub-sample of M 96 satisfy the colour selection criteria of Wood \& Churchwell (1989, hereafter WC89) for UCHII regions. However the sources in these two samples (and also the M 96 Low sub-sample) are not known to be associated with detectable HII regions. M96 suggest that their Low sub-sample comprises objects which are in a different evolutionary stage from those in their High sub-sample, and therefore also from the S02 sample. The two samples (S02 and M 96) have sources with flux densities brighter than $90 \mathrm{Jy}$ at $60 \mu \mathrm{m}$. Figure 1 shows the $[25-12]^{3}$ versus [60-12] colour-colour diagram, indicating the location of the High, Low and S02 samples, for the whole sample observed here. Figure 1 also shows the results of this survey

1 The Nançay Radio Observatory is the Unité scientifique de Nançay of the Observatoire de Paris, associated as Unité de Service et de Recherche (USR) No. 704 to the French Centre National de la Recherche Scientifique (CNRS). The Nançay Observatory also gratefully acknowledges the financial support of the Conseil Régional de la Région Centre in France.

2 The National Radio Astronomy Observatory (NRAO) is a facility of the National Science Foundation operated under cooperative agreement by Associated Universities, Inc.

${ }^{3}[x-y]$ indicates $\log \left(F_{x}\right) / \log \left(F_{y}\right)$.

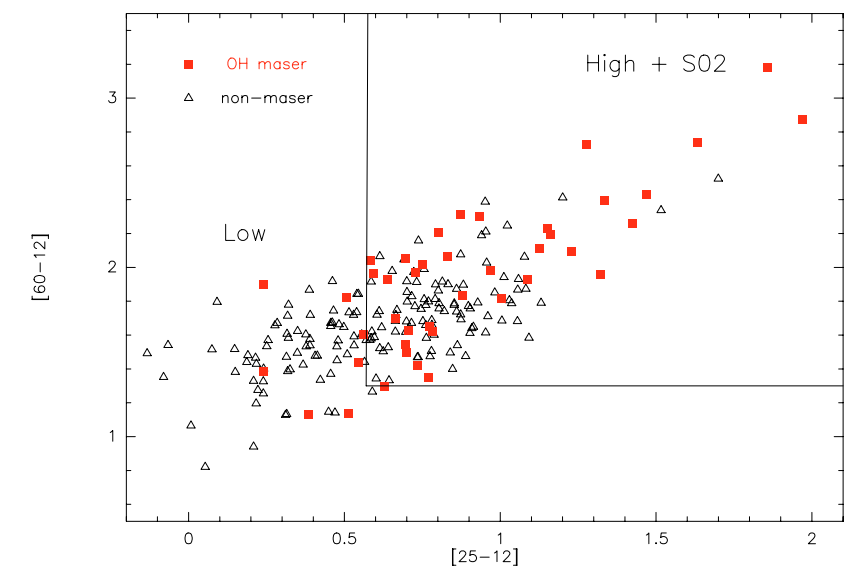

Fig. 1. The [60-12] vs. [25-12] two colour plot for the objects searched for $\mathrm{OH}$ maser emission (except the seven objects cataloged in Table 2 as offset sources). The box identifies the High and S02 samples which contain sources which agree with WC89 criteria to select UCHII region candidates. The sources of the Low sample are outside this box. $\mathrm{OH}$ maser sources are plotted as filled squares, while non- $\mathrm{OH}$ masers are shown as open triangles.

(Sect. 4); the detected sources of $\mathrm{OH}$ maser emission are marked with filled squares.

Observations show evidence of dense molecular gas associated with the majority of sources in the sample. The sources in the S02 sample have all been detected in CS $J=2-1$ ( $\mathrm{S} 02$ ) while ammonia was detected towards $80 \%$ and $45 \%$ of the High and Low sub-samples respectively of M 96. Further details about the sources and their selection criteria can be found in S02 and M 96 and references therein.

Combining the M 96 and S02 samples, and accounting for the 15 sources in common, results in a sample of 217 of HMPO candidates which have been observed here. The vast majority of the sources in the sample have luminosities in the range $\sim 10^{3} L_{\odot}$ to $10^{5.5} L_{\odot}$. However distance uncertainties affect the luminosity estimates of individual sources and are likely to explain a few sources with apparently with much lower luminosities. Although these objects have been previously systematically surveyed for water and methanol masers, only a handful have previously been searched for $\mathrm{OH}$ masers.

\subsection{Water and methanol masers in the sample}

$\mathrm{S} 02$ searched their sample for $22 \mathrm{GHz} \mathrm{H}_{2} \mathrm{O}$ and $6.7 \mathrm{GHz} \mathrm{CH}_{3} \mathrm{OH}$ masers using the Effelsberg $100 \mathrm{~m}$ telescope. The detected sources $\left(29 \mathrm{H}_{2} \mathrm{O}\right.$ and $26 \mathrm{CH}_{3} \mathrm{OH}$ masers) were mapped with the Very Large Array (VLA) by Beuther et al. (2002a). The M 96 sample had already been surveyed for $\mathrm{H}_{2} \mathrm{O}$ maser by $\mathrm{P} 91$ with the Medicina $32 \mathrm{~m}$ telescope and most of the sources were searched for $6.7 \mathrm{GHz} \mathrm{CH}_{3} \mathrm{OH}$ maser emission by Szymczak et al. (2000a) using the Torun $32 \mathrm{~m}$ radio telescope. Towards the M 96 sample $40 \mathrm{H}_{2} \mathrm{O}$ and $26 \mathrm{CH}_{3} \mathrm{OH}$ maser sources have been reported. Therefore water and methanol masers were found towards $36 \%$ and $21 \%$, respectively, of the 217 sources.

\subsection{Outflows in the sample}

Beuther et al. (2002b) searched 26 sources from the S02 sample at a spatial resolution of $11^{\prime \prime}$ for bipolar molecular outflows. The signature of outflow was found towards 21 sources of them. The other five sources showed confusing morphology but have 
strong line wings. More recently, Zhang et al. (2005) studied this phenomenon towards 69 sources of the M 96 sample. Mapping in the $\mathrm{CO} J=2-1$ line, they identified 39 molecular outflows. Therefore, among 95 sources systematically searched out of the total of 217 objects, 60 sources show clear evidence of bipolar molecular outflows. This gives a $63 \%$ detection rate which indicates that molecular outflows are very common in these regions.

\section{Observations}

\subsection{Nançay observations}

Observations using the Nançay radio telescope were performed between July 2002 and June 2003. At $18 \mathrm{~cm}$ the telescope has a beamsize of $3.5^{\prime} \times 19^{\prime}(\mathrm{RA} \times \mathrm{Dec})$. We simultaneously observed the four $\mathrm{OH}$ transitions at 1665, 1667, 1612 and $1720 \mathrm{MHz}$ in both left and right circular polarizations. The 8192 channel autocorrelator was configured into eight banks of 1024 channels. Each bank had a total bandwidth of $1.5625 \mathrm{MHz}$ yielding velocity resolutions of $0.284,0.275,0.274$ and $0.266 \mathrm{~km} \mathrm{~s}^{-1}$ at $1612,1665,1667$ and $1720 \mathrm{MHz}$, respectively. Inband frequency-switching was used during these observations. The total integration time per sources was $18 \mathrm{~min}$, giving a typical noise level in a single polarisation of about $50 \mathrm{mJy}$. A total useable velocity range of about $\pm 160 \mathrm{~km} \mathrm{~s}^{-1}$ was covered towards each source. The radial velocities were measured with respect to the local standard of rest (LSR). The spectral bandwidth was centred at the molecular gas velocity of the observed source, as given by M 96 and S02. The NAnçay Preprocessing Software (NAPS) program was used for the initial data processing and eliminating bad integrations, and integrating the whole cycles of each scan. The data were then imported into CLASS for further processing. In CLASS, the spectra were FOLDed to remove ripples coming from the frequency-switching technique and finally the spectral plots were produced.

\subsection{GBT observations}

The Green Bank Telescope (GBT) was used to re-observe the sources detected by Nançay in order to: (1) observe them with higher spectral resolution; (2) decrease the contamination due to Nançay's large beam size $\left(3.5^{\prime} \times 19^{\prime}\right.$, comparing to $\sim 8^{\prime}$ of GBT); and (3) make small maps to determine whether the masers were associated with these IRAS sources or offset from them. The GBT was also used to observe a small set of sources we did not have enough time to observe with Nançay. The observations were carried out from 18 to 23 May 2003. The $12.5 \mathrm{MHz}, 9$ level, 8 sampler correlator setting was used to observe all four $\mathrm{OH}$ lines $(1665,1667,1612$ and $1720 \mathrm{MHz})$ in both senses of circular polarizations. After confirming the presence of an $\mathrm{OH}$ maser towards the IRAS position of a source, most of the sources were mapped with small 3 arcmin sampled maps, typically $3 \times$ 3 pixels in size, to determine the position of the peak emission. For the majority of sources, where the position of the $\mathrm{OH}$ masers was consistent with the IRAS position, a higher resolution spectrum (with a velocity channel width of $\sim 0.07 \mathrm{~km} \mathrm{~s}^{-1}$ ) was then obtained towards the IRAS position. The typical noise level in these high resolution spectra as about $0.1 \mathrm{Jy}$. For the high resolution spectra, the $12.5 \mathrm{MHz}, 3$ level, 8 sampler mode was used to obtain four times higher resolution and covering all four $\mathrm{OH}$ lines in both senses of circular polarizations. Frequency switching was used during these observation. The typical system temperature was $\sim 20 \mathrm{~K}$ resulting in a typical noise level of $50 \mathrm{mJy}$ and $150 \mathrm{mJy}$ in the low and high resolution spectrum respectively. Note that sources observed at Nançay but not detected were not reobserved at GBT.

\section{Results}

Combining the results from both Nançay and GBT, a total of 63 sources show $\mathrm{OH}$ maser emission, defined as bright and narrow polarized lines, in one or more of the $\mathrm{OH}$ transitions. Of these 36 have not been reported before.

The nature of the $\mathrm{OH}$ maser emission provides the opportunity to distinguish between sources which have same colours as HMPOs but are in fact late type stars around which $\mathrm{OH}$ masers are also known to occur (e.g. Cohen 1989). Towards evolved stars the $1612 \mathrm{MHz}$ line spectrum usually shows double-peaked profile with sharp external edges and smooth internal ones with the two peaks separated by 15 to $40 \mathrm{~km} \mathrm{~s}^{-1}$ (Cohen 1989). Of the sources detected here 7 have $1612 \mathrm{MHz}$ masers which indicate that they are $\mathrm{OH} / \mathrm{IR}$ candidates.

The 63 sources detected include 57 detected at both Nançay and GBT, and 6 which were detected at Nançay but not observed at the GBT. We categorize these 6 sources as not confirmed, and do not consider them in our subsequent analysis.

The association of the maser emission and the IRAS source that we searched towards is confirmed by GBT maps for the first 46 sources in the table. Only these 46 objects are considered in the IRAS-related statistical analysis that follows. Thirty nine of these IRAS-associated sources are typical starforming regions with masers strong in the main lines, 1665 and $1667 \mathrm{MHz}$. One source, IRAS 18463+0052, is associated with an $\mathrm{OH} / \mathrm{IR}$ star. The remaining 6 sources have $\mathrm{OH}$ masers which are not typical of star-forming regions; they show maser emission in one of the satellite lines only (see Sect. 6.4). There are remaining 11 confirmed sources with $\mathrm{OH}$ maser emission offset by $>2^{\prime}$ from the IRAS position are cataloged as offset sources in Table 2. They include $4 \mathrm{OH} / \mathrm{IR}$ candidates and 7 sources with $\mathrm{OH}$ spectra characteristic of star-forming regions.

The 57 confirmed maser sources and their $\mathrm{OH}$ line parameters are listed in Table 2. Column 1 gives the IRAS name of the source, Cols. 2 and 3 the maser position, where measured, with the uncertainties, Col. 4 the frequency and circular polarization of the maser line, with either L or R referring to lefthand or righthand circular polarisation respectively, Col. 5 the feature central velocity (relative to the LSR), Cols. 6 and 7 the velocity interval at zero intensity, Col. 8 the peak flux density in Jy, Col. 9 rms noise, Col. 10 the ratio of the $\mathrm{CH}_{3} \mathrm{OH}$ peak flux density to the $\mathrm{OH}$ peak flux density, R, as defined by Caswell (1998), and Col. 11 some comments.

Figure 2 shows an example set of $\mathrm{OH}$ maser spectra and map from the GBT (IRAS 18144-1723, left panels). The figure also shows an example of one of the sources cataloged as offset because their map reveal that the emission is not consistent with the IRAS position (IRAS $18540+0220$, right panels). Many of the sources with maser emission, for example IRAS 17527-2339 (Fig. 19), show "conjugate" behaviour with one of the satellite lines in emission while the other is in absorption (Elitzur 1976). The maps and spectra of all the $\mathrm{OH}$ detected sources are shown in Figs. 17 and 19 respectively. The maps and spectra of the sources with maser emission offset from the IRAS position by $>2^{\prime}$ are separated in to Figs. 18 and 20 respectively.

A further 79 sources were detected in thermal emission and/or absorption. They are listed in Table 3 along with the 75 sources which were not detected in our observations. Table 3 also list the 6 sources categorized as not confirmed maser 
Table 2. The detected $\mathrm{OH}$ maser lines and their parameters. The offset star forming region and $\mathrm{OH} / \mathrm{IR}$ candidates are separated at the end of this table. The listed positions are measured from GBT observations except some positions taken from higher resolution observations of Argon et al. (2000) and Edris et al. (2005) (Refs. 1 and 2 in the table respectively).

\begin{tabular}{|c|c|c|c|c|c|c|c|c|c|c|}
\hline \multirow[t]{2}{*}{ IRAS Name } & \multicolumn{2}{|c|}{ Position } & \multirow[t]{2}{*}{ Frequency } & \multicolumn{3}{|c|}{ Velocity at } & \multirow{2}{*}{$\begin{array}{c}\text { Flux at } \\
S_{\text {peak }} \\
\text { Jy }\end{array}$} & \multirow{2}{*}{$\begin{array}{c}\mathrm{rms} \\
\mathrm{Jy}\end{array}$} & \multirow[t]{2}{*}{$R$} & \multirow[t]{2}{*}{ Ref./Notes } \\
\hline & $\begin{array}{c}\mathrm{RA}(\mathrm{J} 2000) \\
\mathrm{h} \mathrm{m} \mathrm{s}\end{array}$ & $\begin{array}{c}\operatorname{Dec}(\mathrm{J} 2000) \\
\circ, \prime \prime\end{array}$ & & $V_{\text {peak }}$ & $\frac{V_{\min }}{\mathrm{km} \mathrm{s}^{-1}}$ & $V_{\max }$ & & & & \\
\hline $05137+3919$ & $051712.8 \pm 2.2$ & $392205 \pm 38$ & $1665 \mathrm{R}$ & -21.58 & -24.5 & -21.2 & 2.20 & 0.40 & & \\
\hline \multirow[t]{3}{*}{$05274+3345$} & $053106.4 \pm 1.4$ & $334727 \pm 19$ & $1665 \mathrm{R}$ & -3.61 & -5 & 0.6 & 1.34 & 0.18 & 70 & \\
\hline & & & $1667 \mathrm{~L}$ & -4.93 & & & 0.14 & 0.04 & & \\
\hline & & & $1612 \mathrm{~L}$ & -3.87 & & & 0.13 & 0.05 & & \\
\hline \multirow[t]{2}{*}{$05358+3543$} & $053913.0 \pm 0.1$ & $354551 \pm 01$ & $1665 \mathrm{~L}$ & -10.88 & -16.5 & -8.5 & 2.82 & 0.47 & 91 & 1 \\
\hline & & & $1667 \mathrm{~L}$ & -10.53 & & & 0.93 & 0.17 & & \\
\hline $05382+3547$ & $054112.9 \pm 1.3$ & $355406 \pm 20$ & $1665 \mathrm{R}$ & -26.83 & -27.0 & -26.5 & 0.50 & 0.13 & 15 & \\
\hline \multirow[t]{3}{*}{$06056+2131$} & $060852.4 \pm 1.6$ & $213406 \pm 23$ & $1665 \mathrm{~L}$ & 10.14 & 3.0 & 11.0 & 3.23 & 0.51 & 6 & \\
\hline & & & $1667 \mathrm{R}$ & 9.44 & & & 0.22 & 0.05 & & off source \\
\hline & & & $1720 \mathrm{R}$ & 3.37 & & & 0.60 & 0.10 & & \\
\hline $17527-2439$ & $175528.3 \pm 1.9$ & $-243636 \pm 27$ & $1665 \mathrm{R}$ & 11.53 & 8.2 & 12.2 & 0.40 & 0.14 & & \\
\hline \multirow[t]{2}{*}{$18018-2426$} & $180453.1 \pm 0.1$ & $-242641 \pm 01$ & $1665 \mathrm{R}$ & 10.84 & 10.0 & 12.0 & 8.10 & 1.83 & & \\
\hline & & & $1667 \mathrm{~L}$ & 11.05 & & & 0.98 & 0.20 & & 1 \\
\hline $18024-2119$ & $180525.6 \pm 2.3$ & $-211459 \pm 19$ & $1665 \mathrm{R}$ & -4.05 & -9.0 & 31.0 & 0.69 & 0.17 & 145 & \\
\hline & & & 1667R & -3.75 & & & 0.38 & 0.10 & & \\
\hline $18048-2019$ & $180744.6 \pm 2.2$ & $-201841 \pm 38$ & $1665 \mathrm{R}$ & 44.36 & 40.0 & 44.7 & 0.34 & 0.08 & 104 & \\
\hline & & & $1667 \mathrm{~L}$ & 40.28 & & & 0.27 & 0.09 & & \\
\hline $18089-1732$ & $181151.4 \pm 0.1$ & $-173129 \pm 01$ & $1665 \mathrm{~L}$ & 32.92 & 31.0 & 36.0 & 10.30 & 1.86 & 6 & 1 \\
\hline & & & $1667 \mathrm{~L}$ & 33.36 & & & 2.00 & 0.32 & & \\
\hline $18090-1832$ & $181147.4 \pm 1.5$ & $-182947 \pm 26$ & $1665 \mathrm{R}$ & 108.9 & 103.0 & 110.0 & 0.70 & 0.08 & 110 & \\
\hline & & & $1667 \mathrm{R}$ & 106.6 & & & 0.49 & 0.08 & & \\
\hline $18102-1800$ & $181304.4 \pm 1.5$ & $-180023 \pm 16$ & $1665 \mathrm{R}$ & 24.40 & 24.0 & 25.0 & 0.42 & 0.10 & 31 & \\
\hline $18144-1723$ & $181726.5 \pm 1.1$ & $-172229 \pm 16$ & $1665 \mathrm{~L}$ & 48.33 & 48.0 & 64.0 & 35.9 & 3.45 & 1 & \\
\hline & & & $1667 \mathrm{~L}$ & 61.75 & & & 4.80 & 0.62 & & \\
\hline $18182-1433$ & $182111.0 \pm 1.0$ & $-143123 \pm 14$ & $1665 \mathrm{~L}$ & 61.55 & 58.0 & 64.0 & 0.72 & 0.16 & 33 & \\
\hline & & & $1667 \mathrm{~L}$ & 62.45 & & & 0.40 & 0.12 & & \\
\hline $18236-1205$ & $182636.2 \pm 1.0$ & $-120454 \pm 14$ & $1665 \mathrm{R}$ & 31.09 & 20.0 & 32.0 & 0.80 & 0.16 & 8 & \\
\hline & $182631.7 \pm 1.0$ & $-120326 \pm 14$ & $1667 \mathrm{~L}$ & 62.45 & & & 0.28 & 0.06 & & \\
\hline $18264-1152$ & $182919.2 \pm 1.1$ & $-115005 \pm 17$ & $1720 \mathrm{~L}$ & 39.31 & 37.5 & 43.5 & 2.30 & 0.55 & & \\
\hline $18278-1009$ & $183037.9 \pm 1.1$ & $-100725 \pm 17$ & $1665 \mathrm{R}$ & 119.7 & 118.0 & 121.0 & 0.42 & 0.09 & 33 & \\
\hline $18290-0924$ & $183146.4 \pm 1.1$ & $-092214 \pm 15$ & $1665 \mathrm{R}$ & 78.33 & 76.0 & 84.0 & 2.30 & 0.41 & 5 & \\
\hline & & & $1667 \mathrm{R}$ & 78.45 & & & 0.34 & 0.90 & & \\
\hline $18310-0825$ & $183336.0 \pm 0.8$ & $-081946 \pm 13$ & $1667 \mathrm{~L}$ & 88.74 & 88.0 & 89.0 & 1.40 & 0.21 & & \\
\hline $18316-0602$ & $183425.9 \pm 1.1$ & $-060001 \pm 16$ & $1665 \mathrm{R}$ & 39.90 & 36.0 & 46.0 & 6.00 & 0.87 & 30 & \\
\hline & & & $1667 \mathrm{~L}$ & 40.41 & & & 3.38 & 0.48 & & \\
\hline $18345-0641$ & $184708.0 \pm 2.8$ & $-021905 \pm 40$ & 1612R & 93.52 & 92.0 & 96.0 & 0.43 & 0.07 & 23 & \\
\hline $18360-0537$ & $183842.2 \pm 1.1$ & $-053625 \pm 16$ & $1665 \mathrm{R}$ & 102.9 & 102.0 & 106.0 & 0.54 & 0.05 & & \\
\hline & $183847.2 \pm 1.2$ & $-053540 \pm 17$ & $1667 \mathrm{~L}$ & 105.3 & & & 0.74 & 0.05 & & \\
\hline $18385-0512$ & $184118.2 \pm 1.0$ & $-050857 \pm 14$ & $1665 \mathrm{R}$ & 24.87 & 21.0 & 30.0 & 1.01 & 0.04 & & \\
\hline $18440-0148$ & $184637.8 \pm 1.0$ & $-014427 \pm 13$ & $1665 \mathrm{R}$ & 101.4 & 99.0 & 110.0 & 6.00 & 0.08 & 0.5 & \\
\hline & & & $1667 \mathrm{R}$ & 102.6 & & & 2.00 & 0.10 & & \\
\hline $18454-0158$ & $184801.3 \pm 1.0$ & $-015434 \pm 15$ & $1665 \mathrm{~L}$ & 39.6 & 30.0 & 44.0 & 0.35 & 0.10 & & \\
\hline $18463+0052$ & $184846.8 \pm 2.2$ & $005655 \pm 24$ & 1612R & 67.32 & 67.0 & 70.0 & 2.26 & 0.09 & & $\mathrm{OH} / \mathrm{IR}$ \\
\hline $18488+0000$ & $185130.5 \pm 1.0$ & $000321 \pm 16$ & $1665 \mathrm{R}$ & 79.57 & 79.0 & 87.0 & 4.52 & 0.07 & 6 & \\
\hline & & & $1667 \mathrm{R}$ & 77.90 & & & 2.66 & 0.05 & & \\
\hline $18507+0121$ & $185318.2 \pm 1.2$ & $012430 \pm 18$ & $1665 \mathrm{~L}$ & 55.78 & 53.0 & 56.0 & 2.00 & 0.08 & 14 & \\
\hline & & & $1667 \mathrm{~L}$ & 53.88 & & & 1.10 & 0.09 & & \\
\hline $18527+0301$ & $185446.5 \pm 2.5$ & $030507 \pm 40$ & $1665 \mathrm{R}$ & 74.44 & 72.0 & 75.0 & 0.21 & 0.02 & 48 & \\
\hline & & & $1667 \mathrm{~L}$ & 73.30 & & & 0.16 & 0.02 & & \\
\hline $18553+0414$ & $185750.7 \pm 1.2$ & $041836 \pm 18$ & $1720 \mathrm{~L}$ & 6.63 & 4.0 & 8.0 & 0.67 & 0.03 & & \\
\hline $18566+0408$ & $185908.7 \pm 1.1$ & $041021 \pm 17$ & $1665 \mathrm{~L}$ & 83.41 & 52.0 & 92.0 & 1.00 & 0.12 & 7 & \\
\hline & $185910.4 \pm 1.0$ & $041321 \pm 14$ & $1667 \mathrm{~L}$ & 81.52 & & & 0.50 & 0.14 & & \\
\hline $19035+0641$ & $190601.6 \pm 0.0$ & $064635 \pm 01$ & $1665 \mathrm{~L}$ & 32.44 & 24.2 & 36.2 & 90.00 & 0.61 & 0.2 & 1 \\
\hline & & & $1667 \mathrm{R}$ & 27.33 & & & 22.30 & 0.27 & & \\
\hline $19092+0841$ & $191145.9 \pm 0.4$ & $084649 \pm 06$ & $1665 \mathrm{R}$ & 57.87 & 54.0 & 62.0 & 3.45 & 0.04 & 3 & \\
\hline & $191146.6 \pm 0.4$ & $084619 \pm 10$ & $1667 \mathrm{~L}$ & 60.51 & & & 1.75 & 0.04 & & \\
\hline $19118+0945$ & $191429.7 \pm 1.6$ & $095147 \pm 46$ & $1665 \mathrm{R}$ & 61.25 & 61.0 & 71.0 & 0.35 & 0.05 & & \\
\hline & & & $1667 \mathrm{~L}$ & 58.90 & & & 0.22 & 0.05 & & \\
\hline $19217+1651$ & $192357.9 \pm 0.9$ & $165642 \pm 13$ & $1665 \mathrm{~L}$ & 0.21 & -2.0 & 10.0 & 1.35 & 0.05 & 1 & \\
\hline & & & $1667 \mathrm{~L}$ & 6.69 & & & 1.22 & 0.05 & & \\
\hline $19220+1432$ & $192419.7 \pm 1.5$ & $143723 \pm 30$ & $1720 \mathrm{R}$ & 60.67 & 59.5 & 61.0 & 0.44 & 0.08 & & \\
\hline
\end{tabular}


Table 2. continued.

\begin{tabular}{|c|c|c|c|c|c|c|c|c|c|c|}
\hline \multirow[t]{2}{*}{ IRAS Name } & \multicolumn{2}{|c|}{ Position } & \multirow[t]{2}{*}{ Frequency } & \multicolumn{3}{|c|}{ Velocity at } & \multirow{2}{*}{$\begin{array}{c}\text { Flux at } \\
S_{\text {peak }} \\
\text { Jy }\end{array}$} & \multirow{2}{*}{$\begin{array}{c}\mathrm{rms} \\
\mathrm{Jy}\end{array}$} & \multirow[t]{2}{*}{$\bar{R}$} & \multirow[t]{2}{*}{ Ref./Notes } \\
\hline & $\begin{array}{c}\text { RA(J2000) } \\
\text { h m s }\end{array}$ & $\begin{array}{c}\operatorname{Dec}(\mathrm{J} 2000) \\
\circ,, \prime\end{array}$ & & $V_{\text {peak }}$ & $\frac{V_{\min }}{\mathrm{km} \mathrm{s}^{-1}}$ & $V_{\max }$ & & & & \\
\hline \multirow{3}{*}{$19374+2352$} & $193937.4 \pm 4.0$ & $235953 \pm 109$ & $1665 \mathrm{R}$ & 37.06 & 35.0 & 40.0 & 0.46 & 0.06 & & \\
\hline & & & $1667 \mathrm{R}$ & 37.00 & & & 0.13 & 0.02 & & \\
\hline & & & $1720 \mathrm{R}$ & 37.05 & & & 0.17 & 0.03 & & \\
\hline $19388+2357$ & $194110.2 \pm 2.2$ & $240344 \pm 25$ & $1665 \mathrm{~L}$ & 35.81 & 34.0 & 39.0 & 0.32 & 0.04 & 77 & \\
\hline $19410+2336$ & $194312.2 \pm 1.1$ & $234403 \pm 12$ & $1665 \mathrm{~L}$ & 20.67 & 20.0 & 23.0 & 0.71 & 0.03 & 48 & \\
\hline $20062+3550$ & $200812.7 \pm 1.2$ & $355920 \pm 20$ & $1665 R$ & 0.77 & -1.0 & 2.2 & 0.17 & 0.03 & 59 & \\
\hline $20126+4104$ & $201426.06 \pm 0.002$ & $411332.63 \pm 0.02$ & $1665 \mathrm{R}$ & -12.27 & -16.0 & 2.0 & 2.37 & 0.11 & 16 & 2 \\
\hline $20188+3928$ & $202041.8 \pm 1.0$ & $393742 \pm 12$ & $1720 \mathrm{~L}$ & -1.03 & -3.0 & 4.0 & 4.18 & 0.14 & & \\
\hline $20227+4154$ & $202434.6 \pm 1.3$ & $420612 \pm 22$ & $1665 \mathrm{~L}$ & 24.29 & 10.0 & 25.0 & 0.80 & 0.07 & & \\
\hline \multirow[t]{2}{*}{$22198+6336$} & $222100.7 \pm 1.3$ & $635157 \pm 16$ & $1665 \mathrm{~L}$ & -12.14 & -23.0 & -10.0 & 2.00 & 0.08 & & \\
\hline & $222137.6 \pm 0.4$ & $635147 \pm 08$ & $1667 \mathrm{~L}$ & -13.00 & & & 3.50 & 0.17 & & \\
\hline \multirow[t]{2}{*}{$22272+6358$} & $222858.5 \pm 0.8$ & $641552 \pm 14$ & $1665 \mathrm{~L}$ & -12.12 & -12.0 & -8.0 & 1.10 & 0.06 & 83 & \\
\hline & & & $1667 \mathrm{~L}$ & -11.96 & & & 1.68 & 0.07 & & \\
\hline $23139+5939$ & $231606.8 \pm 1.0$ & $595852 \pm 13$ & $1612 \mathrm{R}$ & -72.93 & -74.0 & -68.0 & 0.63 & 0.11 & & \\
\hline \multicolumn{11}{|c|}{ Masers sources offset } \\
\hline \multicolumn{2}{|c|}{$04579+4703$} & & $1720 \mathrm{R}$ & -17.70 & -18.1 & -16.8 & 0.22 & 0.05 & & \\
\hline \multicolumn{2}{|l|}{$06382+0939$} & & $1665 \mathrm{R}$ & 11.48 & 9.2 & 13.0 & 0.80 & 0.16 & & \\
\hline \multicolumn{2}{|l|}{$18408-0348$} & & $1665 \mathrm{R}$ & 89.60 & 88.0 & 113.0 & 1.00 & 0.10 & & \\
\hline \multicolumn{2}{|l|}{$18511+0146$} & & $1665 \mathrm{R}$ & 47.91 & 47.0 & 49.0 & 0.38 & 0.04 & & \\
\hline \multirow{2}{*}{\multicolumn{2}{|c|}{$18540+0220$}} & & $1665 \mathrm{R}$ & 49.13 & 48.0 & 53.5 & 53.73 & 0.19 & & \\
\hline & & & $1667 \mathrm{~L}$ & 50.38 & & & 10.57 & 0.09 & & \\
\hline \multirow[t]{3}{*}{$18586+0106$} & & & $1665 \mathrm{~L}$ & 42.00 & 38.5 & 46.0 & 0.50 & 0.18 & & \\
\hline & & & $1667 \mathrm{~L}$ & 42.50 & & & 0.70 & 0.17 & & \\
\hline & & & $1720 \mathrm{R}$ & 38.81 & & & 3.00 & 0.31 & & \\
\hline \multicolumn{2}{|l|}{$20099+3640$} & & $1665 \mathrm{R}$ & -42.74 & -43.0 & -42.0 & 0.30 & 0.04 & & \\
\hline \multicolumn{11}{|c|}{$\overline{O H / I R}$ sources offset } \\
\hline \multicolumn{2}{|c|}{$18258-0737$} & & $1612 \mathrm{R}$ & 93.00 & 63.0 & 95.0 & 2.00 & 0.10 & & $\mathrm{OH} / \mathrm{IR}$ \\
\hline \multicolumn{2}{|l|}{$18348-0616$} & & $1612 \mathrm{~L}$ & 70.00 & 25.0 & 73.0 & 1.50 & 0.18 & & $\mathrm{OH} / \mathrm{IR}$ \\
\hline \multicolumn{2}{|l|}{$18424-0329$} & & $1612 \mathrm{~L}$ & 40.00 & 38.0 & 82.0 & 0.50 & 0.13 & & $\mathrm{OH} / \mathrm{IR}$ \\
\hline \multicolumn{2}{|l|}{$18565+0349$} & & $1612 \mathrm{~L}$ & 27.00 & 25.0 & 62.0 & 1.50 & 0.11 & & $\mathrm{OH} / \mathrm{IR}$ \\
\hline
\end{tabular}

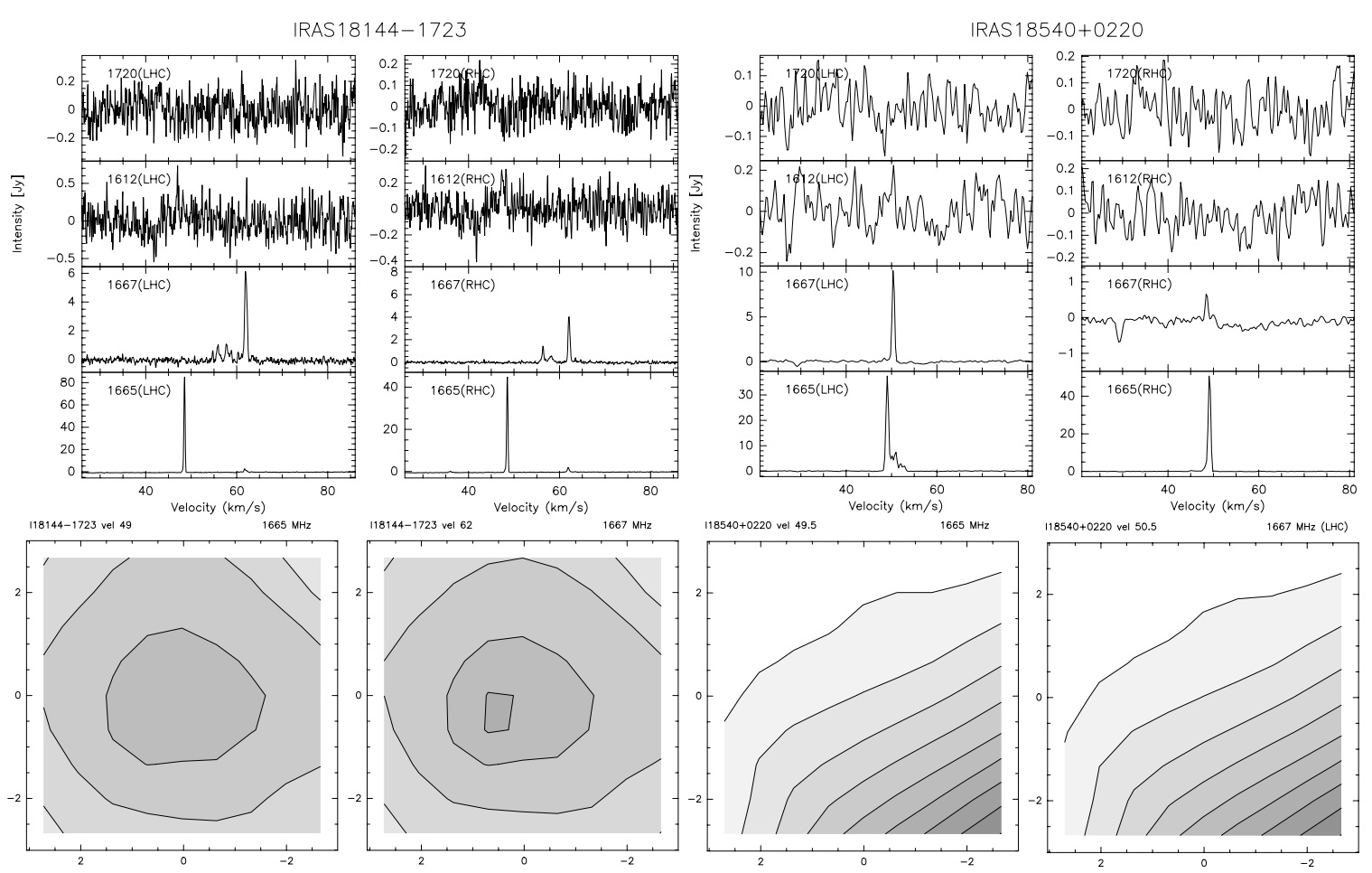

Fig. 2. Examples spectra and maps of $\mathrm{OH}$ masers detected with the GBT. The maps shows if the $\mathrm{OH}$ maser emission is consistent with the IRAS position (as for IRAS 18144-1723, left panels) or offset from it (as for IRAS 18540+0220, right panels). The maps are 9-points maps of the integrated intensities over the velocity range of each maser component. The contours range from $10 \%$ to $90 \%$ of the peak flux given in Table 2 in steps of $10 \%$. The axes show RA and Dec offset from the IRAS position in arcminutes. 
Table 3. Non-OH maser sources cataloged as: sources with thermal emission and/or absorption in one or more of the $\mathrm{OH}$ maser lines, sources with no detectable features, not confirmed maser sources (sources detected with Nançay only), not confirmed OH/IR sources.

\begin{tabular}{lccc}
\hline \hline IRAS sources & with thermal absorption and/or & thermal emission \\
$00420+5530$ & $18167-1614$ & $18551+0302$ & $20081+2720$ \\
$05168+3634$ & $18172-1548$ & $18567+0700$ & $20180+3558$ \\
$05345+3157$ & $18212-1320$ & $18571+0326$ & $20205+3948$ \\
$05373+2349$ & $18223-1243$ & $18571+0349$ & $20216+4107$ \\
$06061+2151$ & $18256-0742$ & $18596+0536$ & $20217+3947$ \\
$06063+2040$ & $18288-0158$ & $19001+0402$ & $20220+3728$ \\
$06105+1756$ & $18306-0835$ & $19002+0454$ & $20293+3952$ \\
$06299+1011$ & $18337-0743$ & $19012+0505$ & $20293+4007$ \\
$06303+1021$ & $18363-0554$ & $19012+0536$ & $20319+3958$ \\
$17417-2851$ & $18396-0431$ & $19023+0538$ & $20332+4124$ \\
$17450-2742$ & $18426-0204$ & $19045+0518$ & $20333+4102$ \\
$17495-2624$ & $18437-0216$ & $19088+0902$ & $20343+4129$ \\
$17582-2234$ & $18445-0222$ & $19175+1357$ & $21078+5211$ \\
$18024-2231$ & $18447-0229$ & $19198+1423$ & $21548+5747$ \\
$18039-2052$ & $18454-0136$ & $19295+1637$ & $22551+6221$ \\
$18134-1942$ & $18517+0437$ & $19332+2028$ & $23026+5948$ \\
$18156-1343$ & $18530+0215$ & $19343+2026$ & $23033+5951$ \\
$18159-1550$ & $18532+0047$ & $19368+2239$ & $23140+6121$ \\
$18159-1648$ & $18537+0145$ & $20050+2720$ & $23545+6508$ \\
$18162-1612$ & $18544+0112$ & $20051+3435$ & \\
\hline IRAS sources with no detected & absorption or emission \\
$00117+6412$ & $18272-1217$ & $19542+3004$ & $22187+5559$ \\
$01420+6401$ & $18317-0513$ & $20028+2903$ & $22267+6244$ \\
$03211+5446$ & $18355-0550$ & $20056+3350$ & $22305+5803$ \\
$04034+5116$ & $18372-0541$ & $20106+3545$ & $22344+5909$ \\
$05490+2658$ & $18470-0044$ & $20278+3521$ & $22457+5751$ \\
$05553+1631$ & $18472-0022$ & $20286+4105$ & $22506+5944$ \\
$06068+2030$ & $18521+0134$ & $20321+4112$ & $22570+5912$ \\
$06103+1523$ & $19043+0726$ & $20406+4555$ & $23146+5954$ \\
$06104+1524$ & $19077+0839$ & $20444+4629$ & $23151+5912$ \\
$06155+2319$ & $19094+0944$ & $21046+5110$ & $23152+6034$ \\
$06291+0421$ & $19183+1556$ & $21080+4758$ & $23314+6033$ \\
$06308+0402$ & $19045+0813$ & $21202+5157$ & $23330+6437$ \\
$06584-0852$ & $19213+1723$ & $21307+5049$ & $23385+6053$ \\
$17571-2328$ & $19266+1745$ & $21336+5333$ & $23448+6010$ \\
$17504-2519$ & $19282+1814$ & $21391+5802$ & $23507+6230$ \\
$18014-2428$ & $19403+2258$ & $21519+5613$ & \\
$18123-1203$ & $19411+2306$ & $21526+5728$ & \\
$18151-1208$ & $19413+2332$ & $22134+5834$ & \\
$18197-1351$ & $19458+2442$ & $22147+5948$ & \\
$18247-1147$ & $19471+2641$ & $22172+5549$ & \\
\hline Maser sources not confirmed & & \\
$00070+6503$ & $18311-0701$ & $18431-0312$ & $19074+0752$ \\
\hline OH/IR sources not confirmed & & \\
$18308-0841$ & $18460-0307$ & & \\
\hline & & & \\
\hline
\end{tabular}

sources. A brief description and discussion of each of the $\mathrm{OH}$ maser sources is given in Appendix A.

\section{Detection statistics and analysis}

The survey observations detected $63 \mathrm{OH}$ maser sources out of 217 IRAS sources. For 46 of these the association with the IRAS source is confirmed by maps made with the GBT. Of these 36 are new detections. That gives a detection rate of $29 \%$ (including unconfirmed and not associated with IRAS position sources) or $21 \%$ if only the confirmed IRAS associated sources are considered. This is very similar to the $22 \%$ detection rate of Cohen et al. (1988) although the Cohen et al. sample contained only sources with $F_{60}>1000 \mathrm{Jy}$. Cohen et al. also found that higher $F_{60}$ was correlated with a higher probability of the presence of $\mathrm{OH}$ masers. There is little evidence for such a correlation in the objects observed in this survey, although the small number of high flux sources in this sample makes it difficult draw any firm conclusion.

Figure 3 summarises the detection statistics for $\mathrm{OH}, \mathrm{H}_{2} \mathrm{O}$ and $6.7 \mathrm{GHz}$, Class II, $\mathrm{CH}_{3} \mathrm{OH}$ masers towards the sample. $\mathrm{OH}$ and $\mathrm{CH}_{3} \mathrm{OH}$ masers show very similar percentages of detections. Indeed, among the detected $\mathrm{OH}$ masers sources, the two types of maser have $67 \%$ of sources in common. The number of detections of the three maser types towards the sub-samples is also shown. The S02 and High samples show similarly higher detection rates than the Low sample for which $70 \%$ of its sources have no maser emission. Not only are masers of any type relatively rare towards the Low sub-sample, sources with all three types of maser are particularly uncommon. In the Low sub-sample there are only three such sources, namely IRAS $19092+0841$, IRAS 18024-2119 and IRAS 18144-1723. Water masers show the highest detection rates towards the High sample (46\%), while $\mathrm{CH}_{3} \mathrm{OH}$ masers show high detection rate towards $\mathrm{S} 02$ sample (42\%). Perhaps surprisingly there is a significant difference in the detection rates of $\mathrm{SO} 2$ and High sample for $\mathrm{CH}_{3} \mathrm{OH}$ maser, $42 \%$ and $25 \%$ respectively. $\mathrm{OH}$ masers have similar detection rates towards the High and S02 samples, $26 \%$. The sources which show only $\mathrm{OH}$ maser emission are mostly from the Low sample. This point is discussed in Sect. 6.3.

\section{1. $\mathrm{OH}$ maser flux densities}

Figure 4 shows the distribution of $1665 \mathrm{MHz}$ peak flux densities towards the three sub-samples, High sample (solid line), Low sample (dashed line) and S02 sample (dotted line). The right panel of Fig. 4 shows an expanded view for sources with $1665 \mathrm{MHz}$ flux density weaker than 4.5 Jy. These figures show that nearly half of the detected sources have peak flux densities $\lesssim 1 \mathrm{Jy}$. The significance of this result is discussed in Sect. 6.2. Figure 4 also shows a further difference between the different sub-samples. Most of the Low sample have $1665 \mathrm{MHz}$ flux densities between 0.25 and $0.75 \mathrm{Jy}$ while most of those of the S02 sample between 0.75 and $1.25 \mathrm{Jy}$ and the High sample shows a wider spread of $1665 \mathrm{MHz}$ flux densities.

\subsection{IRAS flux densities and colour analysis}

The correlation of maser emission and IR flux has been studied by many authors to examine if masers are pumped by IR photons or not. A correlation of $\mathrm{OH}$ maser flux and IR flux has been clearly seen at 60 and $100 \mu \mathrm{m}$ by several authors (Cohen et al. 1988; Moore et al. 1988; Slysh et al. 1997). Figure 5 plots the $1665 \mathrm{MHz}$ peak flux densities against the IRAS flux densities at $12,25,60$ and $100 \mu \mathrm{m}$ for the sample observed here. The top-left panel of $F_{60}$ vs. $F_{1665}$ also shows the results of previous studies: the Slysh et al. (1997) data upper limit (dashed line) corresponds to $F_{\mathrm{OH}}=0.024 F_{60}$ and the dotted line is the upper limit line of Moore et al. (1988) data (corresponding to $\left.F_{\mathrm{OH}}=0.1 F_{60}\right)$. The solid line corresponds to equal flux densities. Although the sources searched here have flux densities similar to Slysh et al. (1997), the survey results are more consistent with the Moore et al. (1988) line and appear to be consistent with an extension of Moore et al. sample to lower IR flux densities. The distribution at 60 and $100 \mu \mathrm{m}$ flux densities confirm previous conclusions that, at these wavelengths, a minimum IR flux density is required for a given maser line strength (Cohen et al. 1988; Moore et al. 1988; Slysh et al. 1997). 


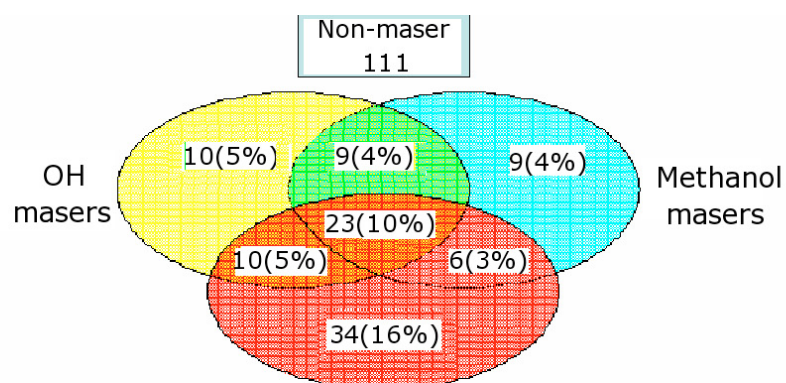

Water masers

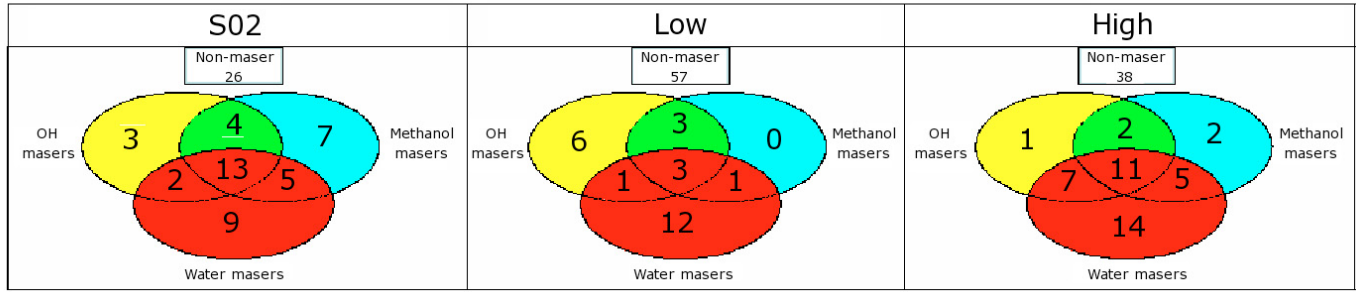

Fig. 3. Upper panel: number (and percentage of sample) of detected sources of the 217 HMPOs sample based on which masers are present. Note that the 7 offset star forming region candidates in Table 2 are considered here while the $5 \mathrm{OH} / \mathrm{IR}$ candidates detected are excluded. More than half of the sample do not show maser emission. Bottom panels: same as upper panel but divided by subsamples. For the whole sample there are 100 sources associated one or more types of maser, while there are 43, 26 and 42 maser sources in the S02, Low and High subsamples respectively. Note that some sources are common in the S02 and High subsamples.
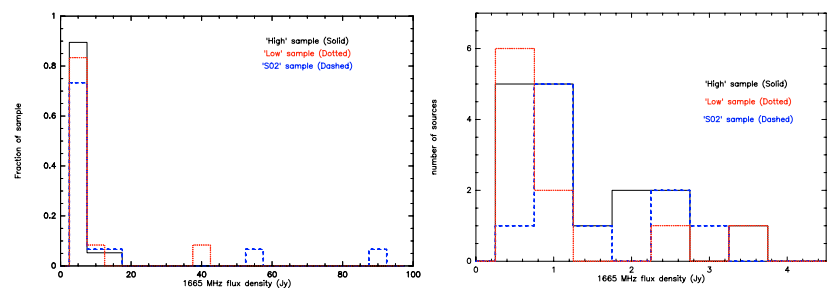

Fig. 4. The flux densities at $1665 \mathrm{MHz}$ line towards the three subsamples, high sample (solid line), low sample (dashed line) and S02 sample (dotted line). An expanded view for the $1665 \mathrm{MHz}$ flux densities weaker than $4.5 \mathrm{Jy}$ is re-plotted in the righthand panel.

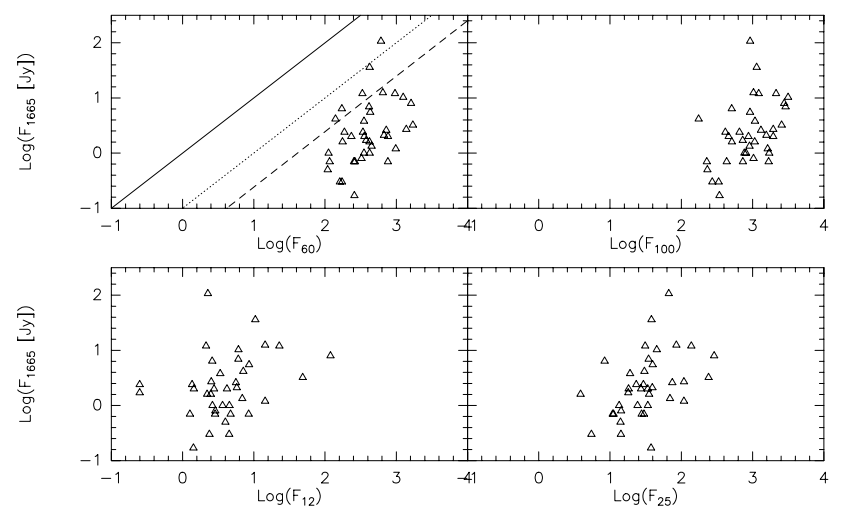

Fig. 5. OH $1665 \mathrm{MHz}$ peak flux densities of the detected masers are plotted against IRAS flux densities at 12, 25, 60 and $100 \mu \mathrm{m}$. The solid line, in the top-left panel, corresponds to equal flux densities, the dashed line is the line of Slysh et al. (1997) data (corresponding to $F_{\mathrm{OH}}=$ $\left.0.024 F_{60}\right)$ and the dotted line is the line of Moore et al. (1988) data (corresponding to $F_{\mathrm{OH}}=0.1 F_{60}$ ).

Figure 6 plots the [100-60] colour index vs. OH $1665 \mathrm{MHz}$ maser luminosity, $L_{1665}$. There may be a suggestion of a lower envelope to the distribution at $\log L_{1665} \sim 0.7$ [100-60]

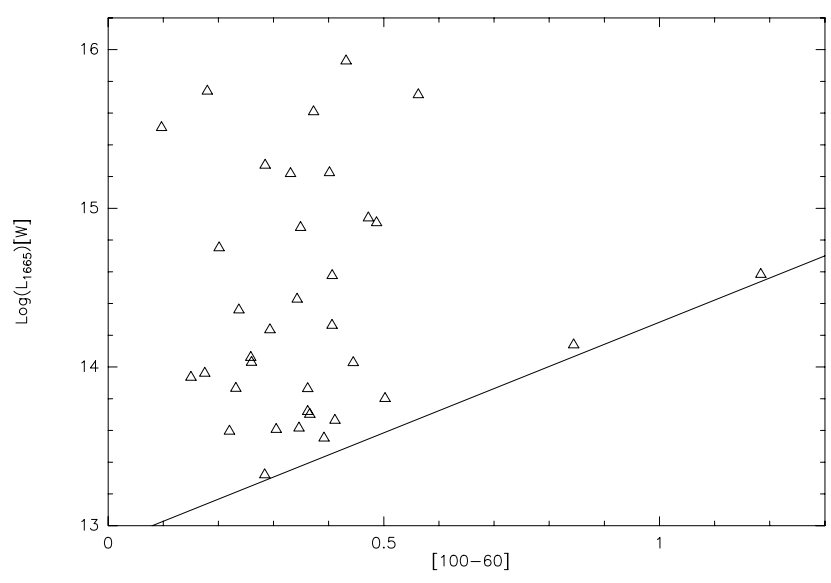

Fig. 6. The $1665 \mathrm{MHz} \mathrm{OH}$ luminosities of the detected masers are plotted against the [100-60] colour index. There may be a suggestion of a lower envelope to the distribution at about $\log L_{1665} \sim 0.7 \times[100-60]$ (solid line). Three sources with suspect distances (IRAS 18024-2119, $20062+3550$ and $20227+4154$ ) are not shown on the plot.

indicating masers are found prefentially towards sources with warmer radiation temperatures.

To see if there is a difference in IR flux densities or colours between maser and non-maser sources, IRAS sources associated with one or more type of maser were compared with the rest of the sources. Table 4 gives the results of Kolmogorov-Smirnov (KS) test for the probability $D$, that the flux density or colour distribution between maser and non-maser source are different. The table shows that in general, the maser sources do show different flux densities and colours from non-maser ones. The difference between maser sources and non-maser sources is most evident in the [60-12] and [100-12] colours for all types of masers where $D \equiv 100 \%$ and in the [25-12] colour for $\mathrm{H}_{2} \mathrm{O}$ and $\mathrm{CH}_{3} \mathrm{OH}$ masers where $D \geq 99.9 \%$. Figure 7 plots the distribution of these three colour indices for the $\mathrm{OH}$ maser and nonmaser sources to demonstrate the last points. It can also be seen 
Table 4. Results of KS test for the maser and non-maser population being drawn from the same populations. The table shows the probability $D$ that the maser and non-maser sources are drawn from different populations. Clearly the most consistent difference is in [60-12] and [100-12] for which maser sources of all types have consistently different colours than the non-maser sources. Values greater than $99.9 \%$ shown as $100 \%$. For comparison a $3 \sigma$ result would correspond to $D=99.87 \%$.

\begin{tabular}{cccccccccc}
\hline \hline & {$[25-12]$} & {$[60-12]$} & {$[60-25]$} & {$[100-60]$} & {$[100-12]$} & $F_{12}$ & $F_{25}$ & $F_{60}$ & $F_{100}$ \\
Maser type/s & $\%$ & $\%$ & $\%$ & $\%$ & $\%$ & $\%$ & $\%$ & $\%$ & $\%$ \\
\hline $\mathrm{OH}$ & 96.5 & 100 & 53.6 & 19.7 & 100 & 98.8 & 84.8 & 92.4 & 96.5 \\
$\mathrm{H}_{2} \mathrm{O}$ & 100 & 100 & 61.3 & 12.4 & 100 & 83.1 & 99.2 & 99.7 & 99.5 \\
$\mathrm{CH}_{3} \mathrm{OH}$ & 99.9 & 100 & 91.7 & 98 & 100 & 100 & 41.1 & 99.6 & 100 \\
$\mathrm{OH}, \mathrm{H}_{2} \mathrm{O} \& \mathrm{CH}_{3} \mathrm{OH}$ & 96.5 & 100 & 81.5 & 74.5 & 100 & 99.9 & 81.5 & 99.4 & 99.4 \\
\hline
\end{tabular}
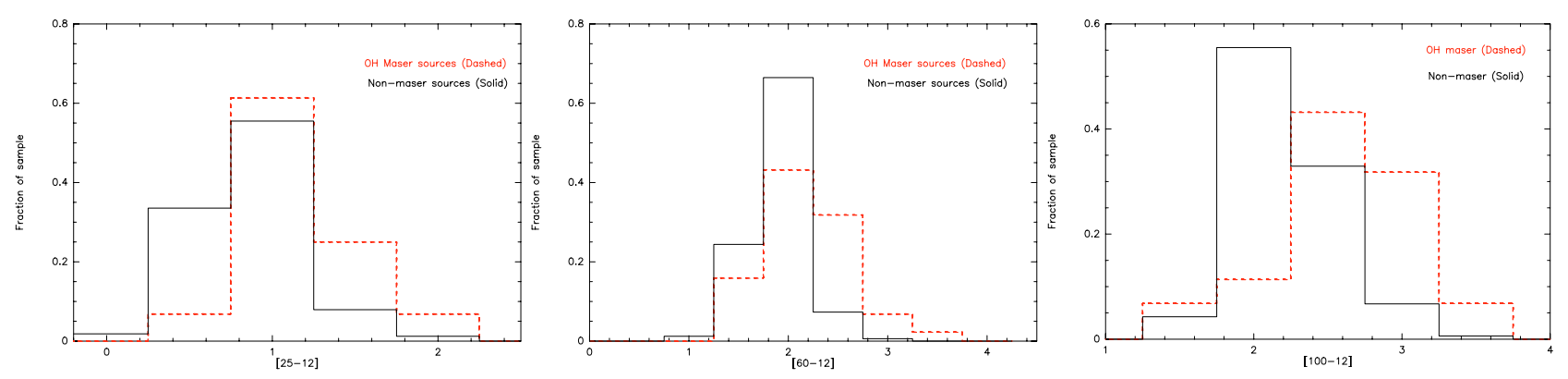

Fig. 7. The [25-12] (left panel), [60-12] (middle panel) and [100-12] (right panel) colour indices for OH maser sources (dashed lines) and non-OH-maser sources (solid lines). In each case $\mathrm{OH}$ maser sources have on average redder colours than the non-maser sources and objects with colours $[25-12]>1.2,[60-12]>2.2$ and $[100-12]>2.7$ are dominated by sources with $\mathrm{OH}$ masers.

in Fig. 1, where it is clear that sources with $\mathrm{OH}$ masers dominate over non-maser sources in the regions [25-12] $>1.2$ and $[60-12]>2.2$. Even for colours $\sim 0.2$ smaller than these values, the maser sources make up $\sim 50 \%$ of the population, a higher fractor than for sources with smaller colours and for the sample overall. The redder colours of the maser sources is unlikely to be affected by the uncertainties in the colours of the sources unless the uncertainties preferentially affect maser sources and non-maser sources in different senses.

The [25-12] and [60-12] colour indices are the ones used by WC89 to identify UCHII regions. These results show that maser sources tend to be relatively faint at $12 \mu \mathrm{m}$ compared to their fluxes at longer wavelengths. Figure 8 shows the four IRAS flux densities against each other for maser sources (squares) and nonmaser sources (dots). The difference between maser sources and non-maser sources is obvious in the lower panels which include the $12 \mu \mathrm{m}$ flux densities. On the other hand, as Table 4 also shows, sources with only $\mathrm{OH}$ or $\mathrm{H}_{2} \mathrm{O}$ masers have [60-25] and [100-60] colours indistinguishable from non-maser sources.

Given the differences in [60-12] and [100-12] colours of sources with any type of maser, it is interesting to ask whether there is any systematic difference in source colour as a function of maser type. Figure 9 compares these colours for sources with each type of maser showing that there is no significant difference in the distribution of these colours as a function of maser type. For this sample there appears to be no evidence in their IRAS colours that different types of maser trace sources in significantly different evolutionary states.

Regarding the 100, 60, 25 and $12 \mu \mathrm{m}$ flux densities, the probabilities, $D$ in Table 4 are relatively large for all flux densities, except the $\mathrm{CH}_{3} \mathrm{OH}$ only sources at $25 \mu \mathrm{m}$. However only for few maser-flux combinations are the individual $D$ probabilities statistically significant at a $3 \sigma$ level. Although methanol masers are believed to be excited by mid-IR photons (Cragg et al. 2005), Fig. 10 shows that there is no clear correlation between $\mathrm{CH}_{3} \mathrm{OH}$ maser and $12 \mu \mathrm{m}$ flux densities.
Figure 11 compares the luminosity of maser sources and non-maser sources. The distributions are statistically indistinguishable, indicating that masers are not preferentially associated with only the more luminous sources.

Comparing the IRAS colour indices to the ratio of $\mathrm{CH}_{3} \mathrm{OH}$ and $\mathrm{OH}$ maser flux densities, Fig. 12 plots the ratio of the $\mathrm{OH}$ peak flux density to the $\mathrm{CH}_{3} \mathrm{OH}$ peak flux density, $R$, versus the IRAS colours of a source. According to Caswell (1998) the spread in the ratio $R$ reflects the range in evolutionary stage of the sources, but this figure does not show any evidence that $R$ depends on the colour of a source which might also be expected to evolve as a source evolves. In considering this result, it should be noted that the IRAS colours may suffer from confusion because of the poor spatial resolution of IRAS and maser observations combined with the tendency of massive stars to form in clusters (cf. Bourke et al. 2005).

\subsection{Velocity range of masers}

The range of velocities over which maser emission is observed can be used to investigate whether the emission from different masers arises from different material associated with the sources. Figure 13 shows the distribution of the range of velocity for each $\mathrm{OH}$ maser source detected (Table 2). For comparison the figure also shows the velocity range of the $\mathrm{H}_{2} \mathrm{O}$ and $\mathrm{CH}_{3} \mathrm{OH}$ masers from SHK2000, P91, S02 (and references therein) and Han et al. (1998). The righthand panel of Fig. 13 shows an expanded view of the sources with velocity range $\leq 20 \mathrm{~km} \mathrm{~s}^{-1}$.

For all three species the velocity range peaks at less than $10 \mathrm{~km} \mathrm{~s}^{-1}$. The $\mathrm{CH}_{3} \mathrm{OH}$ masers and $\mathrm{OH}$ masers extend up to velocity ranges of 17.5 and $22.5 \mathrm{~km} \mathrm{~s}^{-1}$ respectively. Overall the figures show that the $\mathrm{OH}$ and $\mathrm{CH}_{3} \mathrm{OH}$ agree very well in their distribution of velocity range, peaking at $\sim 5 \mathrm{~km} \mathrm{~s}^{-1}$, suggesting that these masers may originate in similar material around the sources. On the other hand the $\mathrm{H}_{2} \mathrm{O}$ masers show a quite different distribution. In some sources emission covering up to $65 \mathrm{~km} \mathrm{~s}^{-1}$ has been observed. However the distribution actually peaks at 

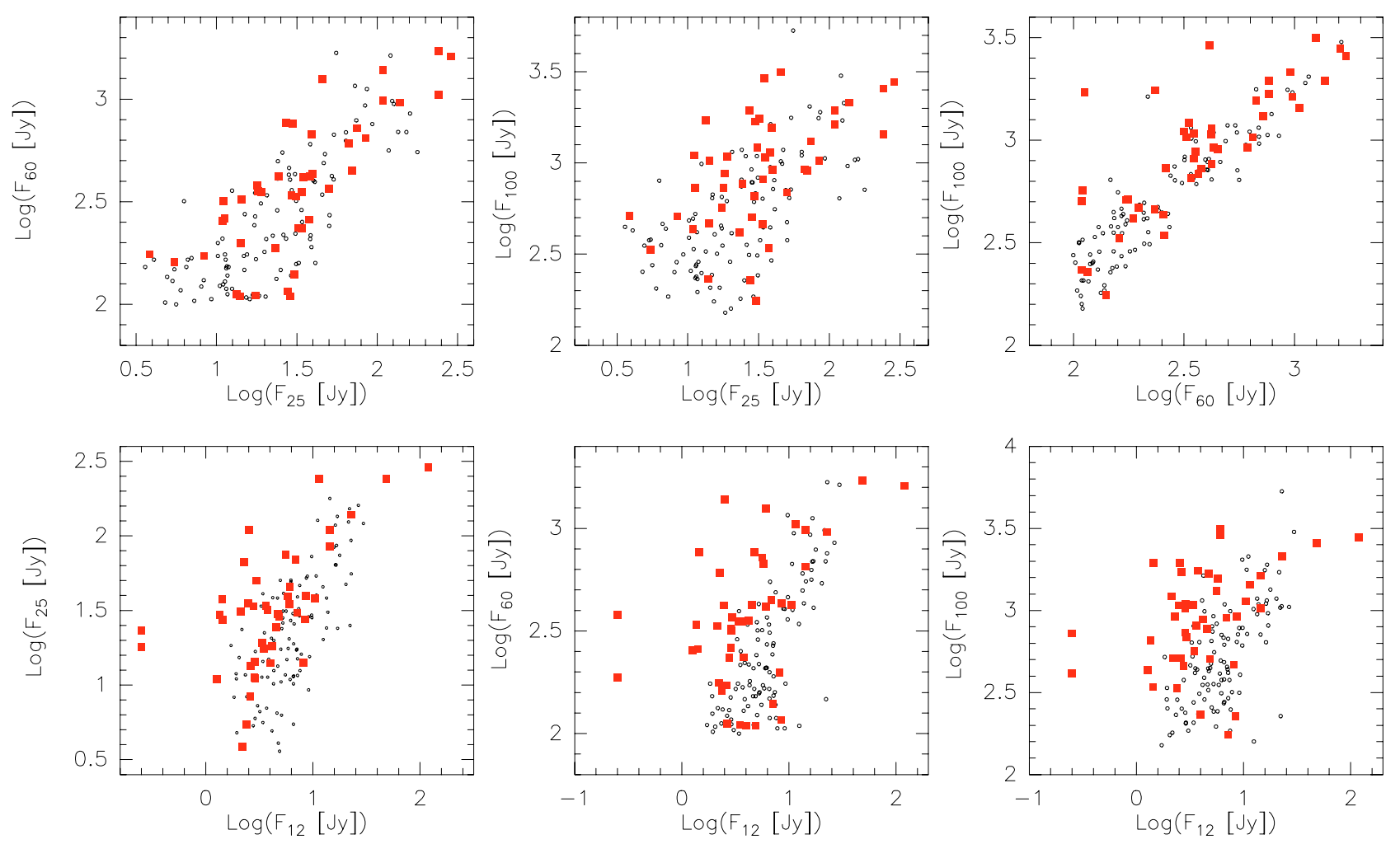

Fig. 8. Comparison of the IRAS flux densities for sources with $\mathrm{OH}$ masers (red squares) and those without OH masers (black dots). The difference between flux densities of maser source and non-maser ones is apparent in the lower panels where the effect of the $12 \mu \mathrm{m}$ is obvious, while there is no apparent difference in the upper panels.
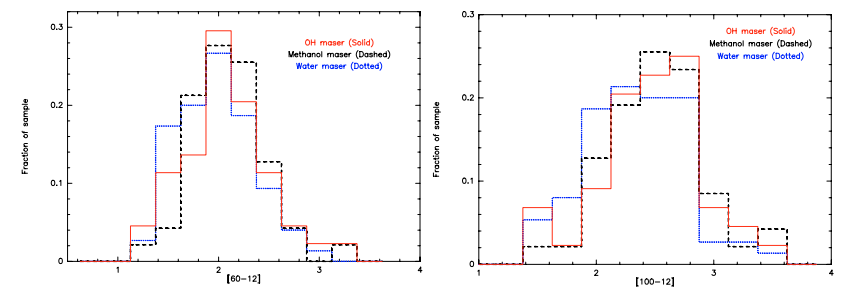

Fig. 9. [60-12] and [100-12] colours of sources with any type of maser, water (dotted lines), methanol (dashed lines) and $\mathrm{OH}$ (solid lines). There is no significant difference in the distribution of these colours as a function of maser type.

spreads $<4 \mathrm{~km} \mathrm{~s}^{-1}$, smaller than for the $\mathrm{OH}$ and $\mathrm{CH}_{3} \mathrm{OH}$. This suggests a two population structure, with one group of sources with velocity ranges between $\sim 1 \mathrm{~km} \mathrm{~s}^{-1}$ and $\sim 4-5 \mathrm{~km} \mathrm{~s}^{-1}$ and a second with velocity ranges $>5 \mathrm{~km} \mathrm{~s}^{-1}$.

The offset between the $\mathrm{OH}$ maser velocity and the velocity of the dense gas towards each source (M 96 and S02) is shown in Fig. 14 (left). Overall the $\mathrm{OH}$ maser velocities are distributed around the gas velocity, as they are also for some particular sources. On the other hand there are exceptions with some sources where the $\mathrm{OH}$ masers are offset by up to $\sim 20 \mathrm{~km} \mathrm{~s}^{-1}$ from the gas velocity. Comparing the velocity offset between different maser type and dense gas for sources with all three types of masers (Fig. 14, right), it is difficult to identify any global trend. However inspection of the observations shows 8 out of the 23 sources with all three masers have $\Delta V(\mathrm{OH})>\Delta V\left(\mathrm{CH}_{3} \mathrm{OH}\right)>$ $\Delta V\left(\mathrm{H}_{2} \mathrm{O}\right)$.

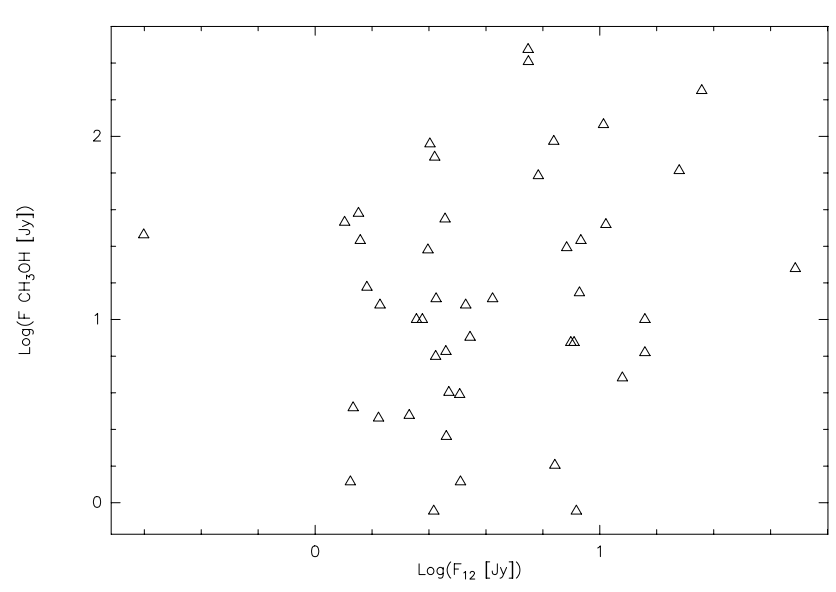

Fig. 10. Methanol maser peak flux densities are plotted against IRAS flux densities at $12 \mu$. No correlation is apparent.

\section{Discussion}

\subsection{Comparison of $\mathrm{OH}$ masers with $6668 \mathrm{MHz}$ methanol masers}

The similarity in detection rates and velocity ranges suggest an association between $\mathrm{OH}$ and Class II $\mathrm{CH}_{3} \mathrm{OH}$ masers in these sources as first suggested by Caswell et al. (1995) and modelled by Cragg et al. (2002). A similar result has also been found by Szymczak \& Gérard (2004) who searched a sample of $100 \mathrm{CH}_{3} \mathrm{OH}$ maser sources for $\mathrm{OH}$ masers. They found that $55 \%$ of $\mathrm{CH}_{3} \mathrm{OH}$ maser sources also have $\mathrm{OH}$ maser emission. Their results also show that $\mathrm{OH}$ and $\mathrm{CH}_{3} \mathrm{OH}$ masers cover similar velocity ranges. 


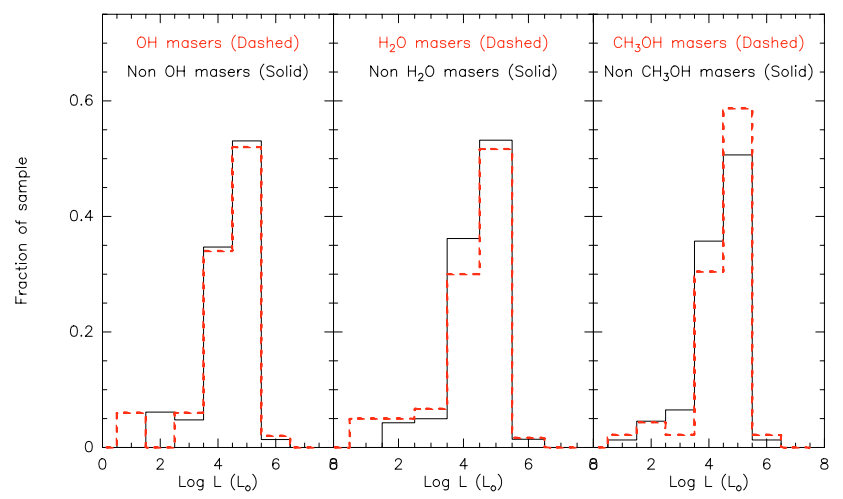

Fig. 11. Comparison of the luminosity of maser sources and non-maser sources. Note that the panels show sources with a specific maser type with sources are not associated with this type but may be associated with other types of masers.

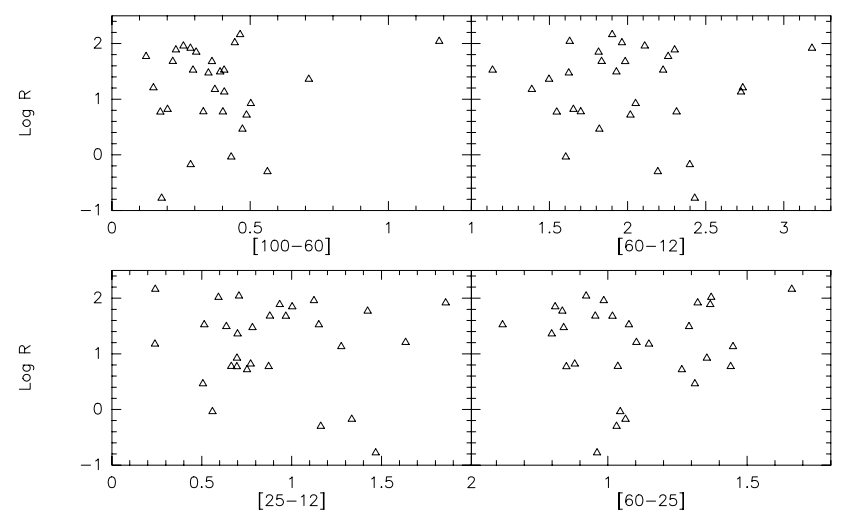

Fig. 12. Ratio of the $6668 \mathrm{MHz} \mathrm{CH}_{3} \mathrm{OH}$ peak flux density to the $1665 \mathrm{MHz} \mathrm{OH}$ peak flux density, $\log R$, versus IRAS colours.

We applied the suggestion of Caswell (1996) for characterizing the maser sites where methanol and $\mathrm{OH}$ masers occur using the ratio of peak methanol intensity to peak $1665-\mathrm{MHz}$ $\mathrm{OH}$ intensity. Figure 15 shows the distribution of the $\mathrm{OH}$ to $\mathrm{CH}_{3} \mathrm{OH}$ intensity ratio, $R=\frac{F(6668)}{F(1665)}$ (Table 2). For the 29 sources which show both $1665 \mathrm{MHz} \mathrm{OH}$ masers and $6668 \mathrm{MHz}$ masers, 12 sources have values of $R>32$ which places them in methanolfavoured region and 10 sources in $\mathrm{OH}$-favoured region $(R \leq 8)$.

There are an additional 12 sources which do not have $\mathrm{OH}$ maser emission but do have methanol masers and 20 sources with $\mathrm{OH}$ masers but no methanol masers. Limits on $R$ for these sources were obtained by adopting a three sigma limit on the presence of $\mathrm{OH}$ masers of $150 \mathrm{mJy}$ (typical of this survey) and a limit of $1500 \mathrm{mJy}$ for the presence of methanol masers (Pestalozzi et al. 2005). The resulting 3-sigma limits are also shown in Fig. 15. All the methanol non-detections are consistent with the sources being $\mathrm{OH}$ favoured. For the $\mathrm{OH}$ non-detections, one source with a lower limit on $R$ of 3 could be $\mathrm{OH}$ favoured, 8 sources are definitely methanol favoured and the remaining 3 have lower limits on $R$ between 8 and 32 .

The sources observed here have a very wide range in $R$, with measured values from ranging from 0.2 to 145 and three lower limits on $R$ in excess of 400 . The distribution of these values is in marked contrast to the results of Caswell (1996), who found most sources to have $R$ in the range 8 to 32, with a typical value of 16 . Over our whole sample, including the limits, 25 sources $(41 \%)$ are $\mathrm{OH}$-favoured. A similar fraction of the sample, 26 sources (43\%), are $\mathrm{CH}_{3} \mathrm{OH}$ favoured, which
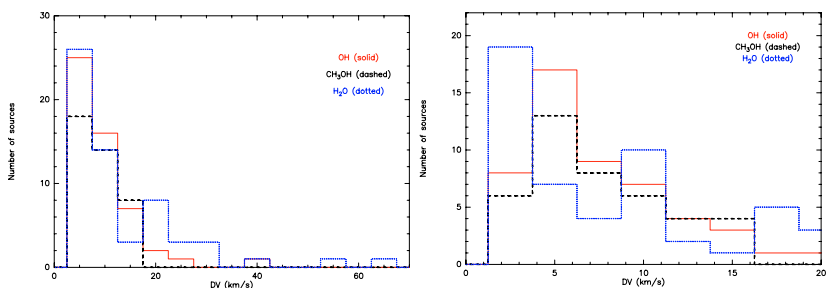

Fig. 13. The velocity spread of the masers associated with the whole sample: $\mathrm{OH}$ (solid line), $\mathrm{CH}_{3} \mathrm{OH}$ (dashed line) and $\mathrm{H}_{2} \mathrm{O}$ (dotted line). The righthand panel is an expanded view for velocities spreads $\leq 20 \mathrm{~km} \mathrm{~s}^{-1}$.

Caswell (1996) suggested are sources in an earlier evolutionary stage than $\mathrm{OH}$-favoured sources. Only 7 sources (11\%) have measured values of $R$ between 8 and 32 plus there are a further 3 lower limits (corresponding to a further $5 \%$ of the sample) which could place these sources in this range of $R$. If $R$ does indeed trace the evolutionary status of sources as Caswell has suggested, then these results suggest that the sample observed here represent a particular mix of sources in different evolutionary stages distinct from that in the sample observed by Caswell. The high fraction of methanol favoured sources here suggesting that this sample contains a higher portion of younger objects.

\section{2. $\mathrm{OH}$ maser flux densities}

Figure 4 shows that nearly half of the detected sources show $\mathrm{OH}$ flux densities $\lesssim 1 \mathrm{Jy}$, which indicates two points. First it demonstrates how less sensitive observations (with detection limits of $\gtrsim 1$ Jy, e.g. Cohen et al. 1988) could be missing a significant population of $\mathrm{OH}$ maser sources. More importantly it indicates that the sources observed here, which are believed to be in an early stage of formation with no observable UCHII regions, have lower $\mathrm{OH}$ flux densities than sources in more evolved objects associated with HII regions, where typical OH masers have flux densities $\geq 1 \mathrm{Jy}$.

Figure 6 shows that most of the $\mathrm{OH}$ maser sources prefer warmer radiation fields. This is probably because most of the FIR transitions which pump the $\mathrm{OH}$ maser are at wavelengths between $\sim 60 \mu \mathrm{m}$ and $100 \mu \mathrm{m}$ (Gray et al. 1992). The luminosity of the $1665 \mathrm{MHz}$ line spans a factor of about 300 across the sample, but the origin of this scatter is unclear. However it is likely that individual maser spots are saturated, so for a given source luminosity (especially in the spectral region $\sim 60 \mu \mathrm{m} \leq$ $\lambda \leq 100 \mu \mathrm{m}$ ) the maser luminosity may reflect the number of maser sites or volume of masing gas (Gray priv. comm.).

Models of $\mathrm{OH}$ maser emission show different physical conditions give rise to different combinations of $\mathrm{OH}$ maser lines. For example the Cragg et al. (2002) models show that $1665 \mathrm{MHz}$ masers trace a wider range of conditions that $1667 \mathrm{MHz}$ masers. In particular, gas kinetic temperatures $>75 \mathrm{~K}$, and gas number densities $\gtrsim 10^{7} \mathrm{~cm}^{-3}$ more strongly quench the emission at $1667 \mathrm{MHz}$ than the emission at $1665 \mathrm{MHz}$ suggesting that ratio of these lines fluxes may be a probe of the conditions in the gas, with masers seen in only the $1665 \mathrm{MHz}$ line tracing warmer, denser gas.

For the sample observed here 28 sources were detected in both the $1665 \mathrm{MHz}$ and $1667 \mathrm{MHz}$ lines. For all except four of these objects the $1665 \mathrm{MHz}$ line was stronger that the $1667 \mathrm{MHz}$ line. The ratio of the flux density at $1665 \mathrm{MHz}$ to that at $1667 \mathrm{MHz}$ for these objects is shown in Fig. 16. A further 16 objects were detected in only the $1665 \mathrm{MHz}$ line. The 


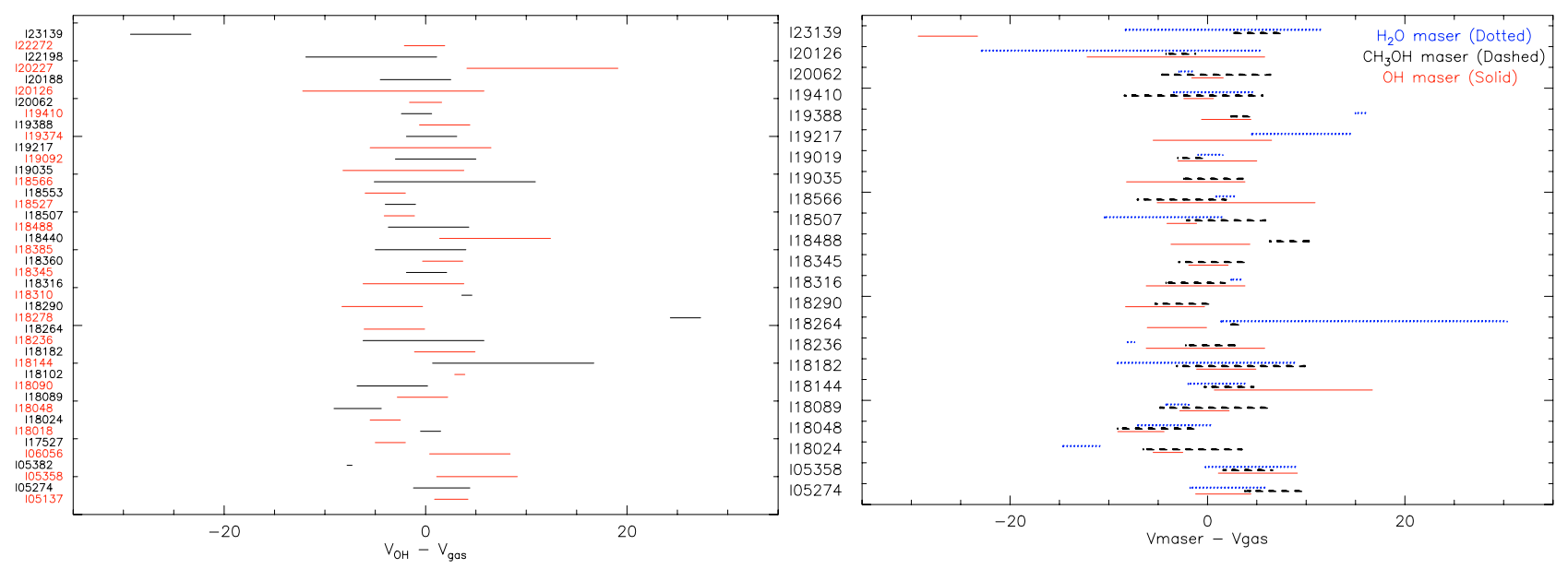

Fig. 14. Left hand panel: the velocity range relative to cloud gas velocity for $\mathrm{OH}$ maser sources. Right hand panel: the same as left hand panel but for the 23 sources showing three maser types. Note that some sources, although detected in $\mathrm{H}_{2} \mathrm{O}$ and/or $\mathrm{CH}_{3} \mathrm{OH}$, do not have published information on their velocity extent.

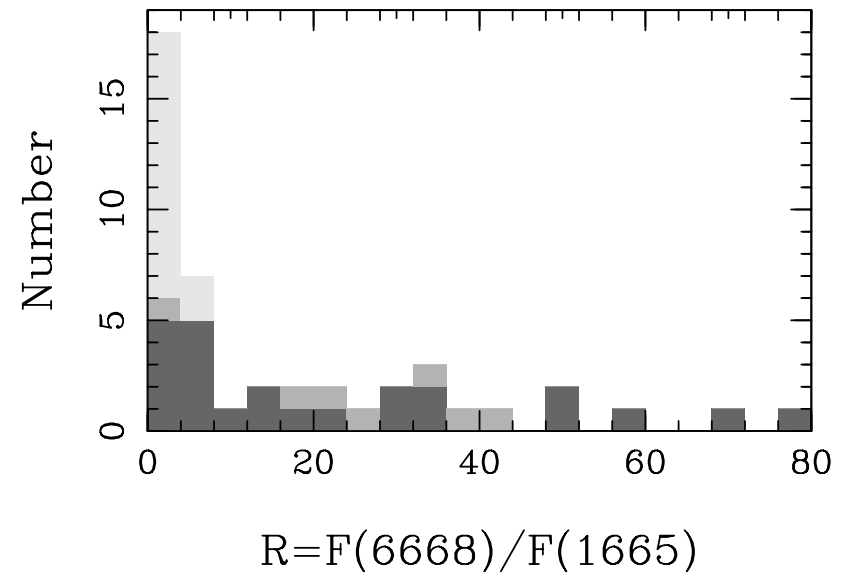

Fig. 15. Distribution of number of sources as a function of $R=\frac{F(6668)}{F(1665)}$, the ratio of the peak $\mathrm{CH}_{3} \mathrm{OH}$ maser intensity to the peak $\mathrm{OH}$ maser intensity. The darkest bars show measured values. The lightest bars show upper limits on $R$ for sources with no detected methanol masers and the mid-grey bars show the lower limits on $R$ for sources with no detected $\mathrm{OH}$ masers. There are 5 sources with measured values of $R>82$ and 5 lower limits with $R>82$ which are not shown on the plot. Caswell (1997) identified sources with $R \leq 8$ as $\mathrm{OH}$-favoured and those with $R \geq 32$ as methanol-favoured.

3-sigma lower limits on the $1665 \mathrm{MHz}$ to $1667 \mathrm{MHz}$ ratio for these sources are also shown the figure. The majority of sources have ratios of less $\sim 3$, but there is a tail of sources (and limits) up to ratios as large as $\sim 15$. Whether this range of intensity ratios reflects different physical conditions in these sources requires more detailed follow-up observations of the sources, particularly at higher angular resolution.

\section{3. $\mathrm{OH}$ maser only sources}

There is a group of newly detected sources associated with only $\mathrm{OH}$ maser emission; namely IRAS 06382+0939, 18408-0348, $18540+0220, \quad 18454-0158, \quad 18511+0146, \quad 18586+0106$, $19118+0945,19220+1432$ and $20099+3640$. The already known OH maser source IRAS 18018-2426 may also be in this group, although there has been no search for $\mathrm{CH}_{3} \mathrm{OH}$ maser emission towards it. However for only four of these objects,

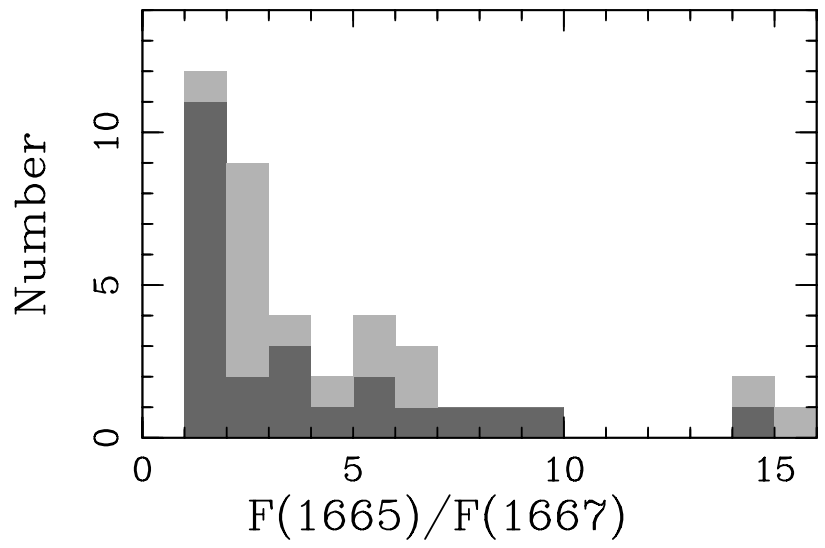

Fig. 16. The distribution of the ratio of peak $1665 \mathrm{MHz}$ flux density to peak $1667 \mathrm{MHz}$ flux density for the sources. The dark coloured bars show the measured ratios. There are four sources for which the $1667 \mathrm{MHz}$ line is stronger than the $1665 \mathrm{MHz}$ which are not shown. The light coloured bars show the 3 sigma lower limits for the sources which were detected at $1665 \mathrm{MHz}$ but not $1667 \mathrm{MHz}$. There is one source detected at $1667 \mathrm{MHz}$ but not $1665 \mathrm{MHz}$ which is also not shown. Sources where the masers are offset from the IRAS position are included in the distributions.

IRAS 18018-2426, 18454-0158, 19118+0945 and 19220+ 1432 are the $\mathrm{OH}$ masers positionally associated with the IRAS source. The $\mathrm{OH}$ masers towards the other sources are offset from the IRAS position and so it is possible that previous searches for other types of maser may have missed masers which are also offset from the IRAS position.

Interestingly, six of the ten $\mathrm{OH}$-only objects, including IRAS 18018-2426 and IRAS 19118+0945, are from the Low sub-sample of M 96 which contains sources which do not have UCHII region IRAS colours (WC89). However, four of these sources are associated with radio continuum emission and no equivalent observations has been carried out towards the other two sources, IRAS 06382+0939 and 19118+0945. M96 found a lower incidence of ammonia emission towards their Low sample than the High one and suggested that the Low sample contains sources in two different evolutionary stages. Some sources are in a stage before the existence of an UCHII region and others are in a stage after the HII region has evolved. 
The sources with evolved HII regions were suggested to be a very late HMPO stage and are expected to be associated with relatively small amounts of neutral gas. The absence of detectable ammonia towards IRAS 18408-0348, 19118+0945 (M 96), 18454-0158 and 18540+0220 (S02) but the presence of only $\mathrm{OH}$ masers suggest that these objects could be relatively evolved.

\section{4. $1720 \mathrm{MHz}$ only sources}

There are five sources, IRAS $04579+4703^{4}, 18264-1152$, $18553+0414,19220+1432$ and $20188+3928$, which have $\mathrm{OH}$ maser emission, but only in the $1720 \mathrm{MHz}$ transition. These sources, apart from IRAS 19220+1432, are associated with other maser types. They are, apart from IRAS $04579+4703$, also associated with, at most, weak radio continuum emission $(<1$ to $11 \mathrm{mJy}$ ). Isolated $1720-\mathrm{MHz}$ masers are sometimes observed in star-forming regions in association with $6668-\mathrm{MHz}$ methanol masers (e.g. Etoka et al. 2005), an association which can be understood in terms of common pumping conditions (Cragg et al. 2002). Other objects associated with only 1720-MHz OH maser emission are believed to be supernova remnants. Elitzur (1976) suggests that the level inversion of these $1720 \mathrm{MHz}$ masers is due to collisional excitation. Models suggest that the masers originate in the post-shock molecular gas behind C-type shocks (e.g. Lockett et al. 1999). However, these 4 sources appear to be star-forming regions. If the Elitzur (1976) model is correct, the masers may be tracing post-shock gas towards these sources. Confirmation of this interpretation will require detailed followup of these objects.

\section{Conclusions}

The results of this survey confirm that $\mathrm{OH}$ masers are common, not only towards HII and UCHII regions as previously known, but also towards less evolved high mass young stars which, although luminous, have not yet significantly ionised their surroundings. Indeed towards these young sources $\mathrm{OH}$ masers are as common as the $\mathrm{H}_{2} \mathrm{O}$ and Class II $\mathrm{CH}_{3} \mathrm{OH}$ masers which have often been been cited as evidence of the youth of these sources. Compared to the $\mathrm{OH}$ masers associated with HII/UCHII regions, the $\mathrm{OH}$ masers towards these younger sources are weaker, suggesting that the $\mathrm{OH}$ maser flux density could be a crude evolutionary indicator.

There is no evidence within the observed sample for a luminosity difference between those sources with $\mathrm{OH}$ masers and those without masers. However, on average the sources with $\mathrm{OH}$ masers have significantly redder [100-12] and [60-12] IRAS colours than those which do not have $\mathrm{OH}$ masers, suggesting that the $\mathrm{OH}$ maser sources are more deeply embedded and hence younger. Similar colour differences are also seen for sources with $\mathrm{H}_{2} \mathrm{O}$ and $\mathrm{CH}_{3} \mathrm{OH}$ masers. However intercomparing the distribution of these colours for sources with each type maser shows them to be indistinguishable. For the sources in this study at least, there is no evidence that one or other of the types of maser in particular traces younger (or older) sources.

The velocity range of the maser emission suggests that the water maser sources may be divided into two groups, one of which shows narrow velocity spread of less than $4-5 \mathrm{~km} \mathrm{~s}^{-1}$, while the other shows a much wider one, with sources which have $\mathrm{H}_{2} \mathrm{O}$ maser velocities spread over up to $\sim 65 \mathrm{~km} \mathrm{~s}^{-1}$. This

${ }^{4}$ Note that the masers towards IRAS $04579+4703$ are offset from the IRAS source. division may well reflect the suggestion by Torrelles et al. (1997, 1998) that $\mathrm{H}_{2} \mathrm{O}$ masers can trace both molecular outflows and accretion disks perpendicular to the molecular outflow.

Comparing the $\mathrm{OH}$ and methanol maser peak fluxes, the majority of the sources are either methanol-favoured or $\mathrm{OH}$-favoured with over $40 \%$ of the sources in each of these categories. Both the detection rate and velocity coverage of the maser emission suggests a close association between $\mathrm{OH}$ and $\mathrm{CH}_{3} \mathrm{OH}$ masers, which has also been reported by other authors (e.g. Caswell 1998, and references therein; Szymczak \& Kus 2000; Szymczak \& Gérard 2004). However understanding the exact origin of the $\mathrm{OH}$ masers and the detailed connection between the $\mathrm{OH}$ and $\mathrm{CH}_{3} \mathrm{OH}$ masers requires higher spatial resolution follow-up studies such as that of IRAS $20126+4104$ by Edris et al. (2005). High resolution observations of some molecular outflow sources have shown that towards HMPOs the $\mathrm{OH}$ masers often arise from a circumstellar disc around the central source (Hutawarakorn \& Cohen 1999; Hutawarakorn et al. 2002; Hutawarakorn et al. 2003; Edris et al. 2005). Such high resolution follow-up of the sources in this survey may therefore identify additional HMPOs with circumstellar disks and making use of the Zeeman sensitivity of the $\mathrm{OH}$ emission, measure the magnetic field within them.

Acknowledgements. We would like to thank the staff at both Nançay and Green Bank for their invaluable help in obtaining the data presented here. We would also like to thank Malcolm Gray for helpful discussions. K.A.E and G.A.F would like to dedicate this paper to the memory of R.J. Cohen who passed away on 1 November 2006.

\section{References}

Argon, A. L., Reid, M. J., \& Menten, K. M. 2000, ApJS, 129, 159 Benson, J. M., \& Johnston, K. J. 1984, ApJ, 277, 181

Beuther, H., Walsh, A., Schilke, P., et al. 2002a, A\&A, 390, 289 Beuther, H., Sridharan, T. K., Schilke, P., et al. 2002b, A\&A, 383, 892 Beuther, H., Schilke, P., Menten, K. M., et al. 2002c, ApJ, 566, 945 Beuther, H., Schilke, P., Gueth, F., et al. 2002d, A\&A, 387, 931 Beuther, H., Schilke, P., \& Gueth, F. 2004, ApJ, 608, 330

Beuther, H., Zhang, Q., Sridharan, T. K., \& Chen, J. 2005, ApJ, 628, 800 Bourke, T. L., Hyland, A. R., \& Robinson, G. 2005, ApJ, 625, 883 Braz, M. A., Lepine, L. R. D., Sivagnanam, P., \& Le Squeren, A. M. 1990, A\&A, 236, 479

Brebner, G. C. 1988, Ph.D. Thesis University of Manchester

Bronfman, L., Nyman, L. A., \& May, J. 1996, A\&AS, 115, 81

Caswell, J. L. 1996, MNRAS, 279, 79

Caswell, J. L. 1997, MNRAS, 289, 203

Caswell, J. L. 1998, MNRAS, 297, 215

Caswell, J. L., \& Haynes, R. F. 1983, Aust. J. Phys., 36, 361

Caswell, J. L., Vaile, R. A., \& Forster, J. R. 1995, MNRAS, 277, 210

Cesaroni, R., Felli, M., Testi, L., Walmsley, C. M., \& Olmi, L. 1997, A\&A, 325, 725 (C97)

Churchwell, E. 2002, ARA\&A, 40, 27

Cohen, R. J. 1989, Rep. Prog. Phys., 52, 881

Cohen, R. J., Rowland, P. R., \& Blair, M. M. 1984, MNRAS, 210, 425

Cohen, R. J., Baart, E. E., \& Jonas, J. L. 1988, MNRAS, 231, 205

Cohen, R. J., Brebner, G. C., Hutawarakorn, B., \& Gasiprong, N. 2003, IAU General Assembly, 2003 IAUS, 221P, 168C

Cragg, D. M., Sobolev, A. M., \& Godfrey, P. D. 2002, MNRAS, 331, 521

Cragg, D. M., Sobolev, A. M., \& Godfrey, P. D. 2005, MNRAS, 360, 533

Edris, K. A., Fuller, G. A., Cohen, R. J., \& Sandra, E. 2005, A\&A, 343, 213

Elitzur, M. 1976, ApJ, 203, 124

Elitzur, M., \& de Jong, T. 1978, A\&A, 67, 323

Etoka, S., Cohen, R. J., \& Gray, M. D. 2005, MNRAS, 360, 1162

Foster, J. R., \& Caswell, J. L. 2000, ApJ, 530, 371

Galt, J. 2004, AJ, 127, 3479

Garay, G., \& Lizano, S. 1999, PASP, 111, 1049

Genzel, R., \& Downes, D. 1977, A\&AS, 30, 145

Gray, M. D., Field, D., \& Doel, R. C. 1992, A\&A, 262, 555

Haynes, R. F., Caswell, J. L., \& Simons, L. W. J. 1979, Aust. J. Phys. Astrophys. Suppl., 48, 1 
Hofner, P., Cesaroni, R., Rodríguez, L. F., \& Marti, J. 1999, A\&A, 345, 43 Hughes, V. A., \& Macleod, G. C. 1993, AJ, 105, 1495

Hutawarakorn, B., \& Cohen, R. J. 1999, MNRAS, 303, 845

Hutawarakorn, B., \& Cohen, R. J. 2003, MNRAS, 345, 175

Hutawarakorn, B., Cohen, R. J., \& Brebner, G. C. 2002, MNRAS, 330, 349

Jenness, T., Scott, P. F., \& Padman, R. 1995, MNRAS, 276, 1024

Lada, C. J. 1976, ApJS, 32, 603

Lockett, P., Gauthier, E., \& Elitzur, M. 1999, ApJ, 447, 211

Minier, V., Conway, J. E., \& Booth, R. S. 2001, A\&A, 369, 278

Minier, V., Burton, M. G., Hill, T., et al. 2005, A\&A, 429, 945

Miralles, M. P., Rodríguez, L. F., \& Scalise, E. 1994, ApJS, 92, 173

Molinari, S., Brand, J., Cesaroni, R., \& Palla, F. 1996, A\&A, 308, 573

Molinari, S., Brand, J., Cesaroni, R., Palla, F., \& Palumbo, G. G. C. 1998, A\&A, 336, 339

Molinari, S., Testi, L., Rodríguez, L., \& Zhang, Q. 2002, ApJ, 570, 758

Moore, T. J. T., Mountain, C. M., Yamashita, T., \& Selby, M. J. 1988, MNRAS, 234,95

Moscadelli, L., Cesaroni, R., \& Rioja, M. J. 2000, A\&A, 360, 663 (MCR)

Palla, F., Brand, J., Cesaroni, R., Comoretto, G., \& Felli, M. 1991, A\&A, 246, 249 (P91)

Pestalozzi, M. R., Minier, V., \& Booth, R. S. 2005, A\&A, 432, 737

Schutte, A. J., Van der Walt, D. J., Gaylard, M. J., \& Macleod, G. C. 1993 , MNRAS, 261, 783
Shepherd, D. S., Nürnberger, D. E. A., \& Bronfman, L. 2004, ApJ, 602, 850 Simon, M., Cassar, L., Felli, M., et al. 1984, ApJ, 278, 170

Slysh, V. I., Dzura, A. M., Val'tts, I. E., \& Gérard, E. 1994, A\&AS, 106, 87

Slysh, V. I., Dzura, A. M., Val'tts, I. E., \& Gérard, E. 1997, A\&AS, 124, 85

Slysh, V. I., Val'tts, I. E., Kalenskii, S. V., et al. 1999, A\&AS, 134, 115

Sridharan, T. K., Beuther, H., Schilke, P., Menten, K. M., \& Wyrowski, F. 2002, ApJ, 566, 931

Szymczak, M., \& Gérard, E. 2004, A\&A, 414, 235

Szymczak, M., \& Kus, A. J. 2000, A\&AS, 147, 181

Szymczak, M., Hrynek, G., \& Kus, A. J. 2000a, A\&AS, 143, 269 (SHK2000)

Szymczak, M., Kus, A. J., \& Hrynek, G. 2000b, MNRAS, 312, 211

Tofani, G., Felli, M., Taylor, G. B., \& Hunter, T. R. 1995, A\&AS, 112, 299

Torrelles, J. M., Gómez, J. F., Rodríguez, L. F., et al. 1997, ApJ, 489, 744

Torrelles, J. M., Gómez, J. F., Rodríguez, L. F., et al. 1998, ApJ, 505, 756

Verdes-Montenegro, L., Torelles, J. M., Rodriguez, L. F., et al. 1989, ApJ, 346, 193

Walsh, A. J., Burton, M. G., Hyland, A. R., \& Robinson, G. 1998, MNRAS, 301, 640

Williams, S. J., Fuller, G. A., \& Sridharan, T. K. 2004, A\&A, 417, 115

Wood, D. O. S., \& Churchwell, E. 1989, ApJS, 83, 119 (WC89)

Wu, Y., Wei, Y., Zhao, M., et al. 2004, A\&A, 426, 503

Zhang, Q., Hunter, T. R., \& Sridharan, T. K. 1998, ApJ, 505, 151

Zhang, Q., Hunter, T. R., Brand, J., et al. 2005, ApJ, 625, 864 
K. A. Edris et al.: OH maser survey towards HMPOs, Online Material $p 1$

\section{Online Material}


K. A. Edris et al.: OH maser survey towards HMPOs, Online Material $p 2$
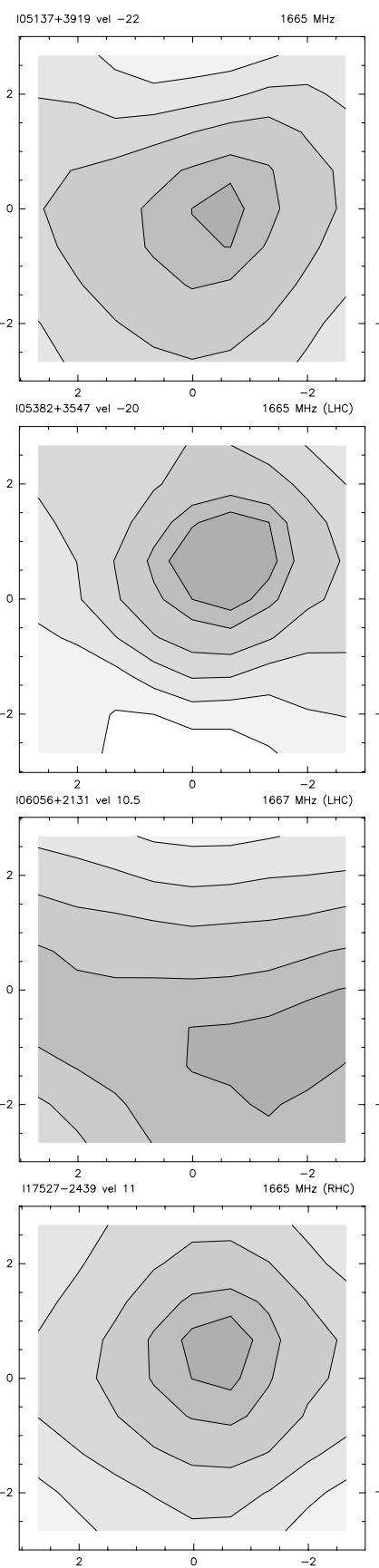
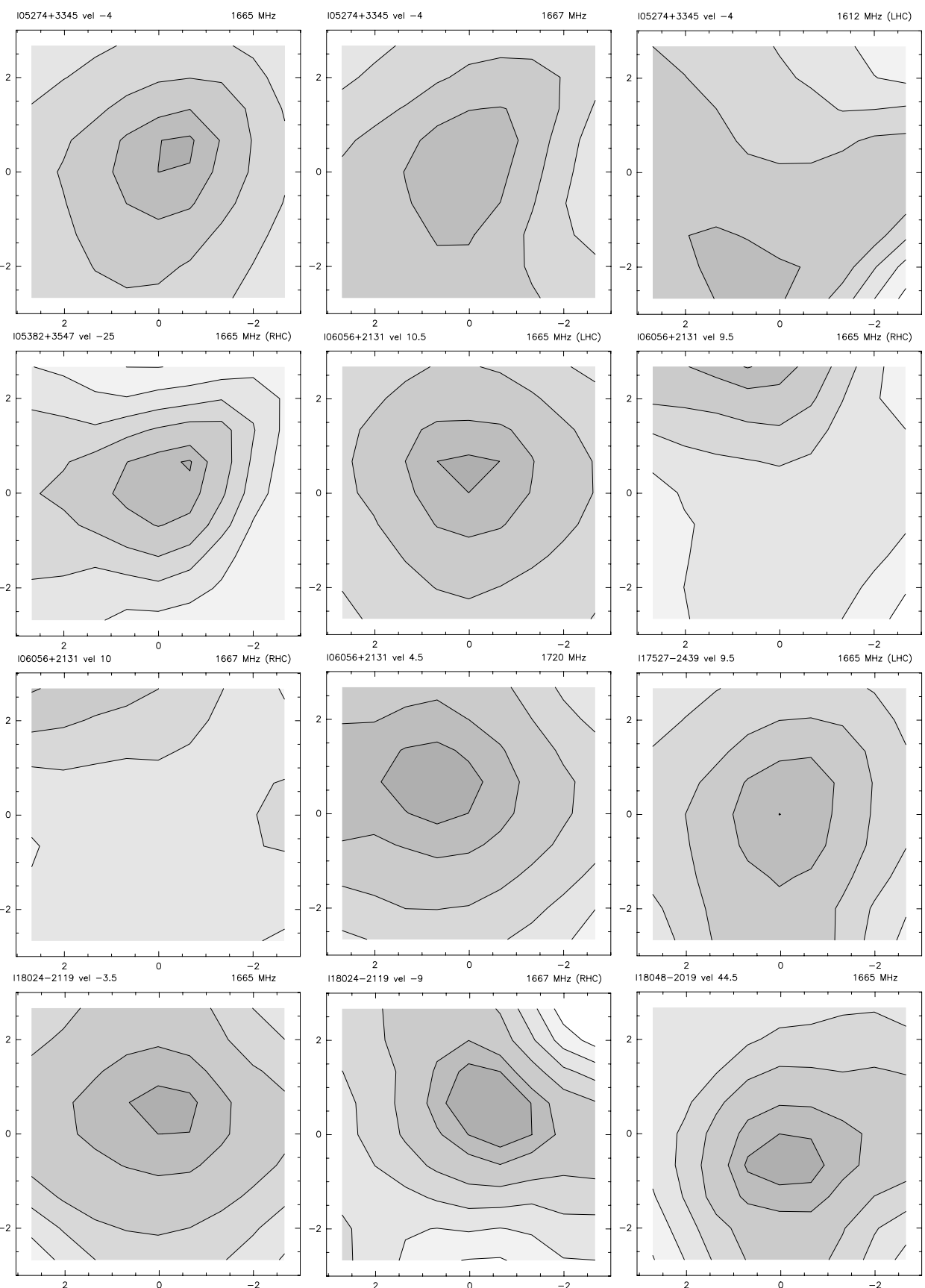

Fig. 17. The 9-point maps of the maser intensity integrated over the velocity range of each maser component detected by the GBT observations. The axes show RA and Dec offset in arcminutes from the IRAS position. The contours levels run from $10 \%$ to $90 \%$ (in $20 \%$ steps) of the peak flux given in Table 2. 
K. A. Edris et al.: OH maser survey towards HMPOs, Online Material p 3
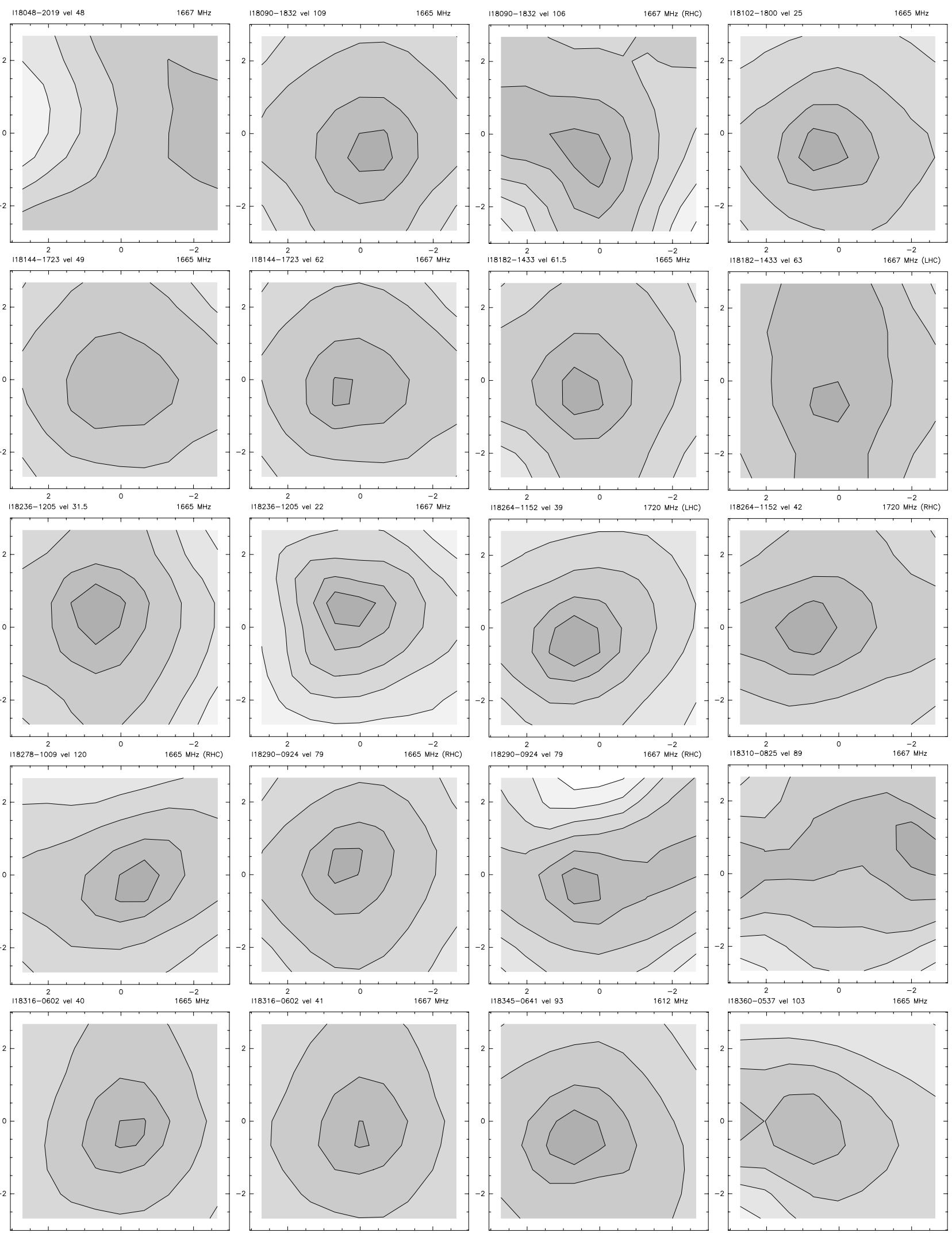

Fig. 17. continued. 
K. A. Edris et al.: OH maser survey towards HMPOs, Online Material p 4
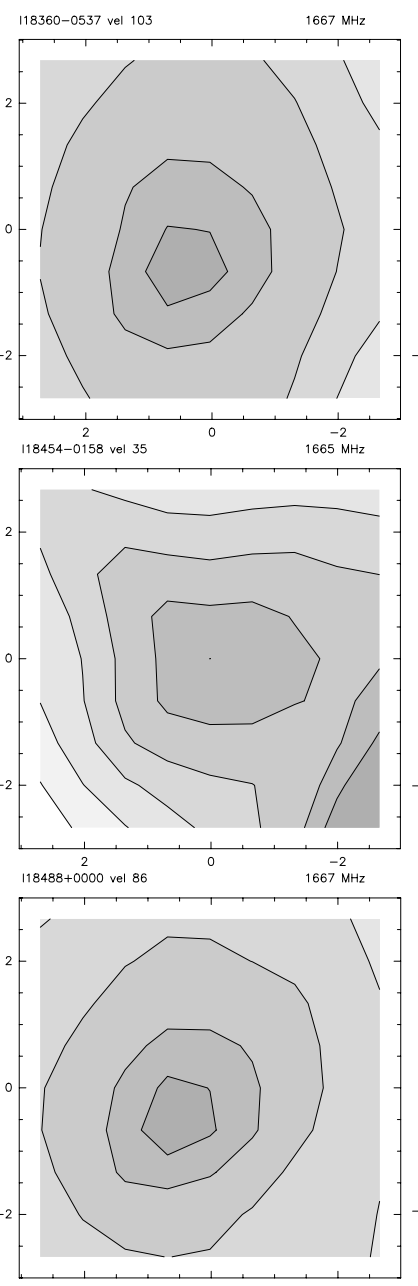

$18527+0301$ vel $74 \quad 1667 \mathrm{MHZ}$ ( LHC
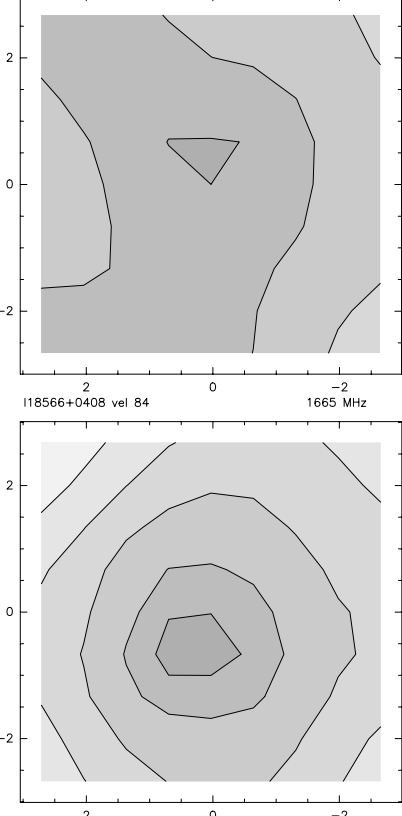

Fig. 17. continued.
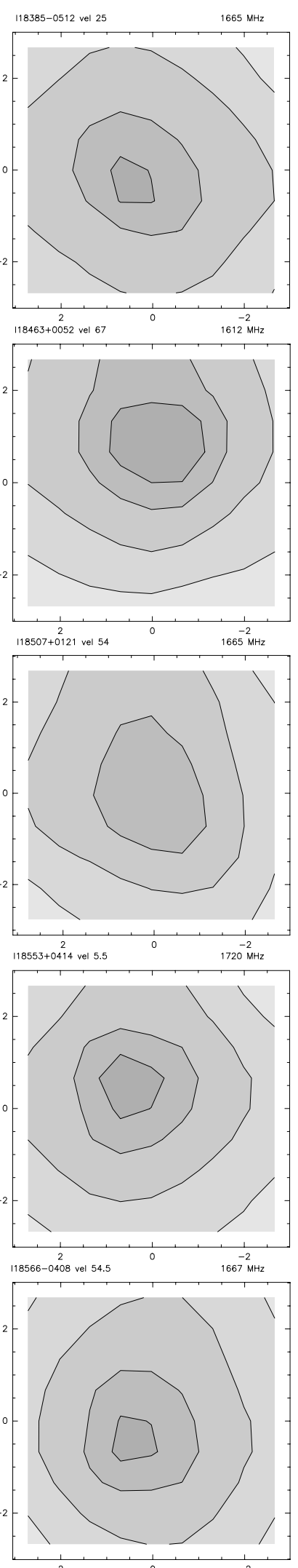
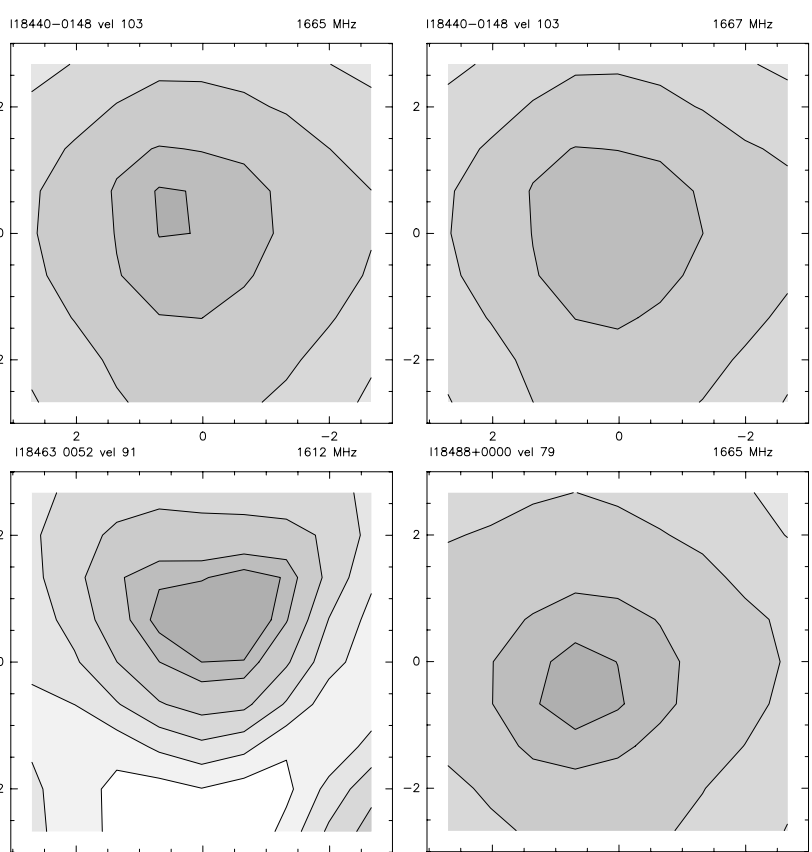

$667^{-2} \mathrm{MHz}$
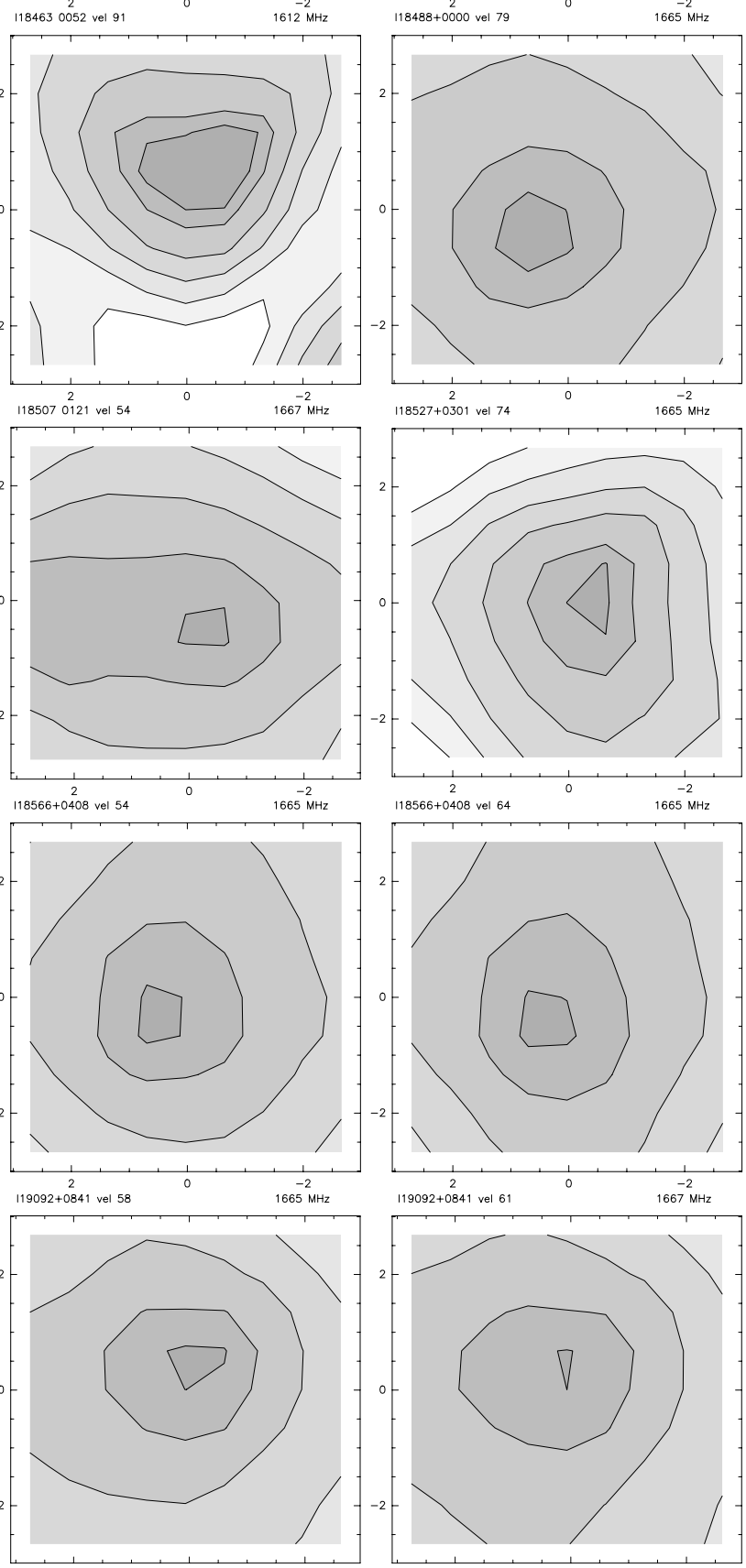

\section{Fig. 17. continued.}


K. A. Edris et al.: OH maser survey towards HMPOs, Online Material p 5
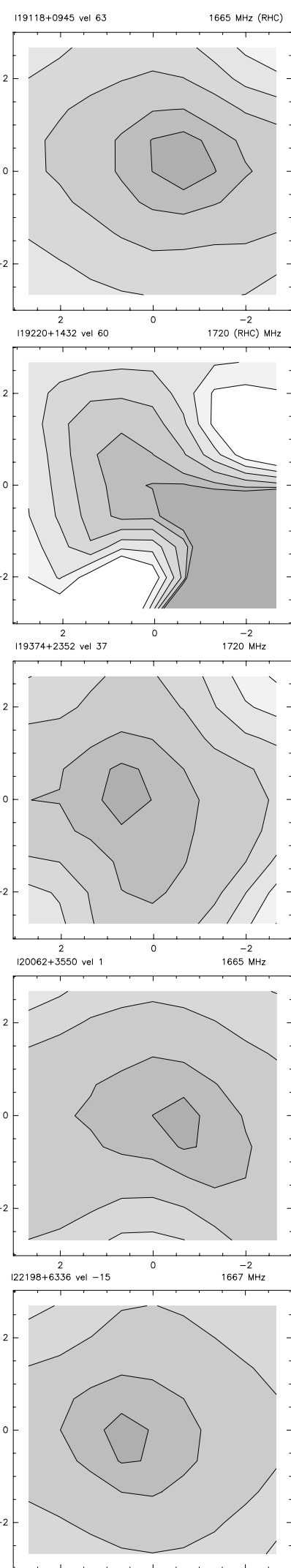

Fig. 17. continued.
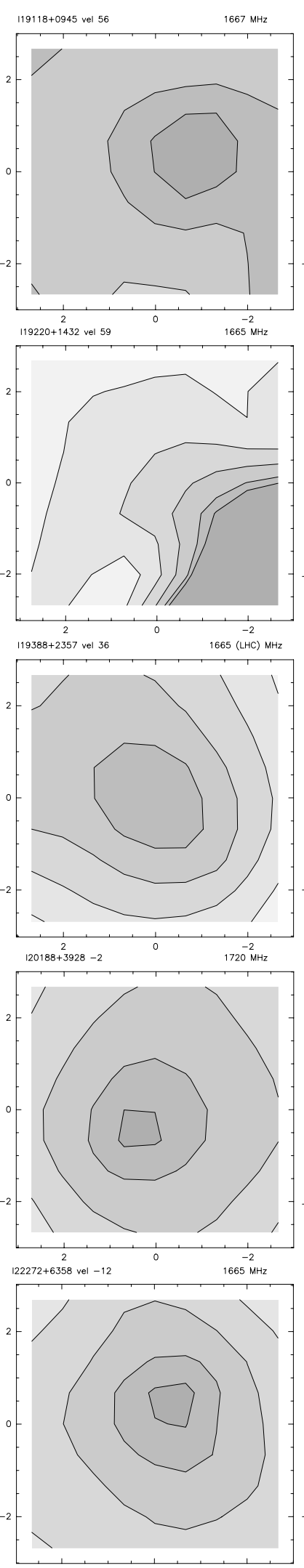
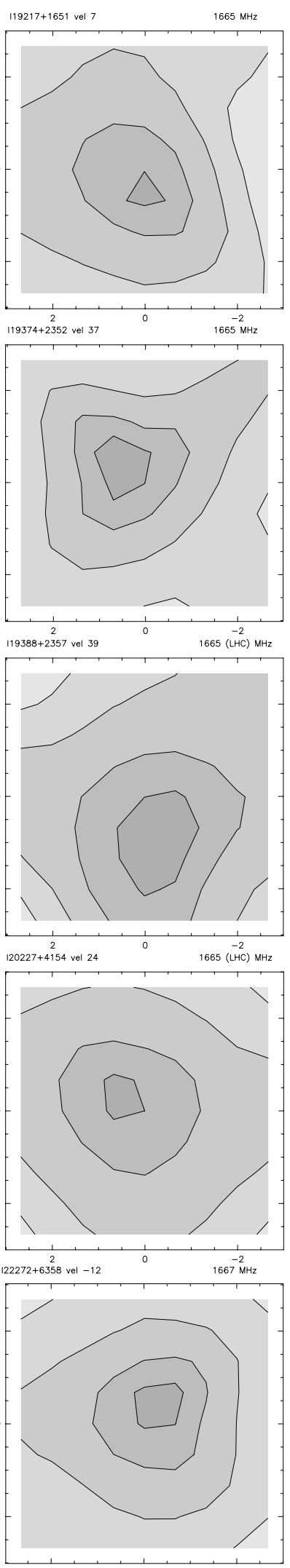
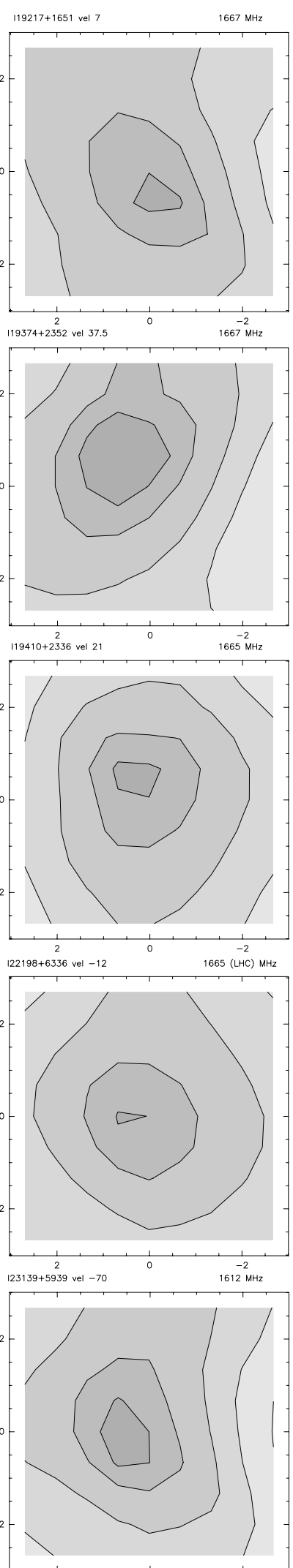
K. A. Edris et al.: OH maser survey towards HMPOs, Online Material p 6
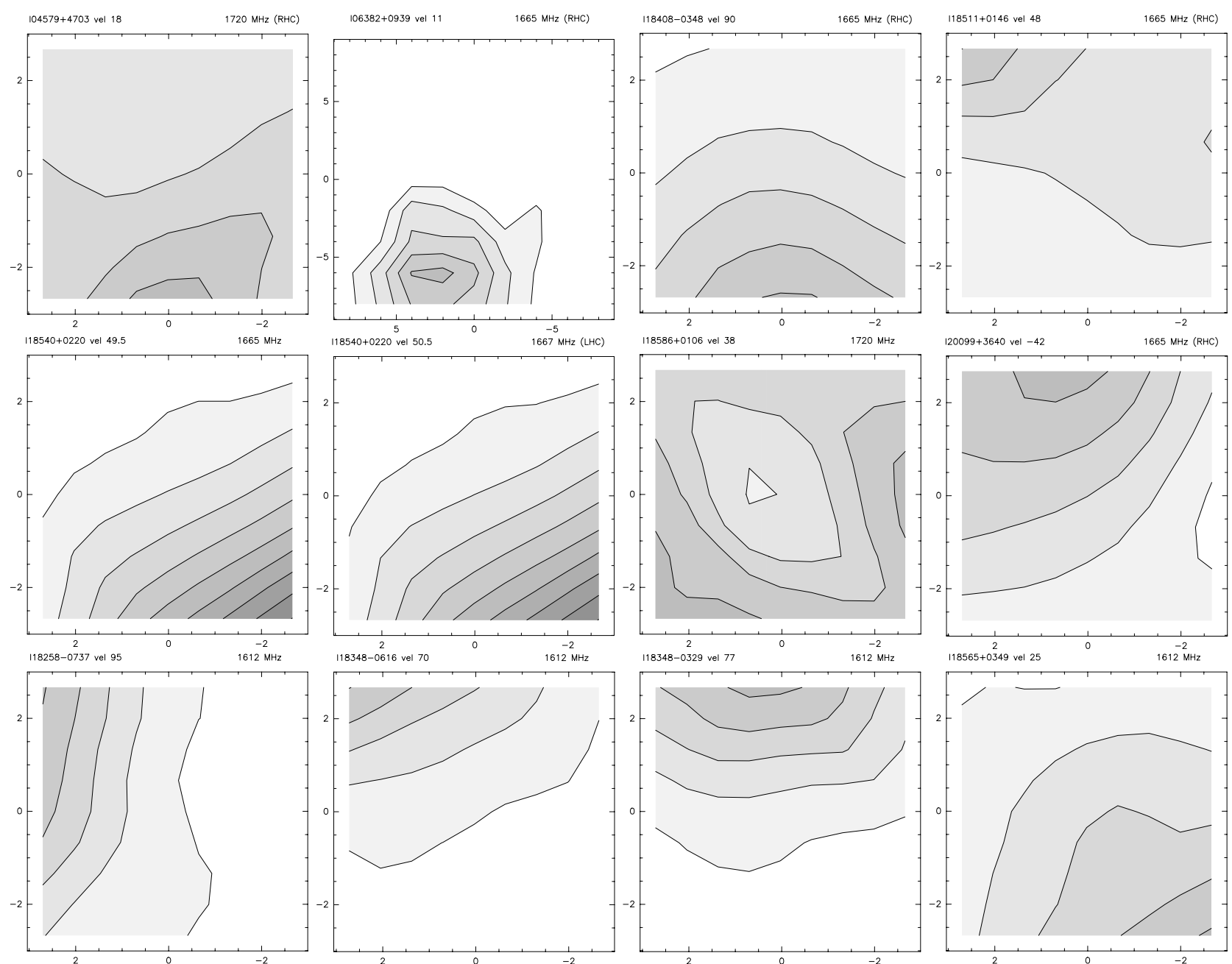

Fig. 18. Same as Fig. 17 but for $\mathrm{OH}$ maser sources offset from the IRAS position by $>2^{\prime}$. 
K. A. Edris et al.: OH maser survey towards HMPOs, Online Material $p 7$

IRAS05137+3919
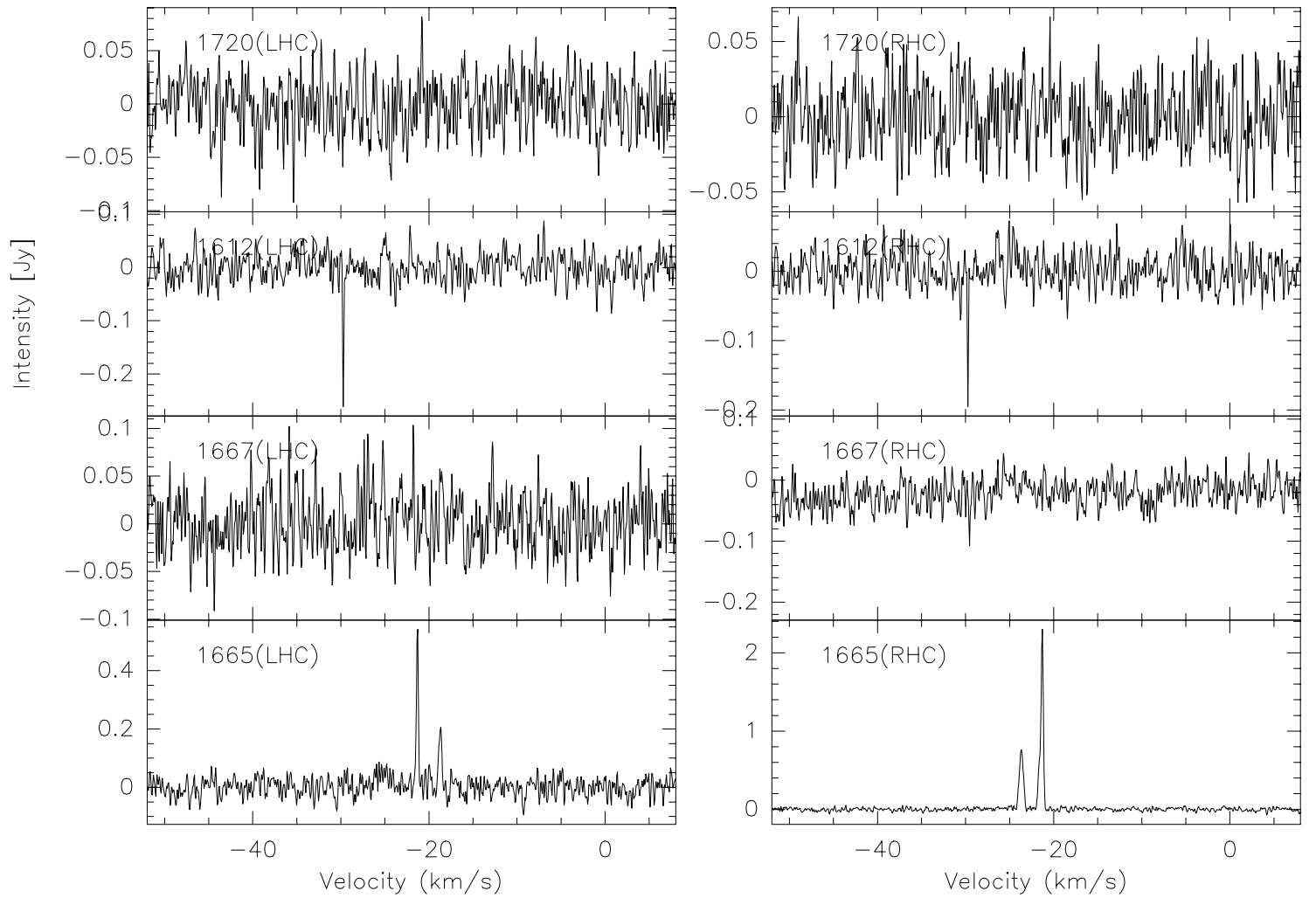

IRAS05274+3345
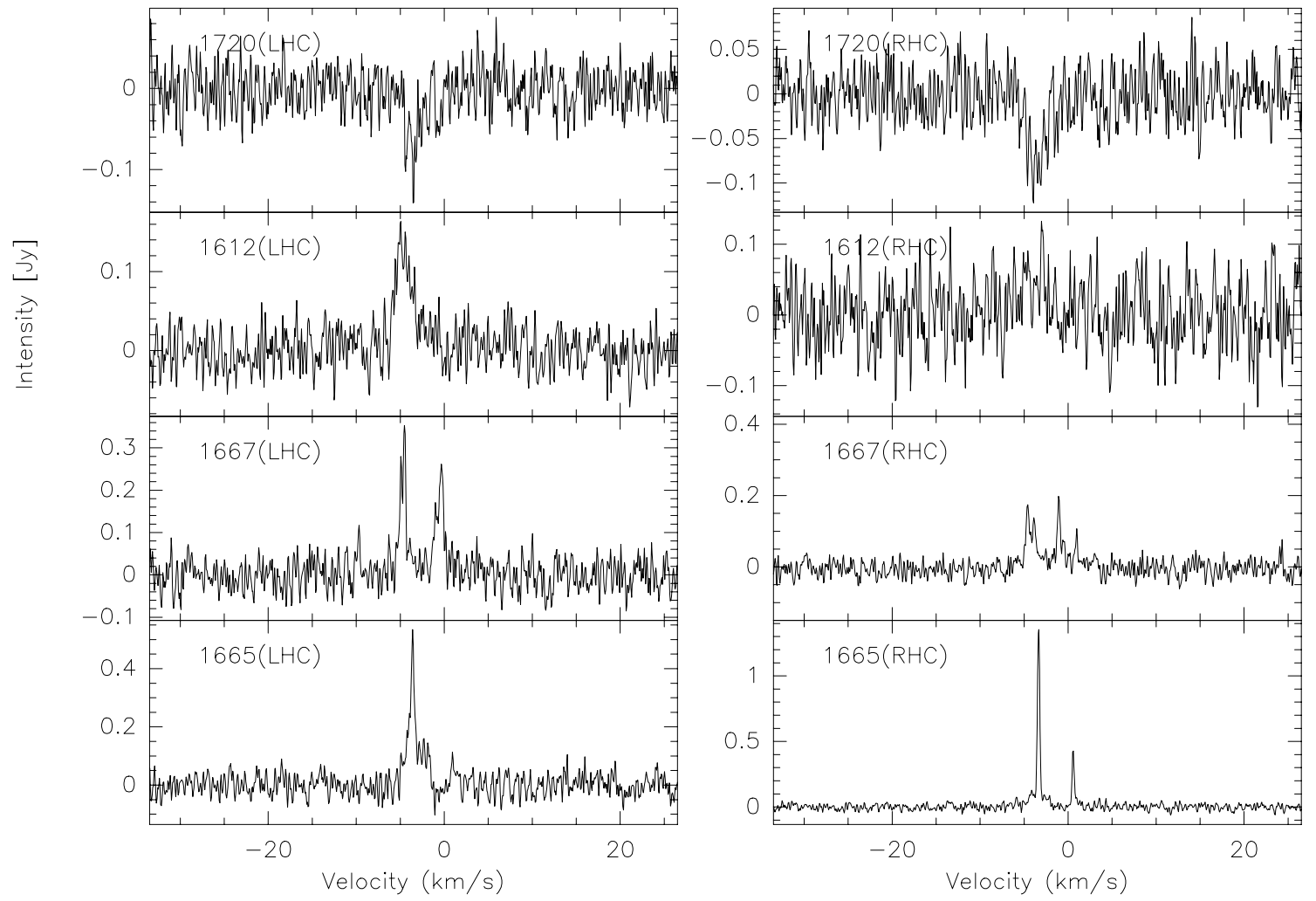

Fig. 19. The spectra of each $\mathrm{OH}$ maser line detected by GBT and Nançay observations. The sources names are given at the top of each group of spectra. The intensities are in Jy. 
K. A. Edris et al.: OH maser survey towards HMPOs, Online Material p 8

IRAS05358+3543
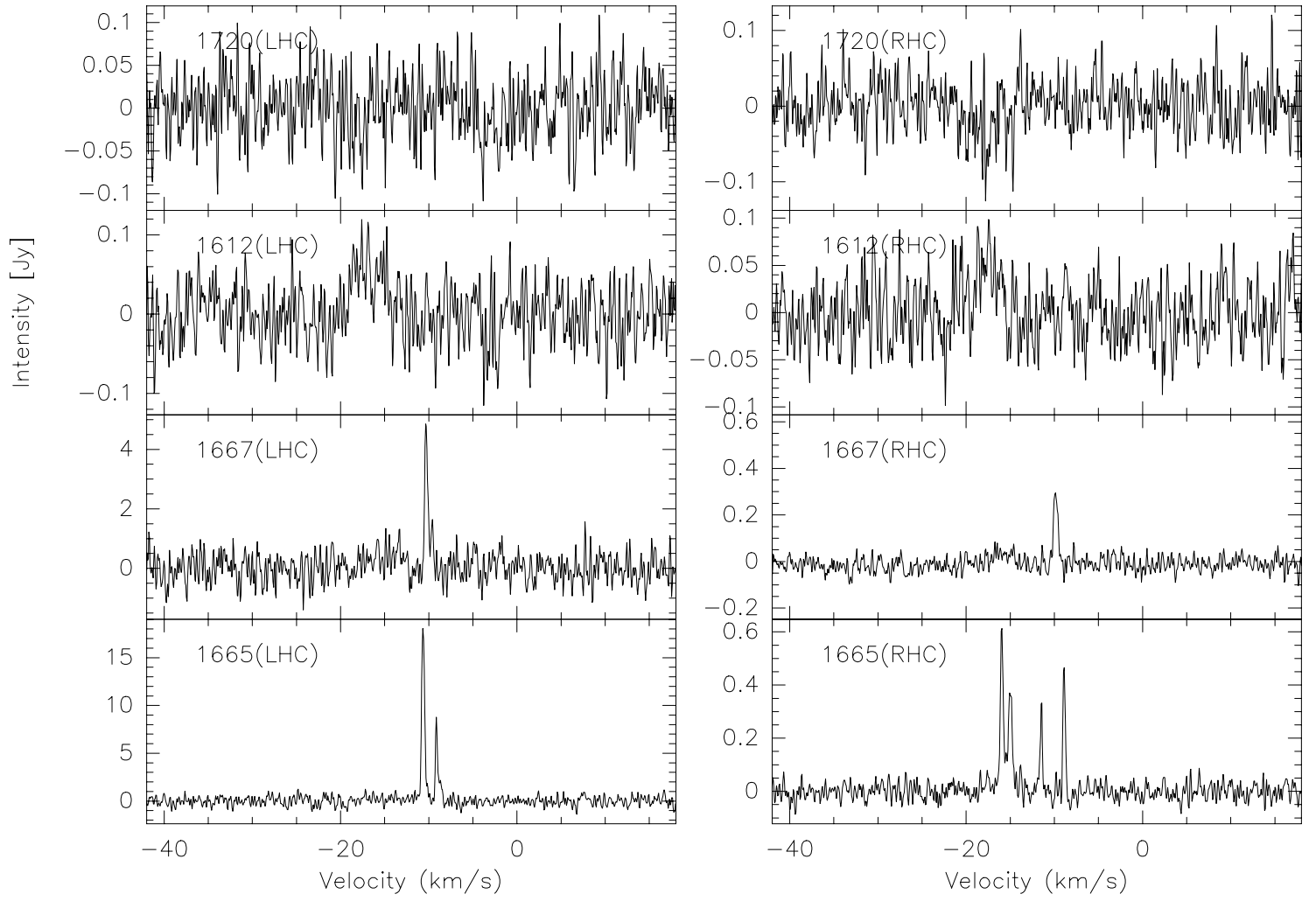

IRAS05382+3547
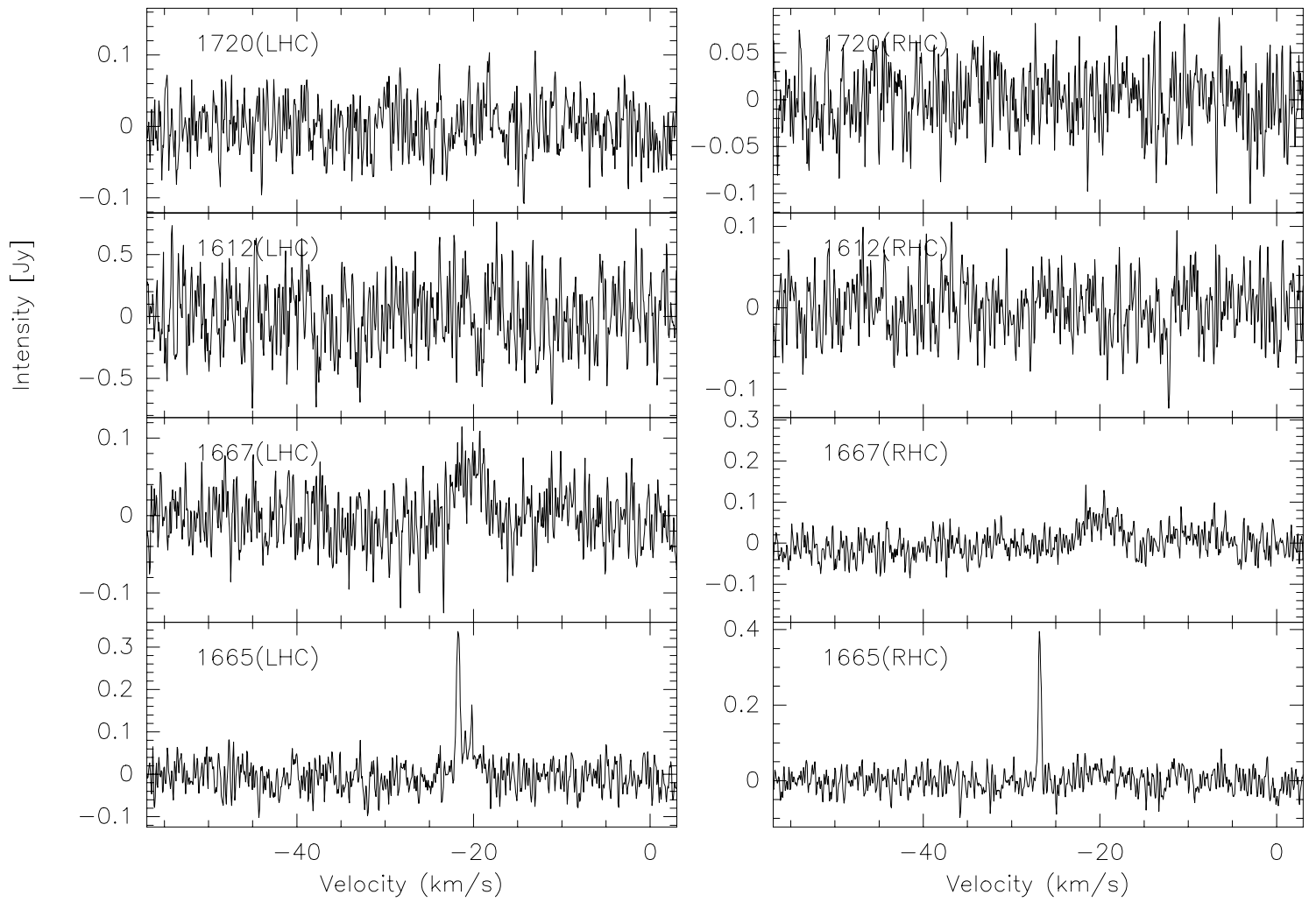

Fig. 19. continued. 
K. A. Edris et al.: OH maser survey towards HMPOs, Online Material $p 9$

IRAS06056+2131
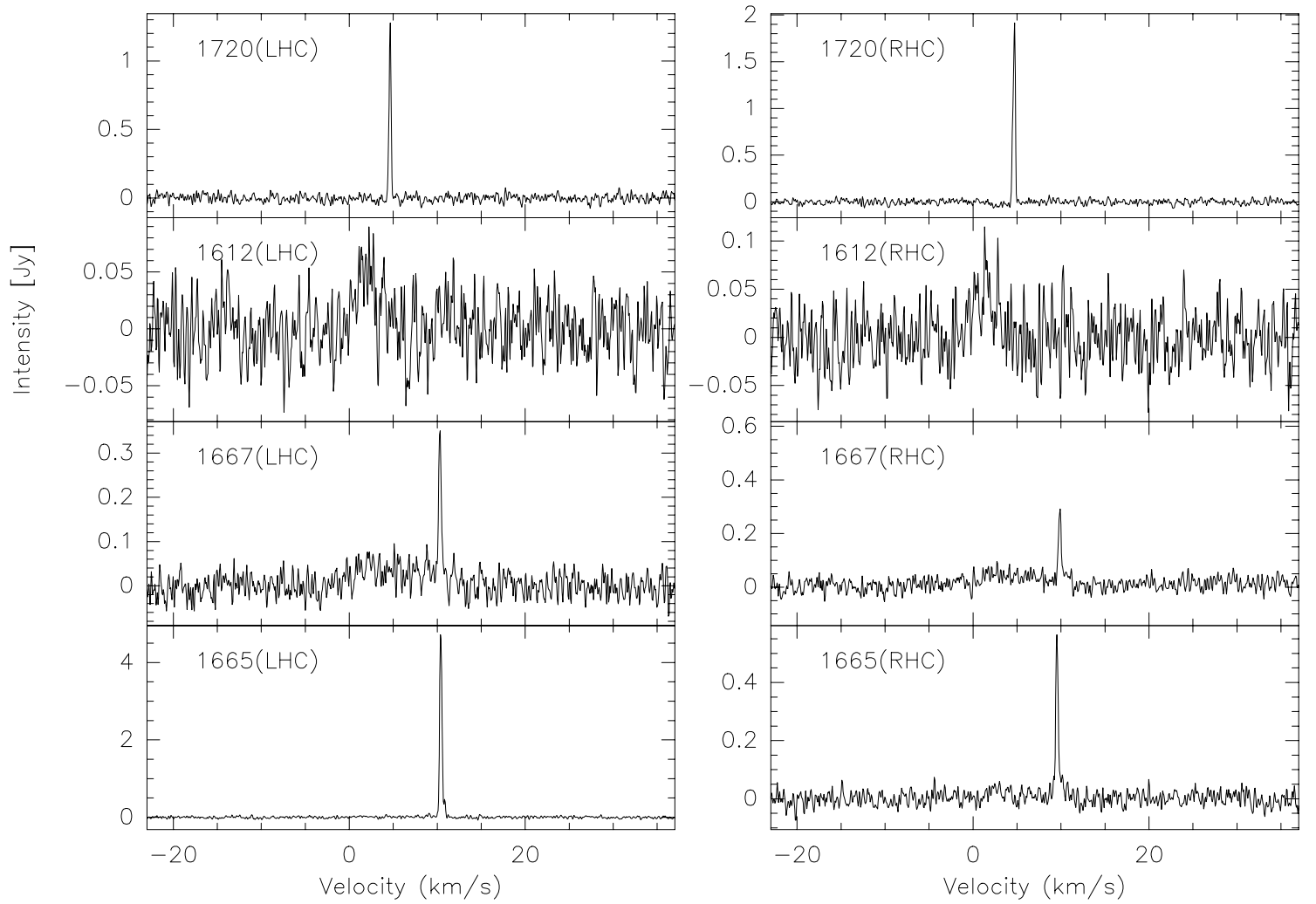

IRAS17527-2439
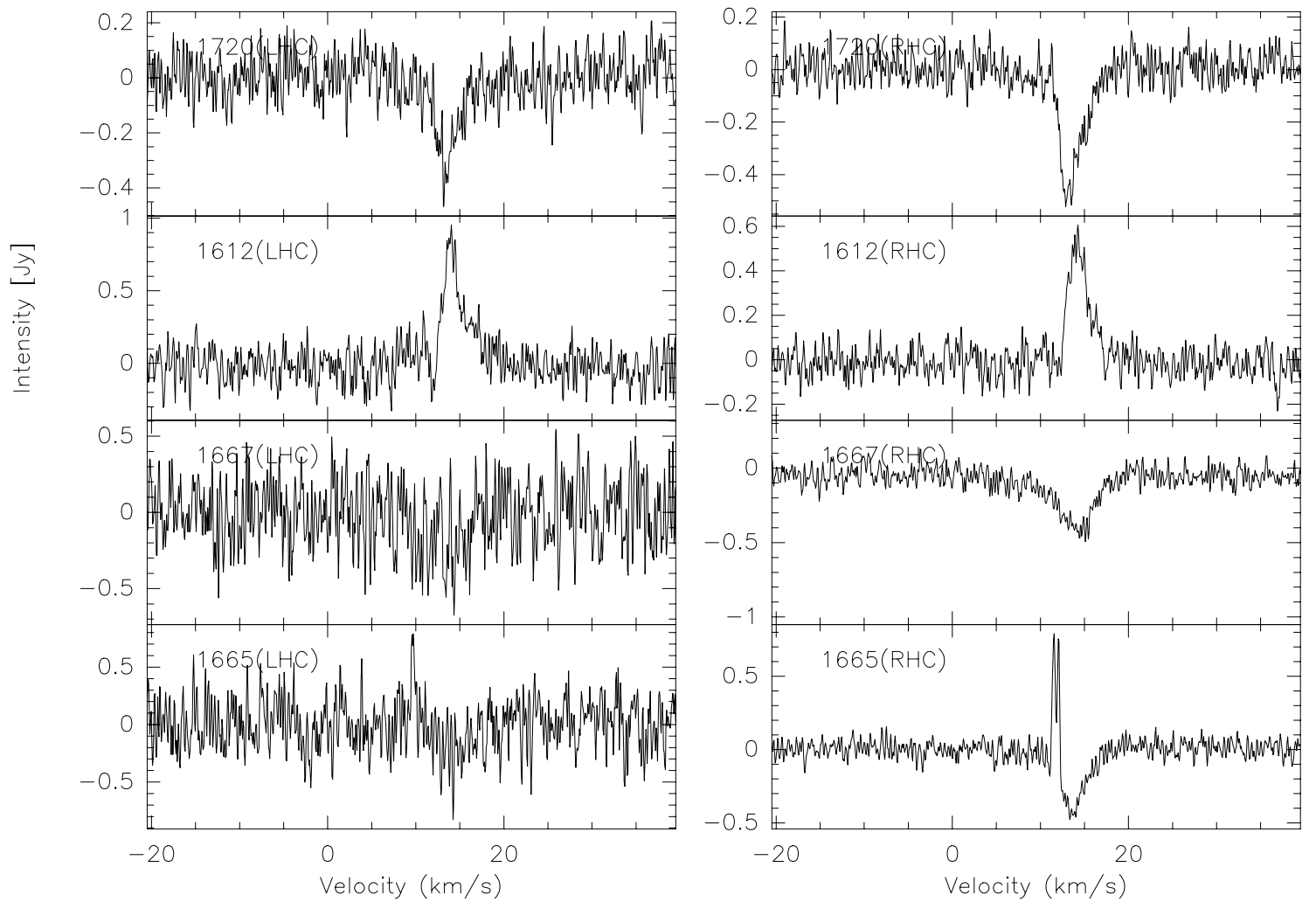

Fig. 19. continued. 
K. A. Edris et al.: OH maser survey towards HMPOs, Online Material p 10 IRAS18018-2426
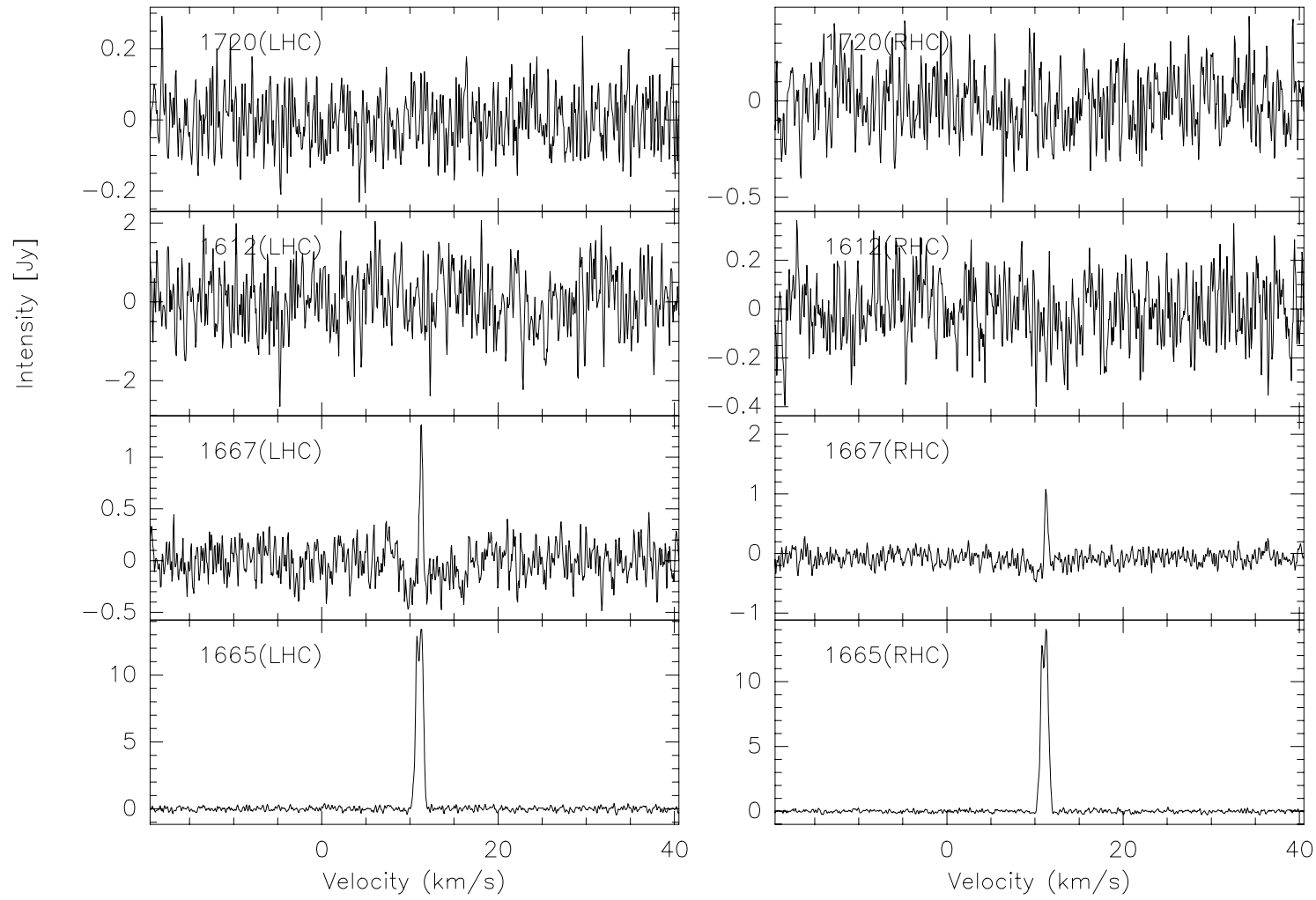

IRAS18024-2119
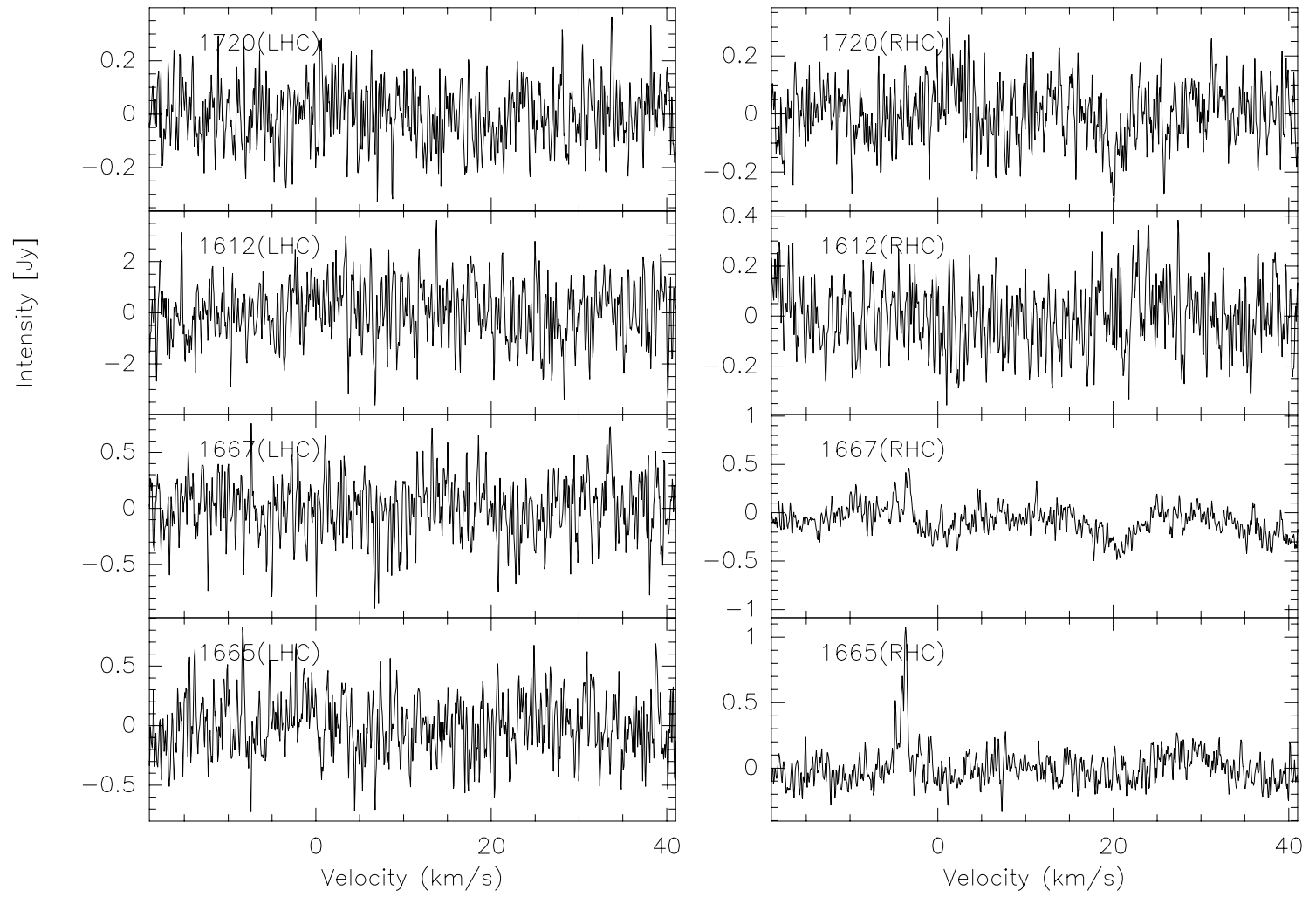

Fig. 19. continued. 
K. A. Edris et al.: OH maser survey towards HMPOs, Online Material p 11

IRAS18048-2019
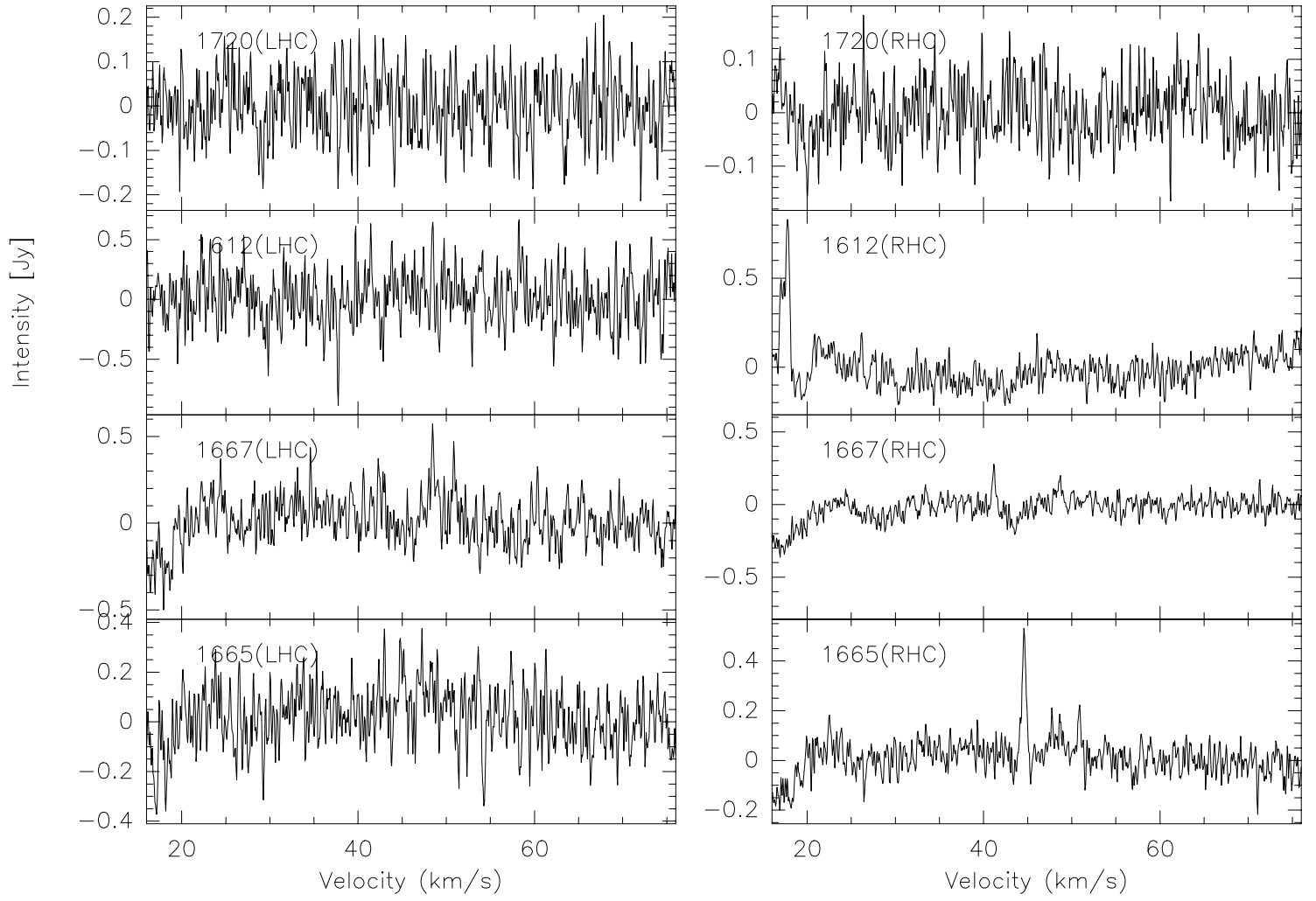

IRAS18089-1732
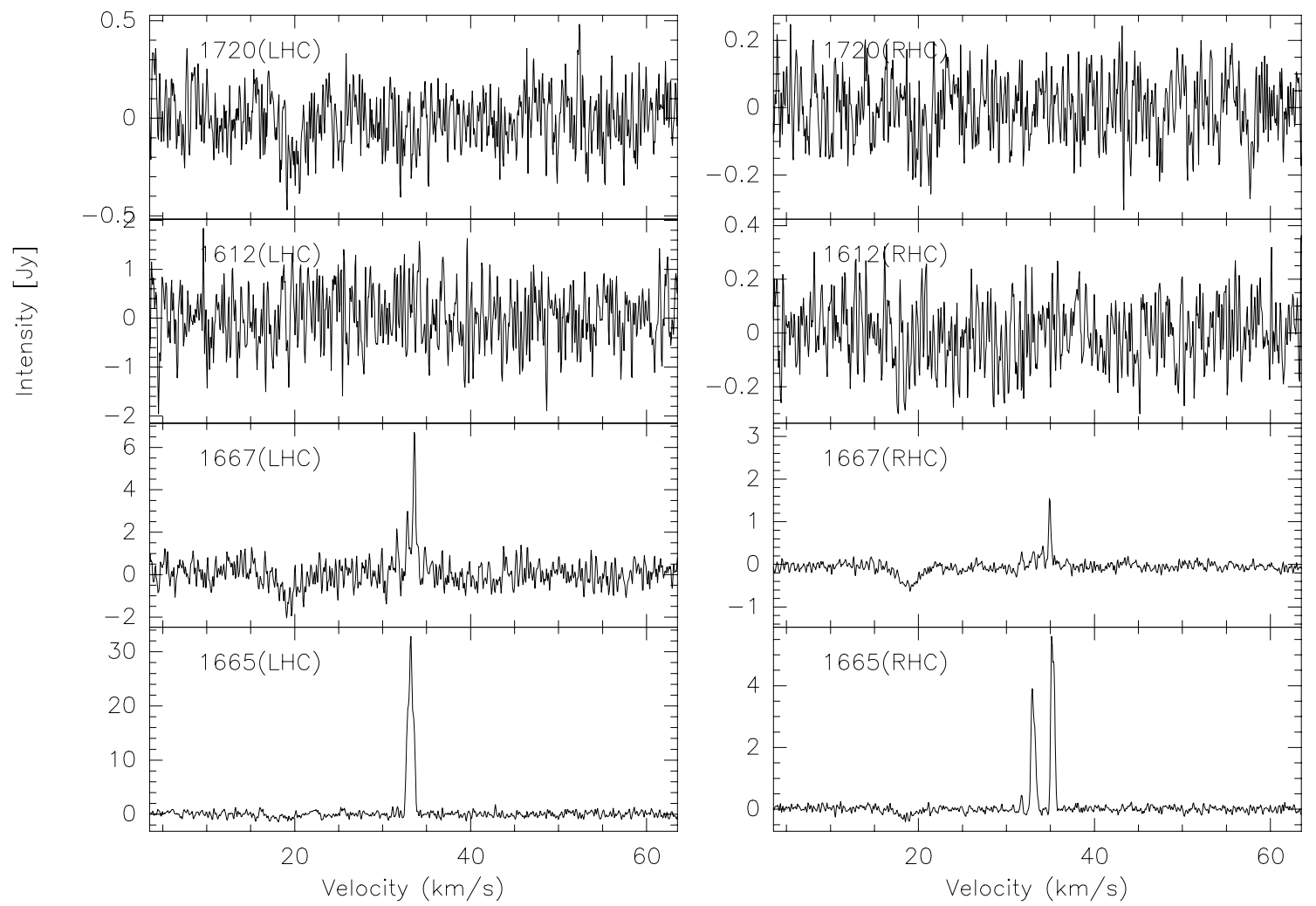

Fig. 19. continued. 
K. A. Edris et al.: OH maser survey towards HMPOs, Online Material p 12

IRAS18090-1832
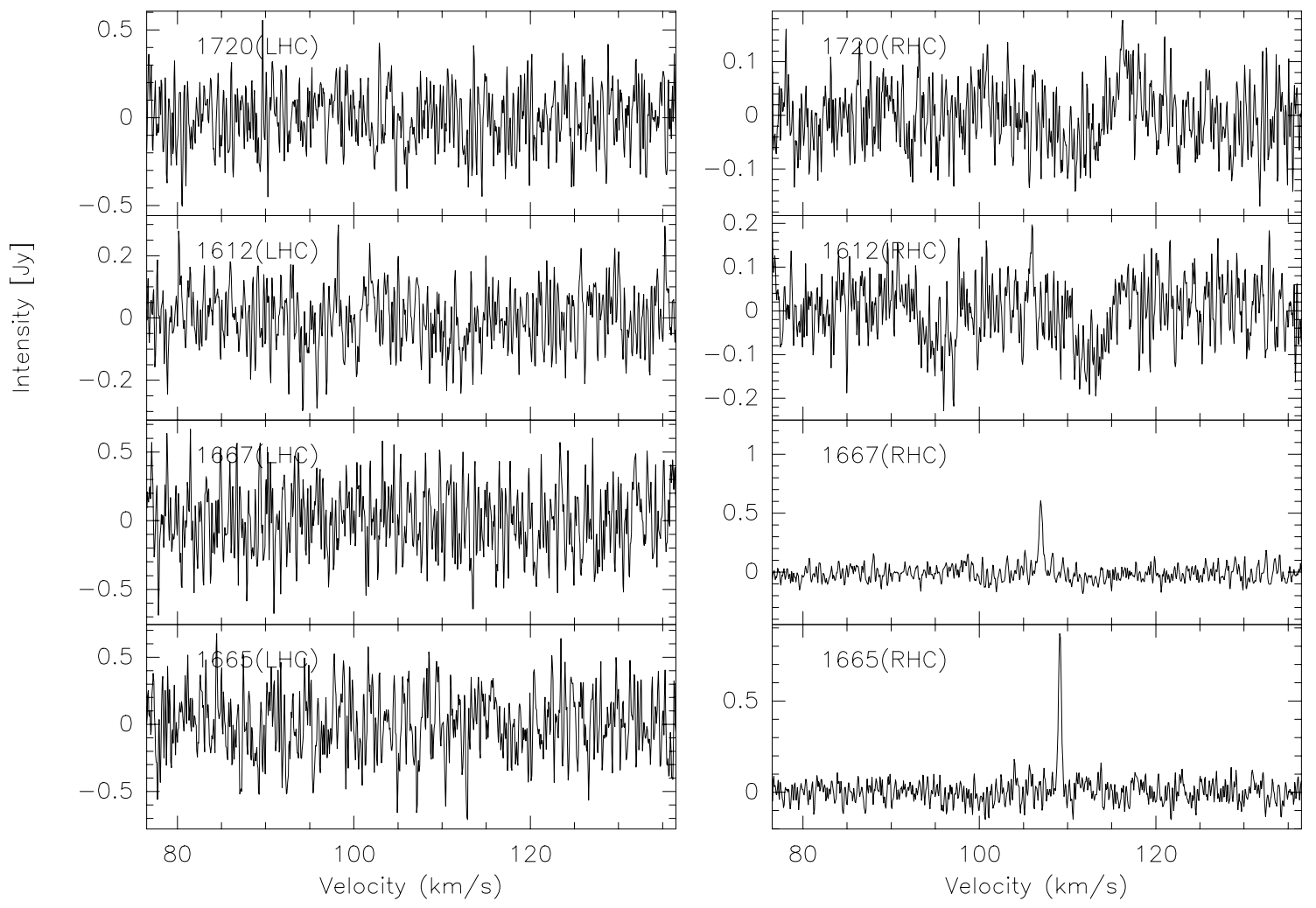

IRAS18102-1800
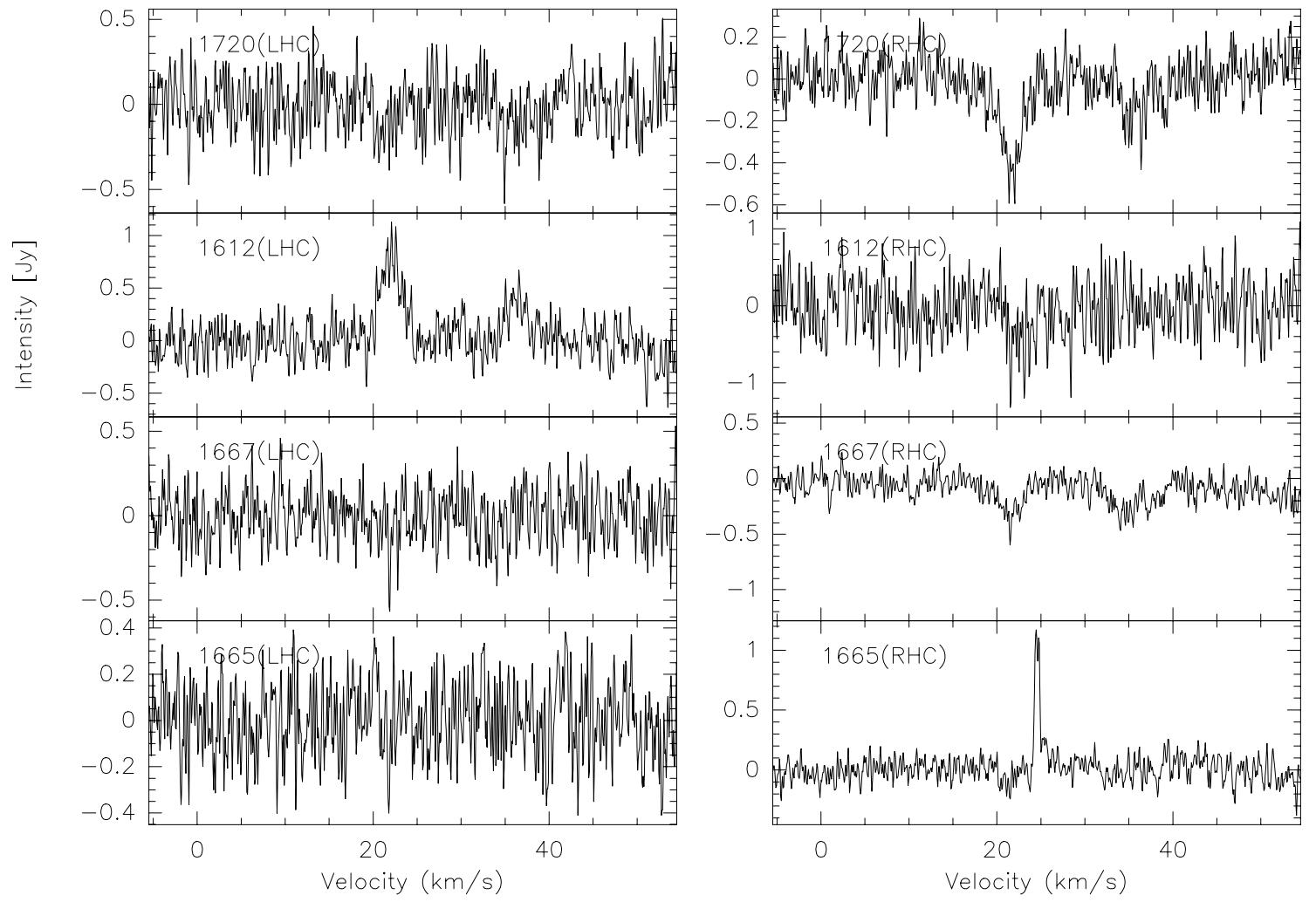

Fig. 19. continued. 
K. A. Edris et al.: OH maser survey towards HMPOs, Online Material p 13

IRAS18144-1723
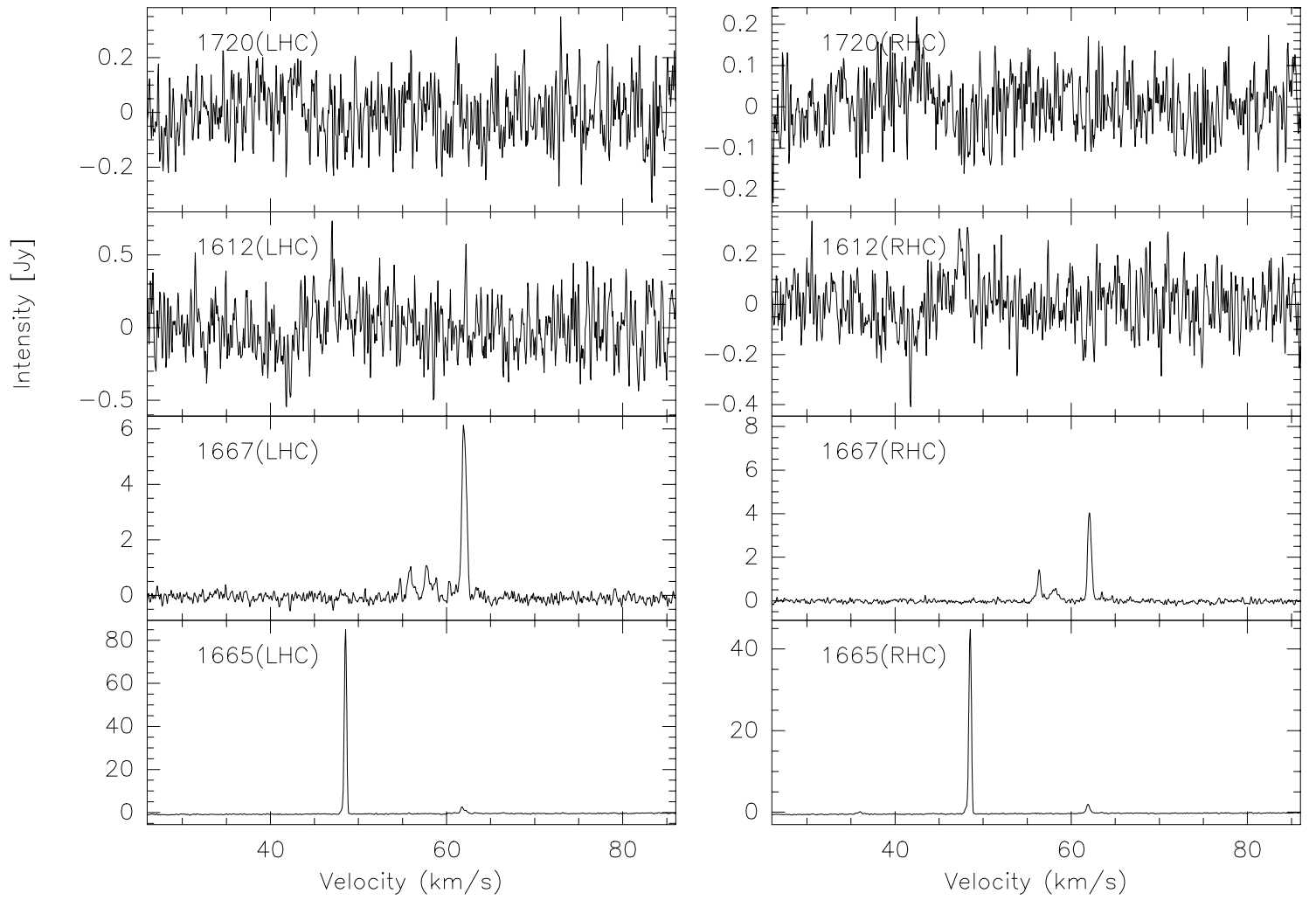

IRAS18182-1433
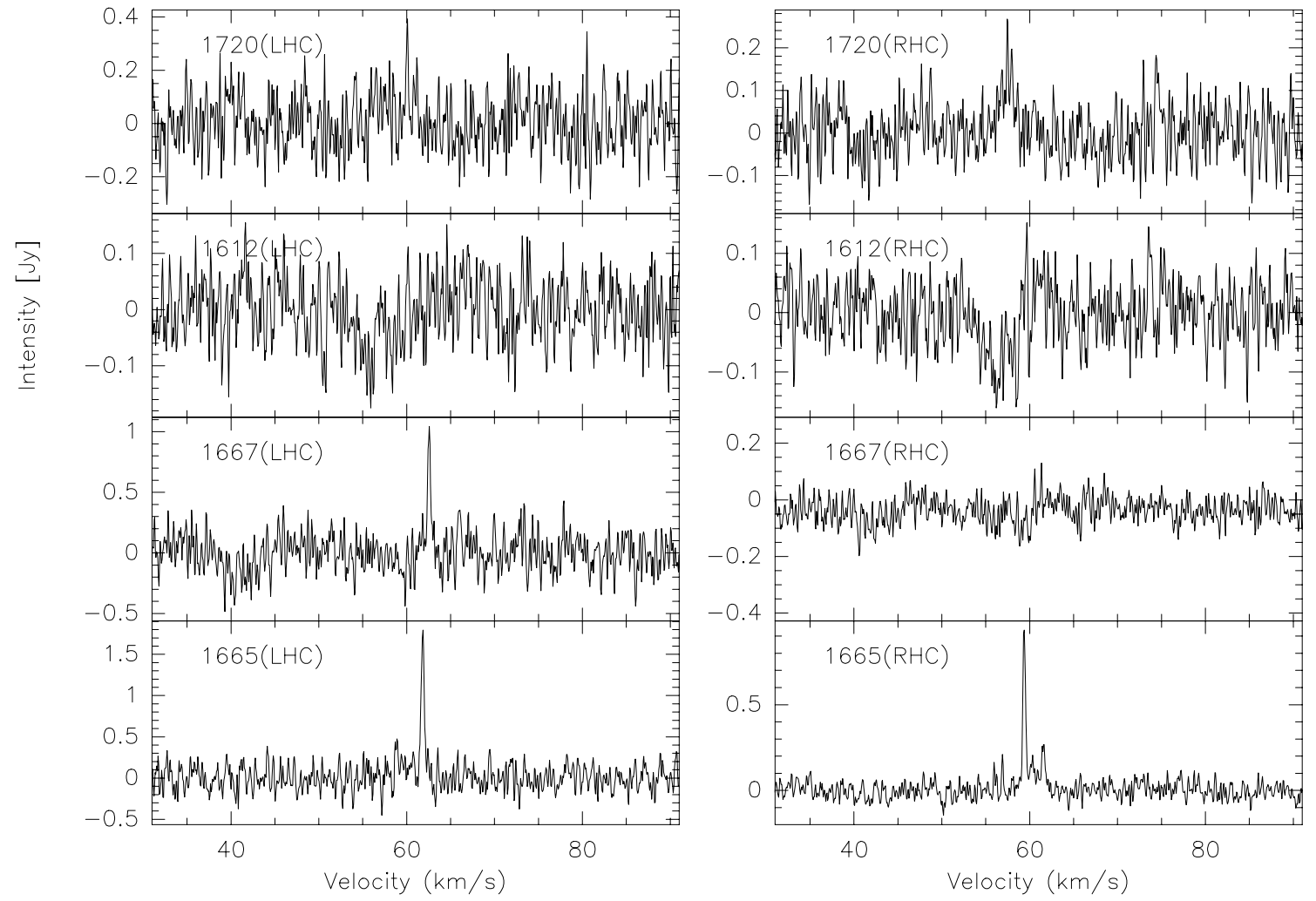

Fig. 19. continued. 
K. A. Edris et al.: OH maser survey towards HMPOs, Online Material p 14

IRAS18236-1205
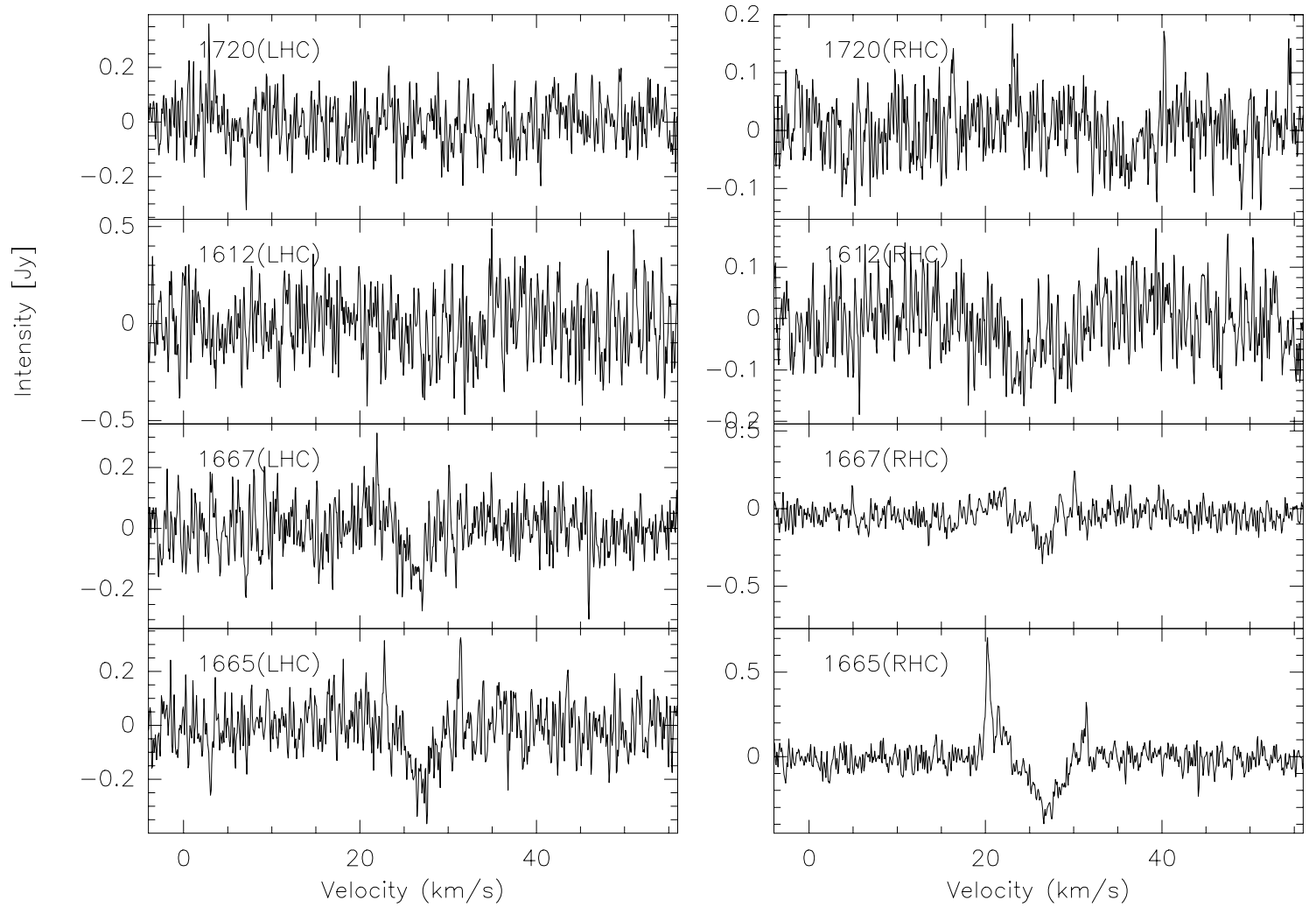

IRAS18264-1152
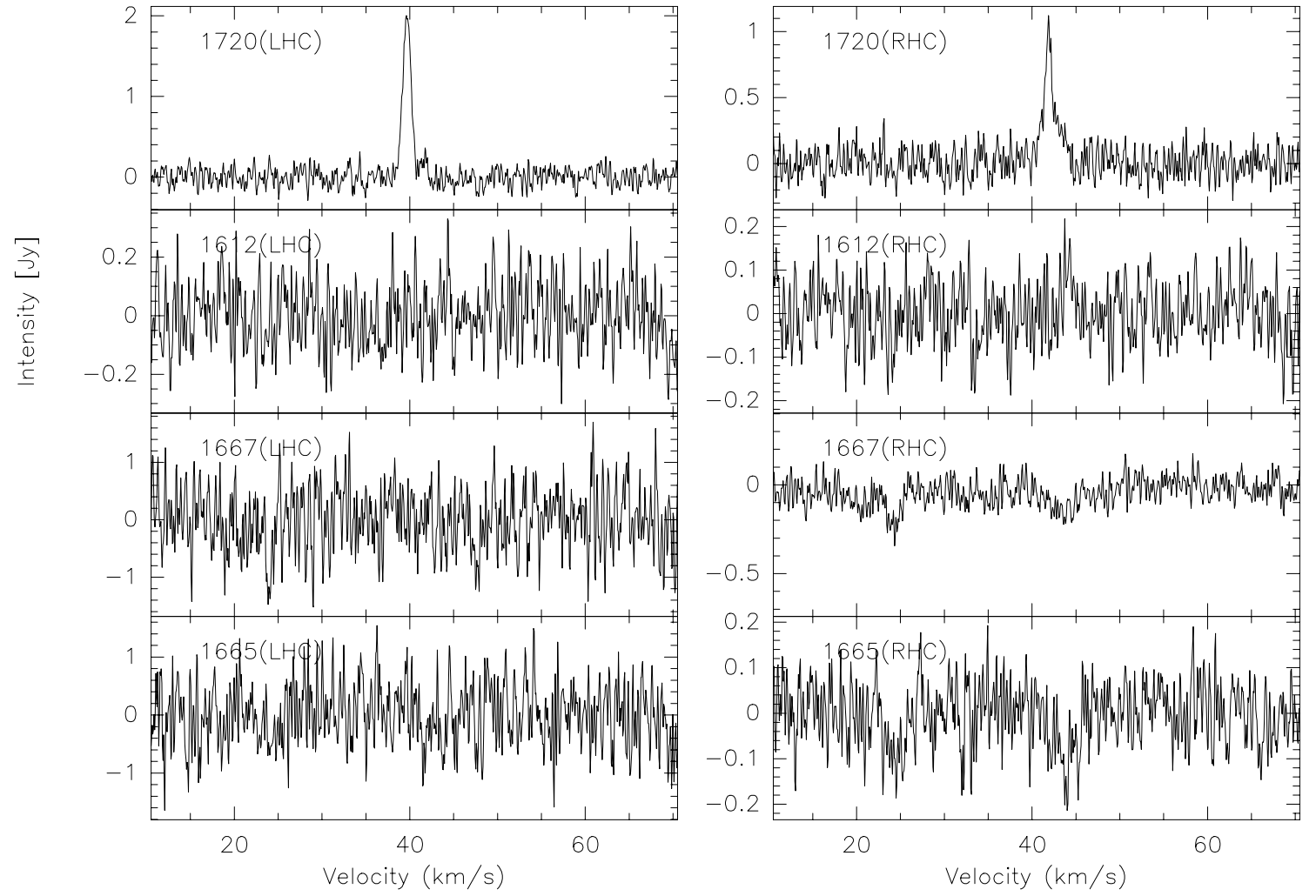

Fig. 19. continued. 
K. A. Edris et al.: OH maser survey towards HMPOs, Online Material p 15

IRAS18278-1009
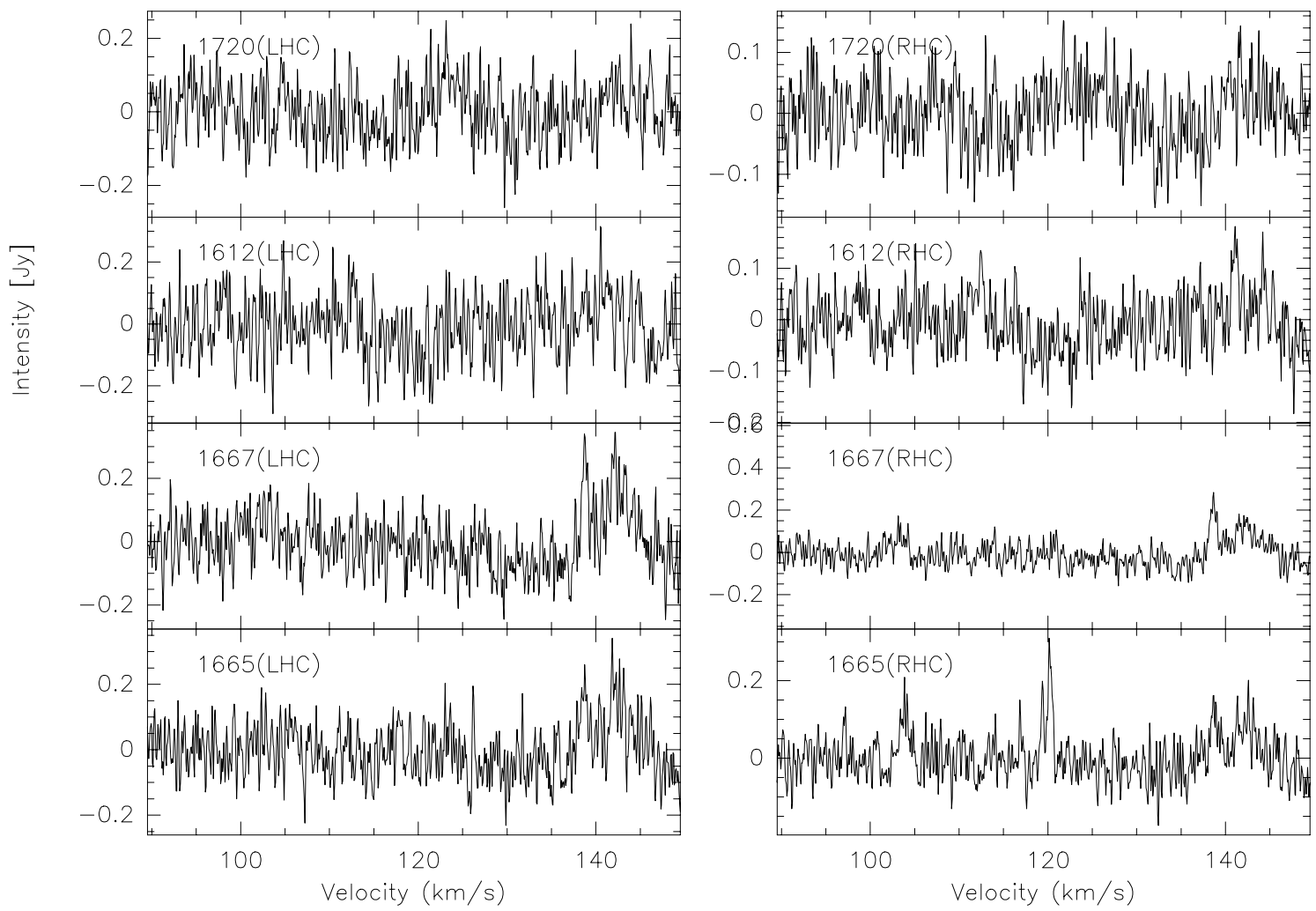

IRAS18290-0924
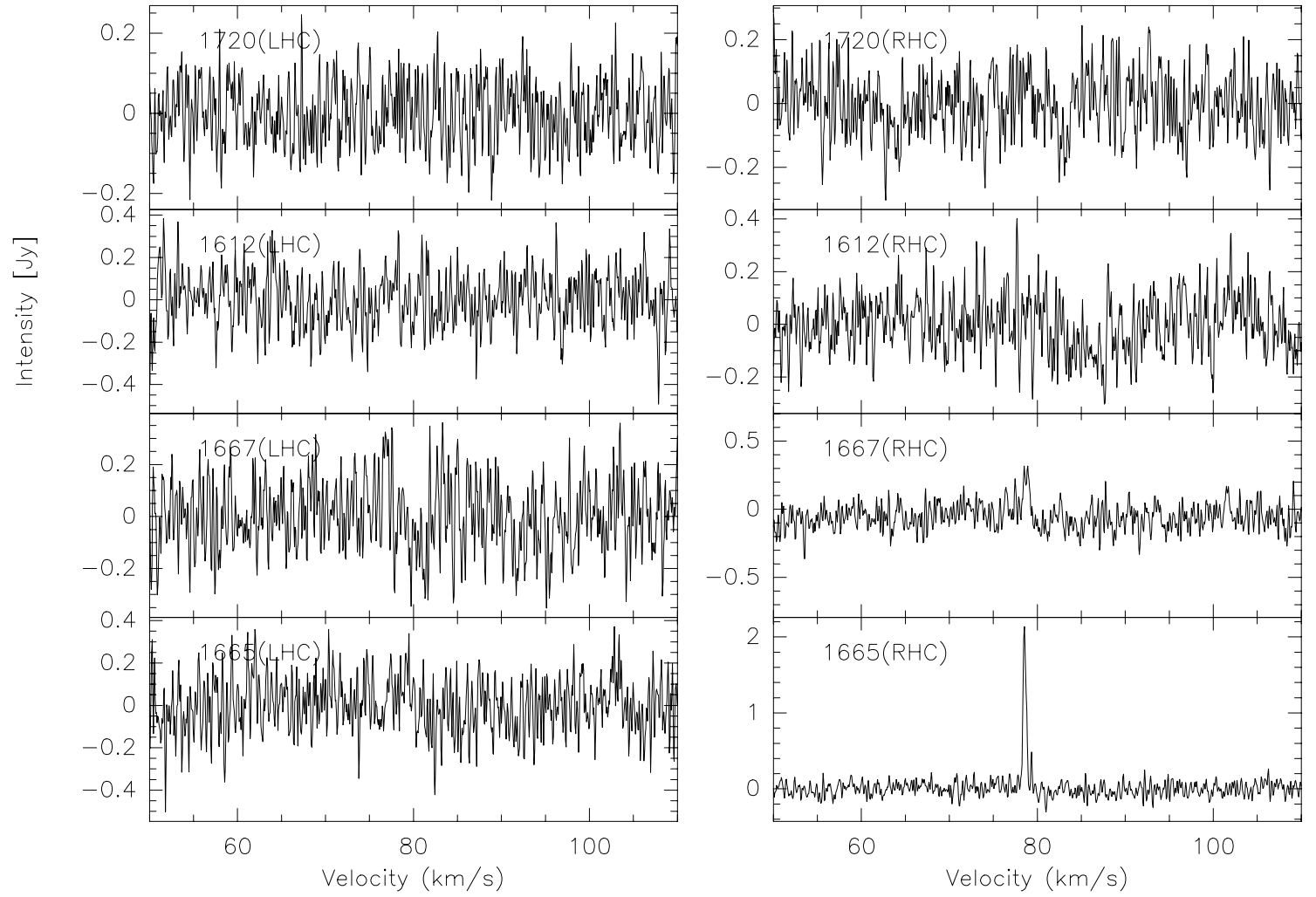

Fig. 19. continued. 
K. A. Edris et al.: OH maser survey towards HMPOs, Online Material p 16

IRAS18310-0825(R)
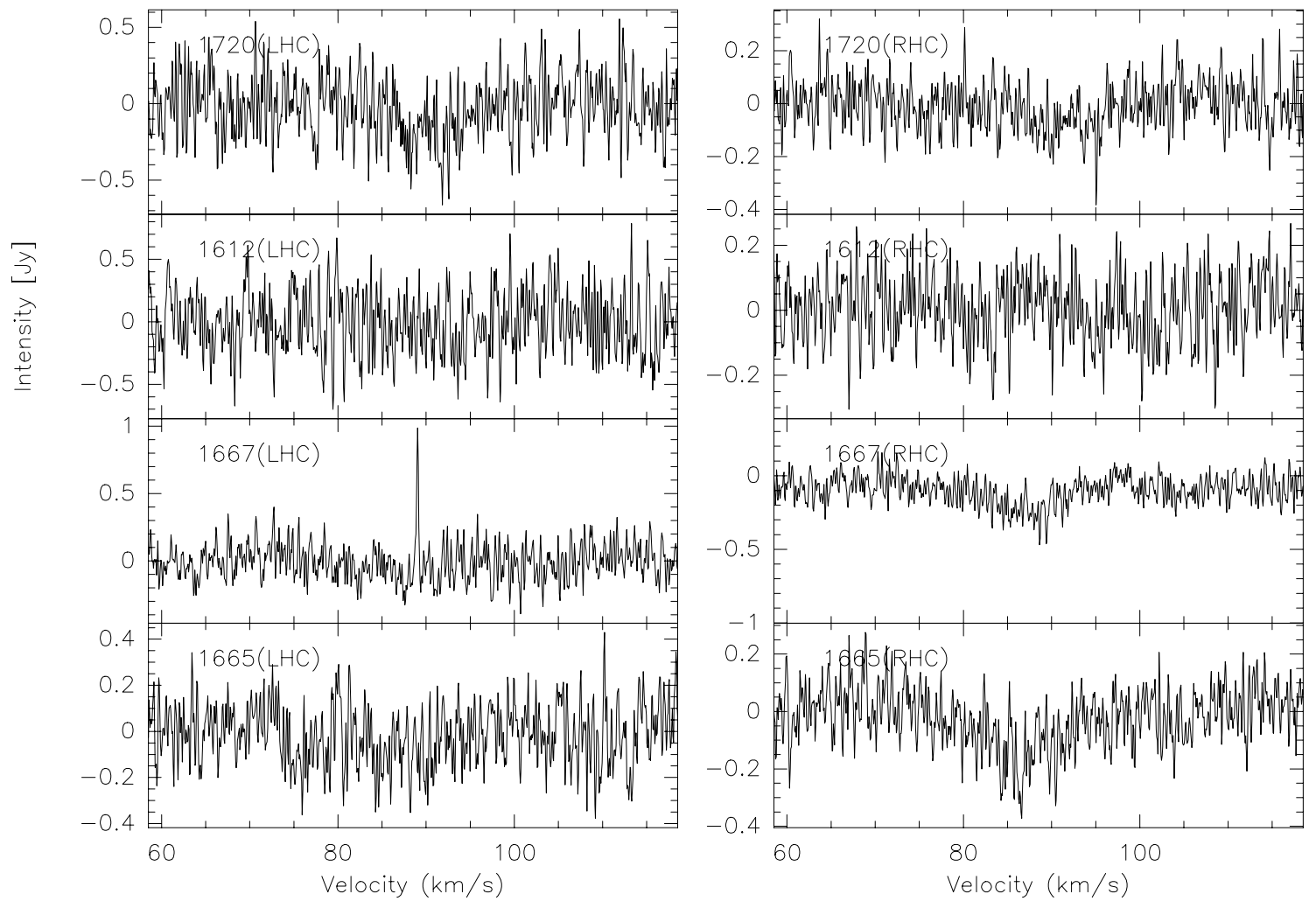

IRAS18316-0602
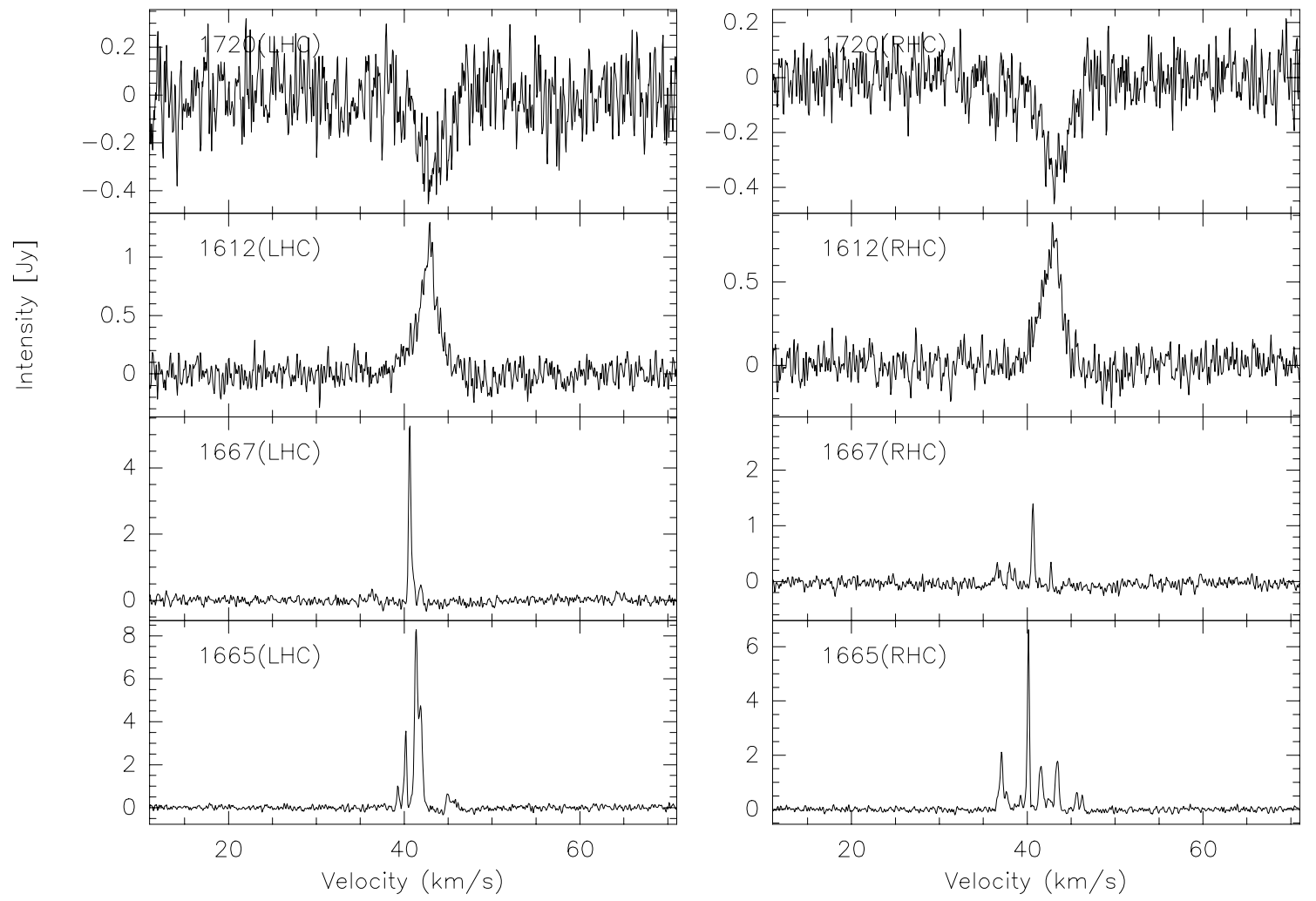

Fig. 19. continued. 
K. A. Edris et al.: OH maser survey towards HMPOs, Online Material p 17

IRAS18345-0641
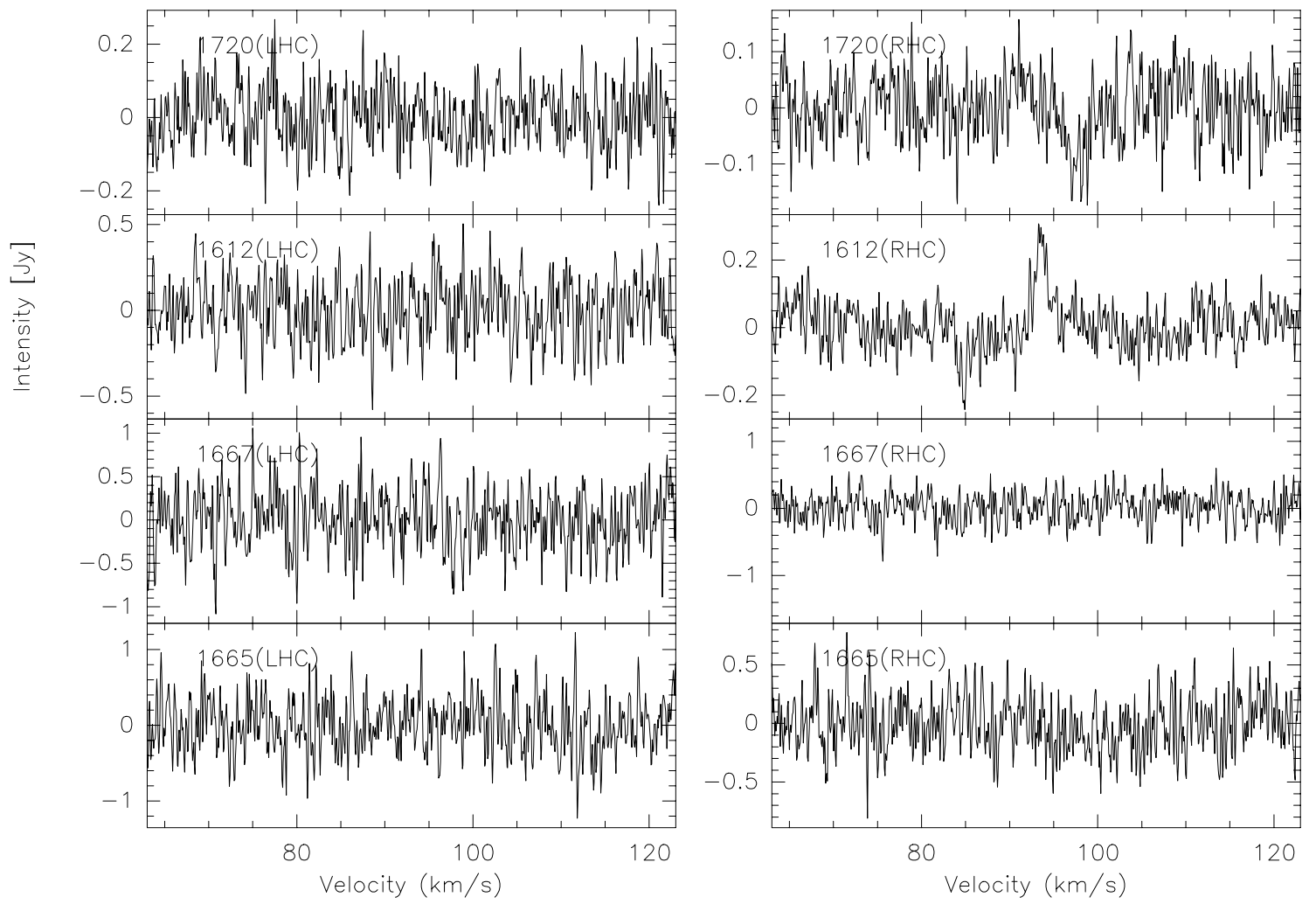

IRAS18360-0537
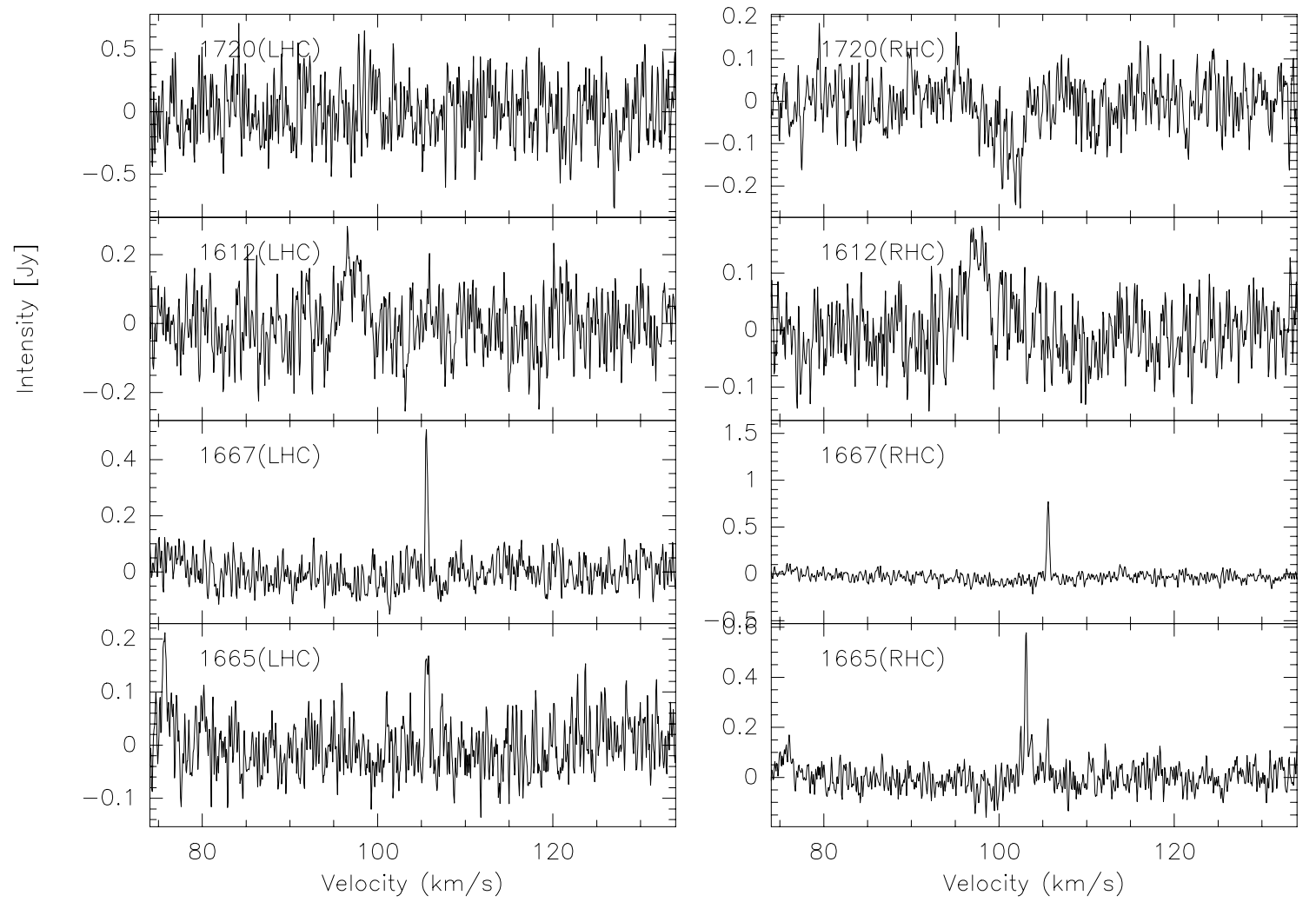

Fig. 19. continued. 
K. A. Edris et al.: OH maser survey towards HMPOs, Online Material p 18

IRAS18385-0512
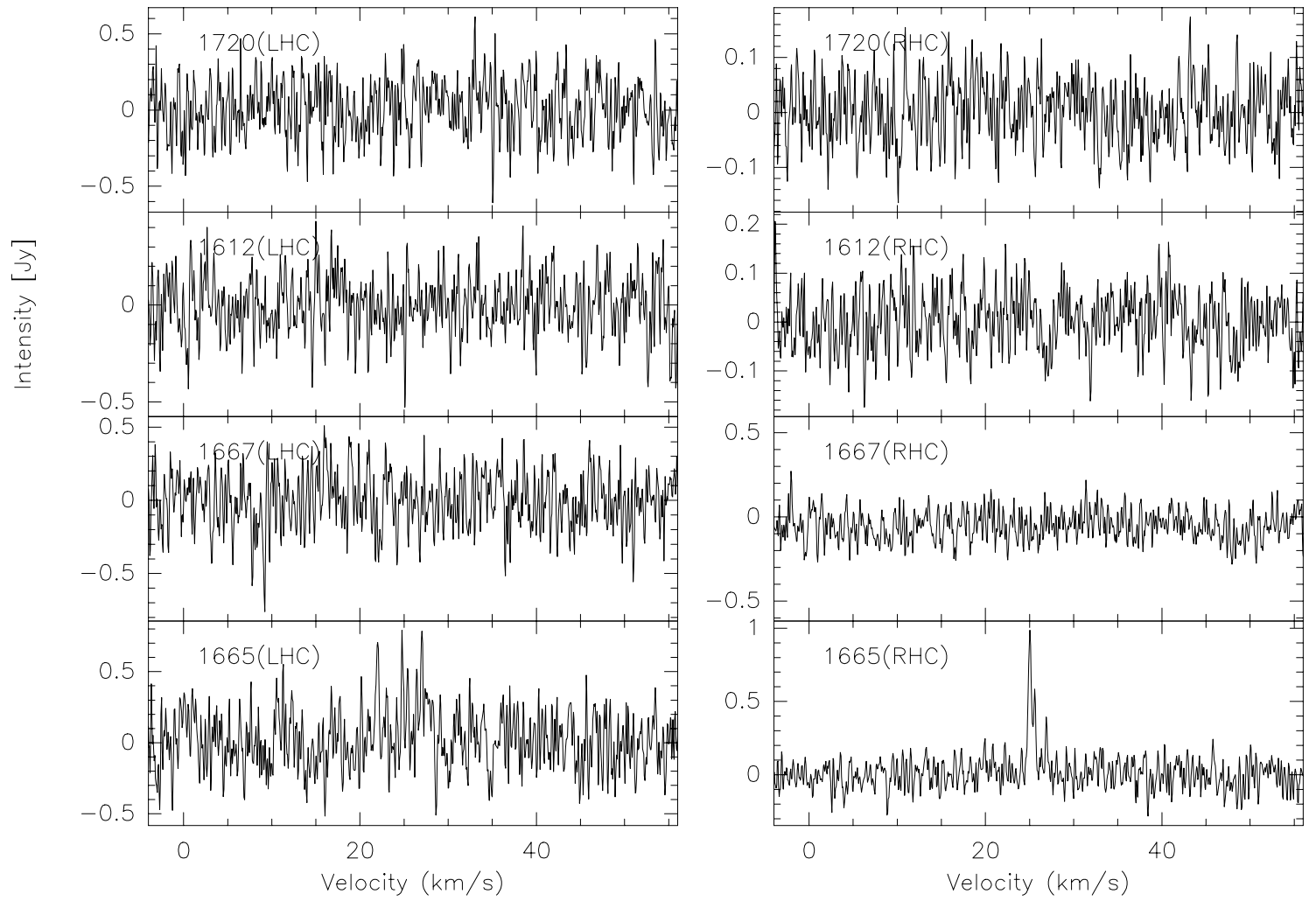

IRAS18440-0148
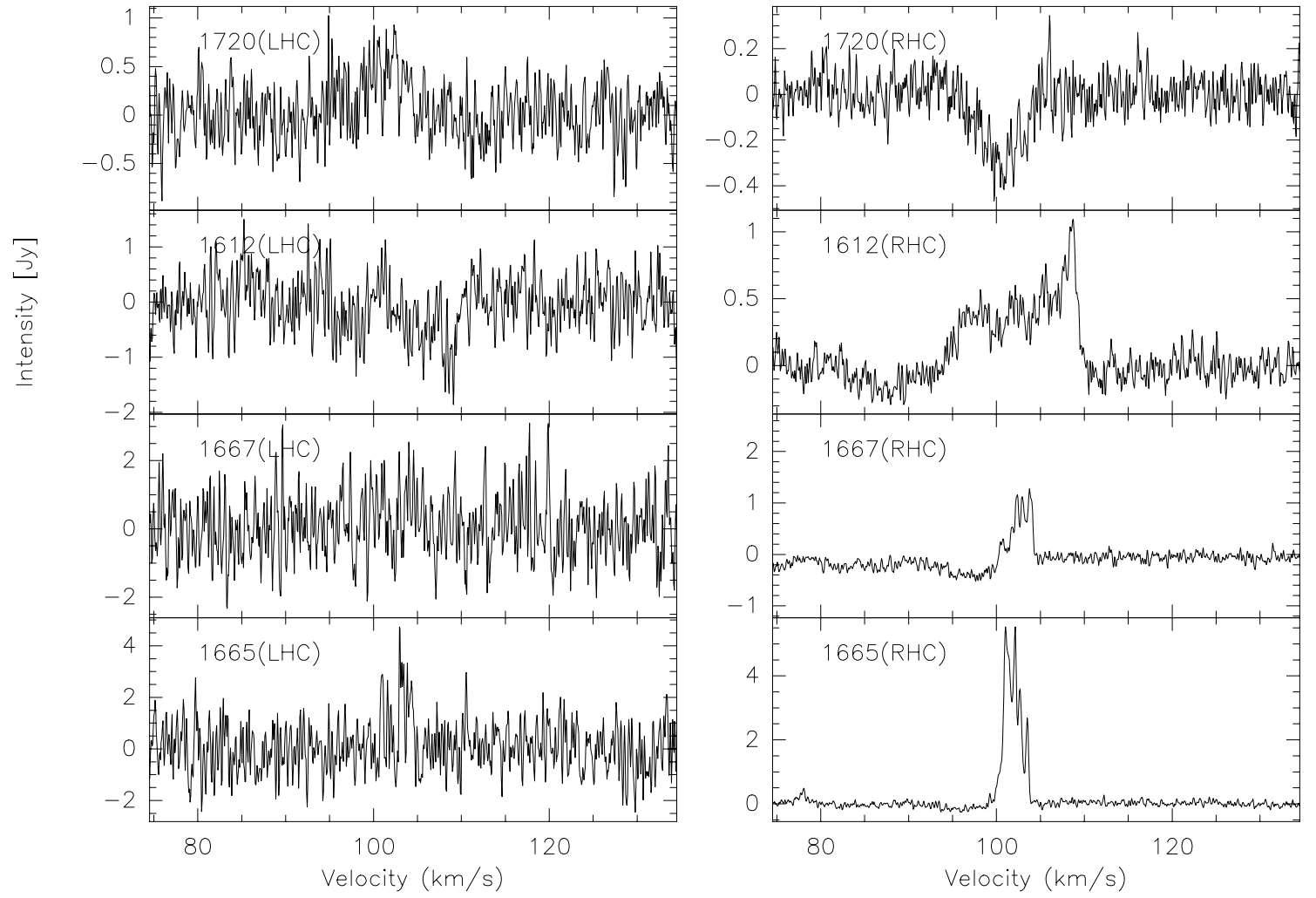

Fig. 19. continued. 
K. A. Edris et al.: OH maser survey towards HMPOs, Online Material p 19

IRAS18454-0158
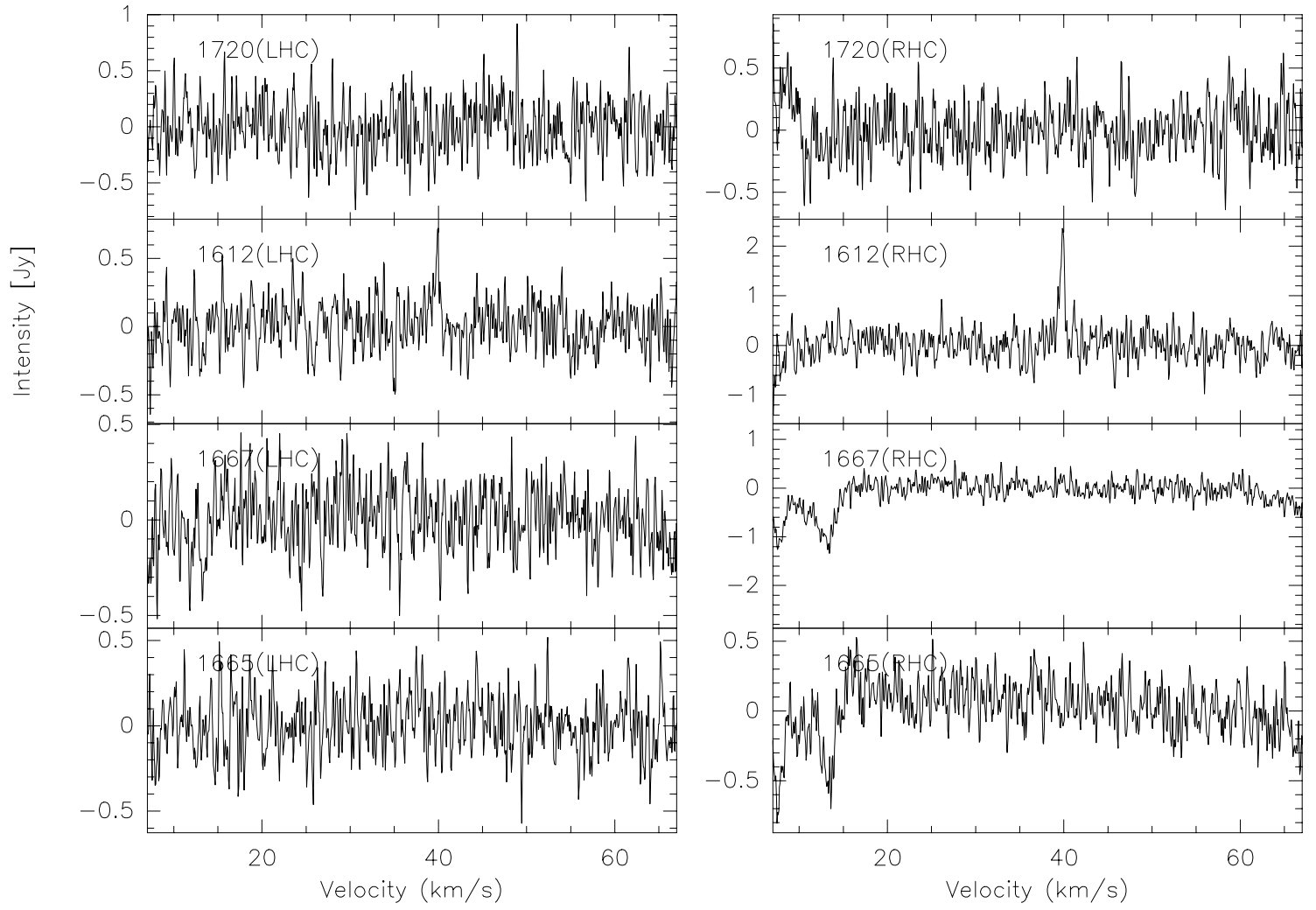

IRAS18463+0052
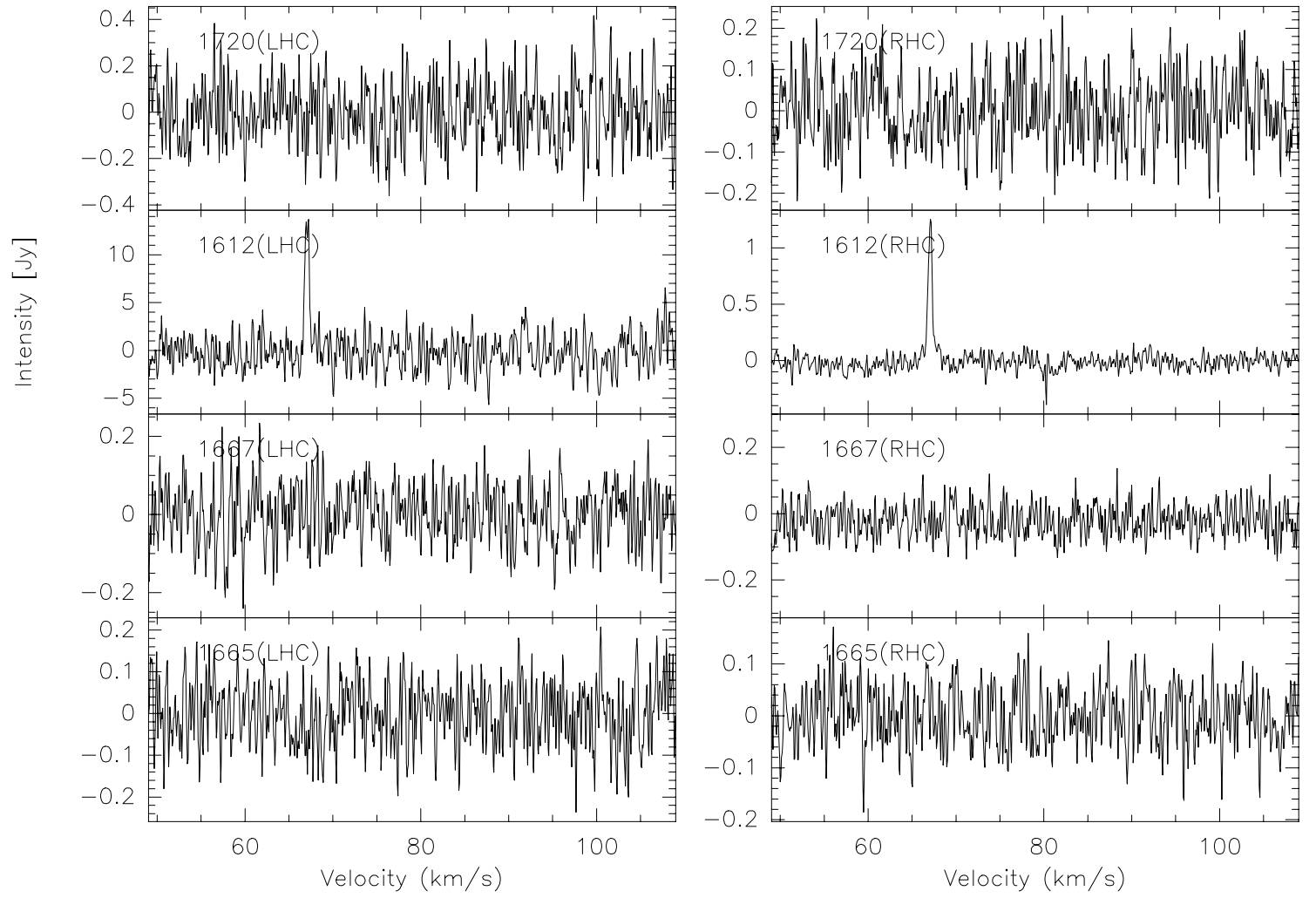

Fig. 19. continued. 
K. A. Edris et al.: OH maser survey towards HMPOs, Online Material p 20

IRAS18488+0000
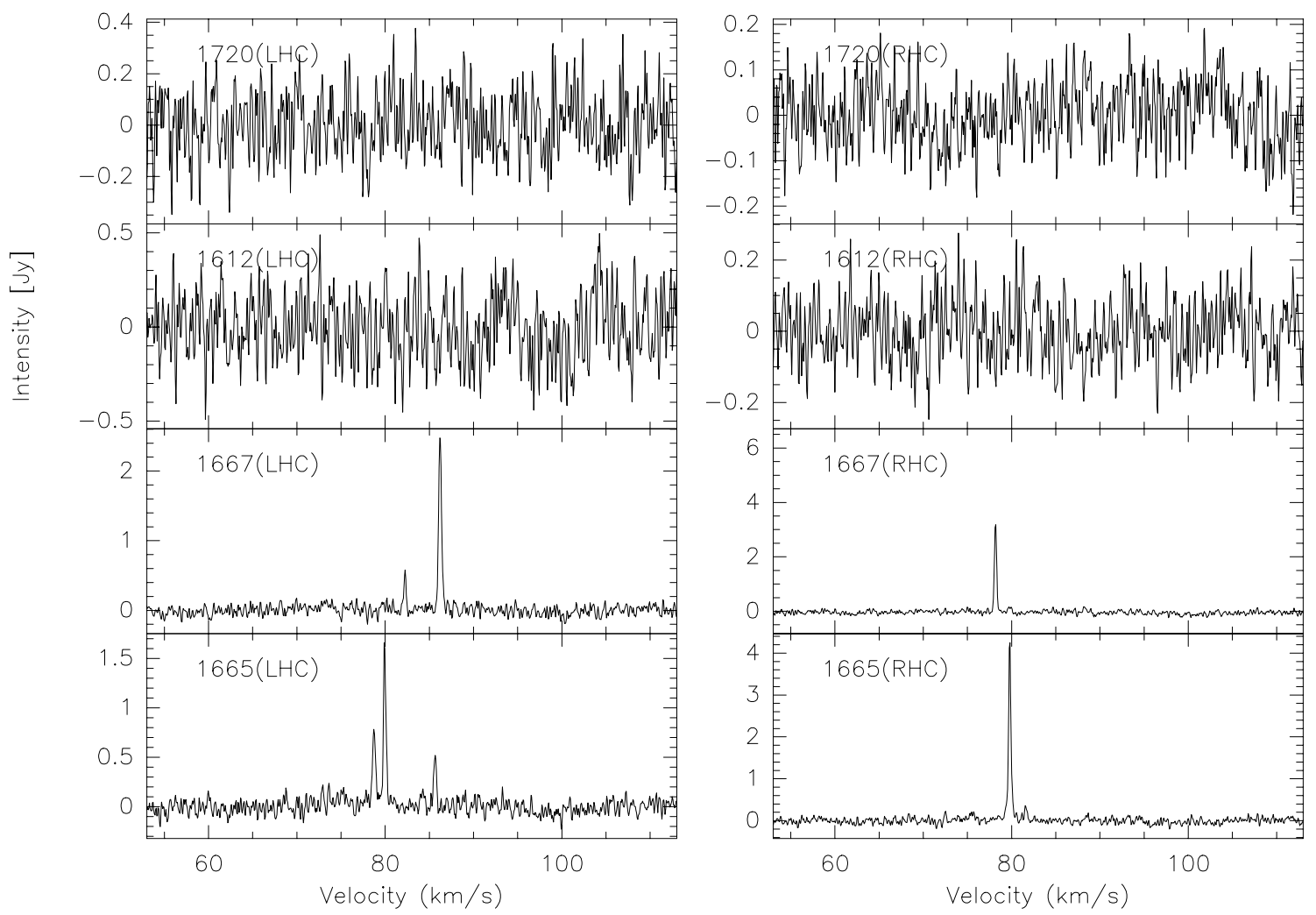

IRAS18507+0121
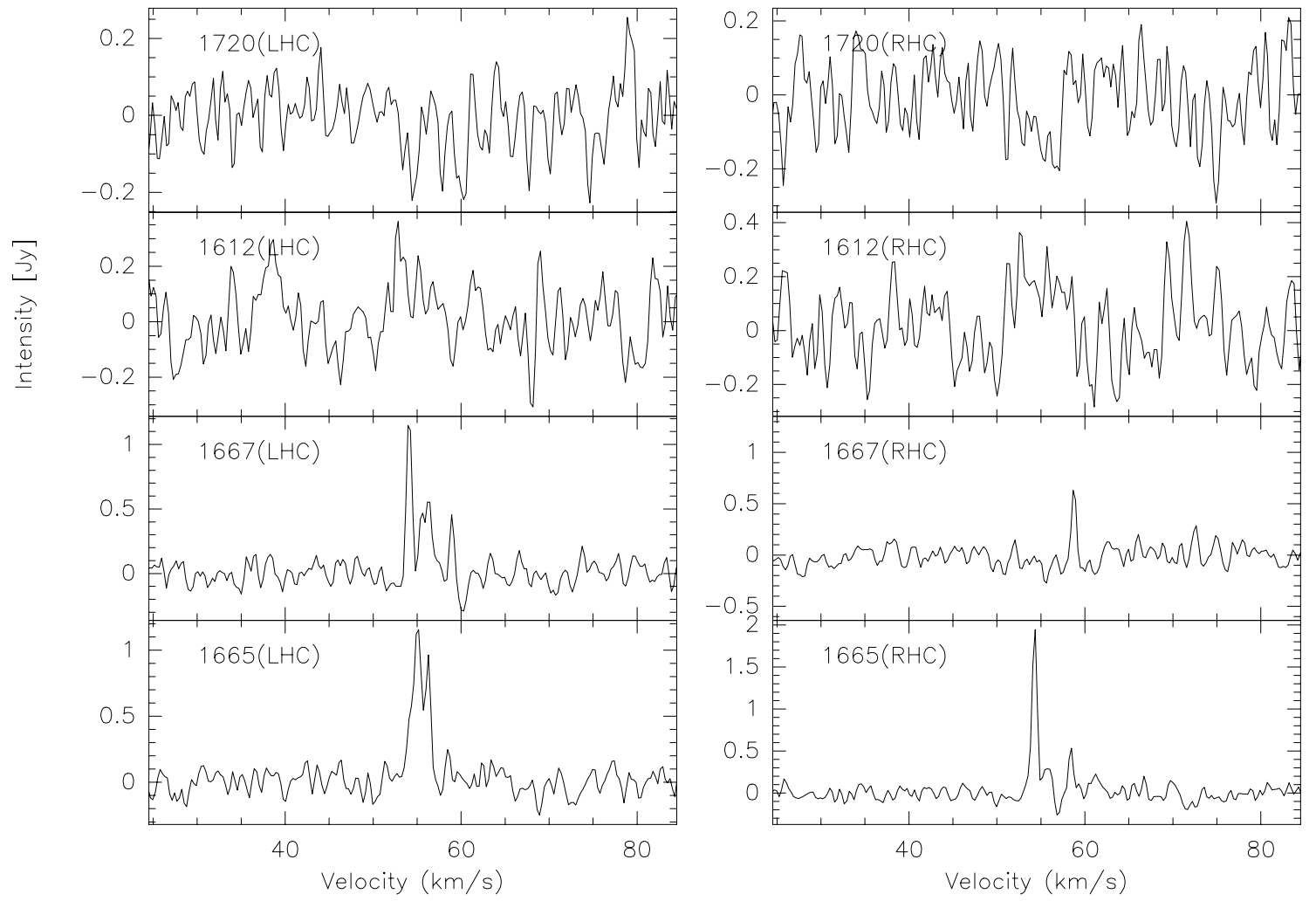

Fig. 19. continued. 
K. A. Edris et al.: OH maser survey towards HMPOs, Online Material p 21

IRAS18527+0301
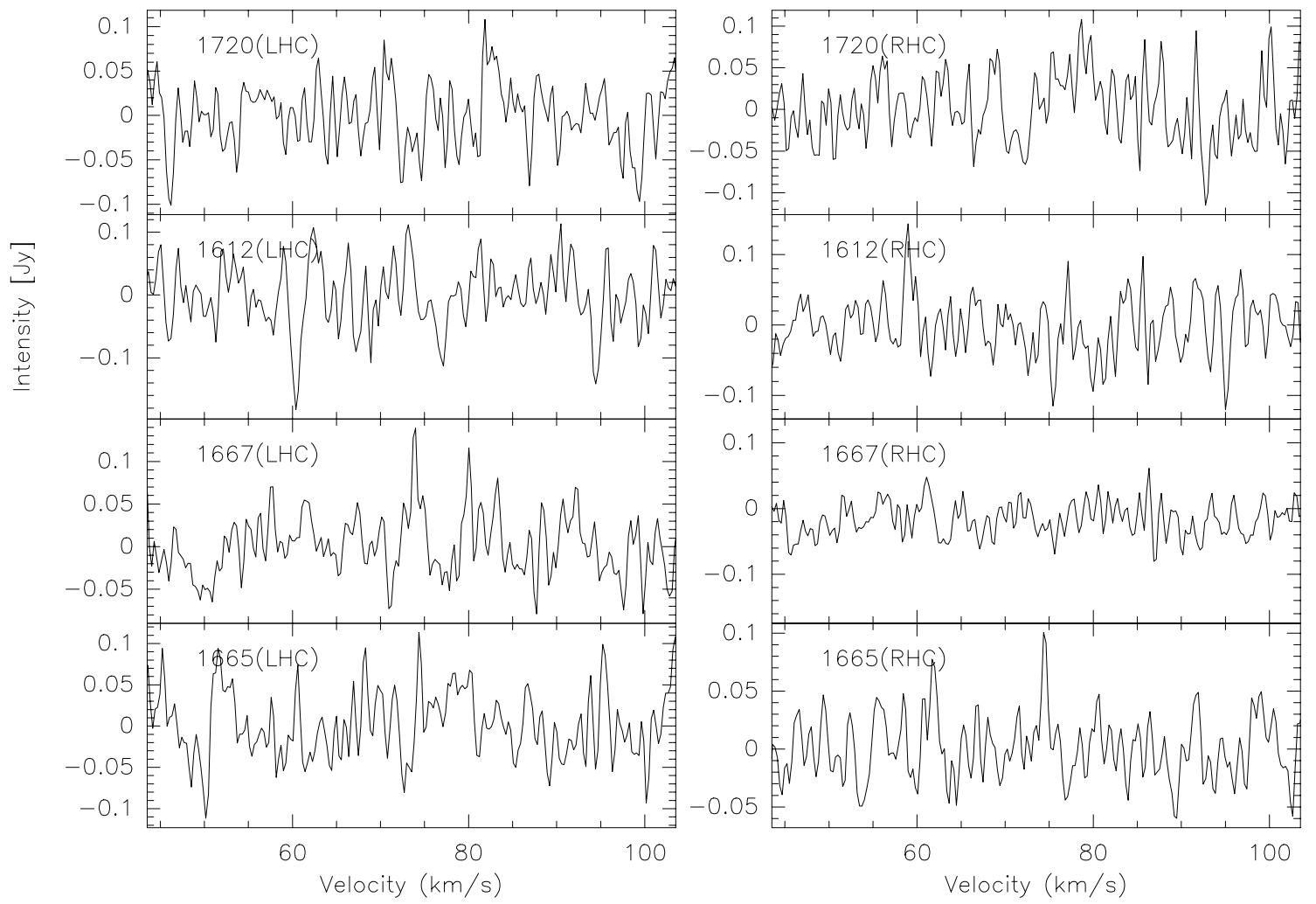

IRAS18553+0414
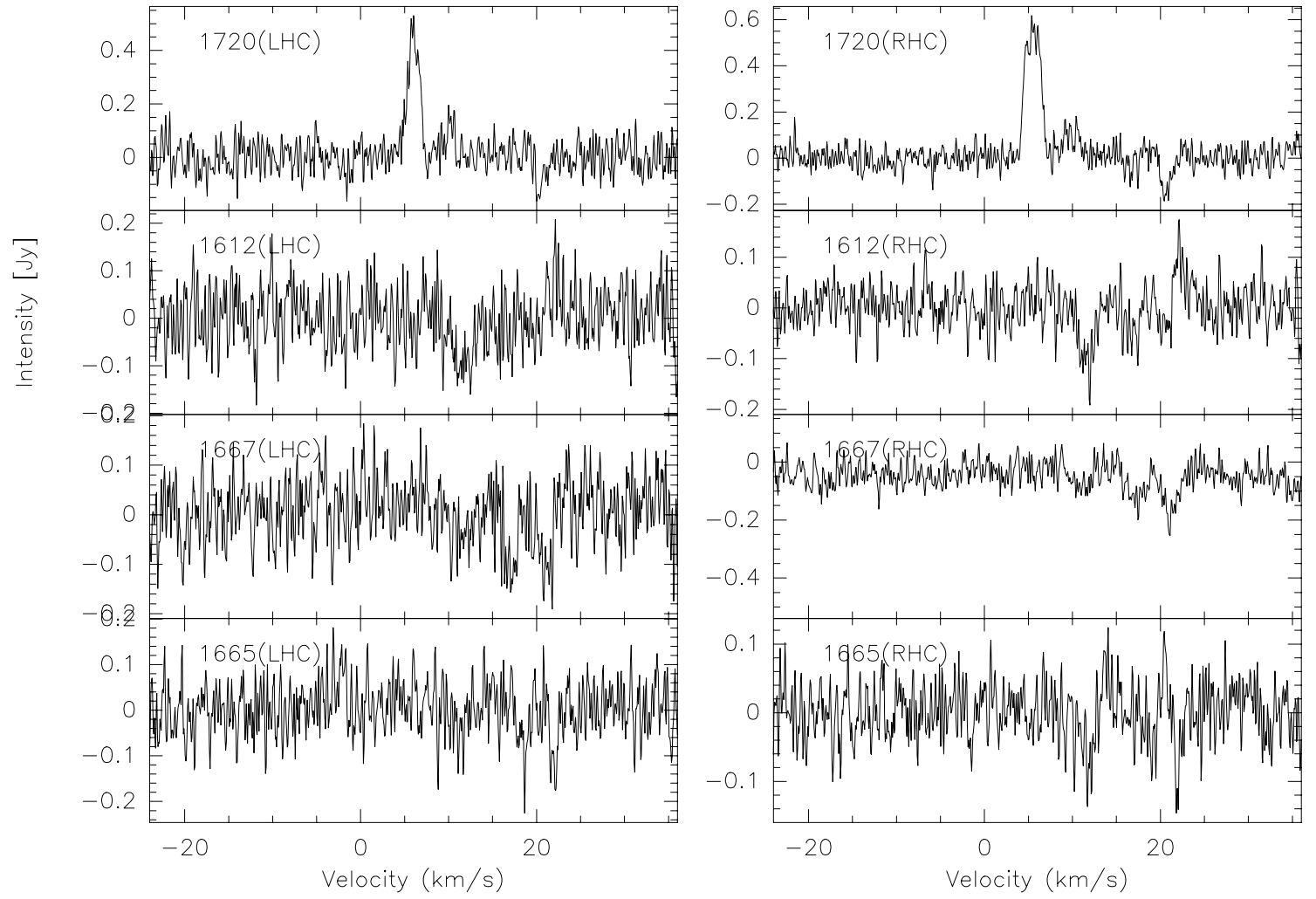

Fig. 19. continued. 
K. A. Edris et al.: OH maser survey towards HMPOs, Online Material p 22

IRAS18566+0408
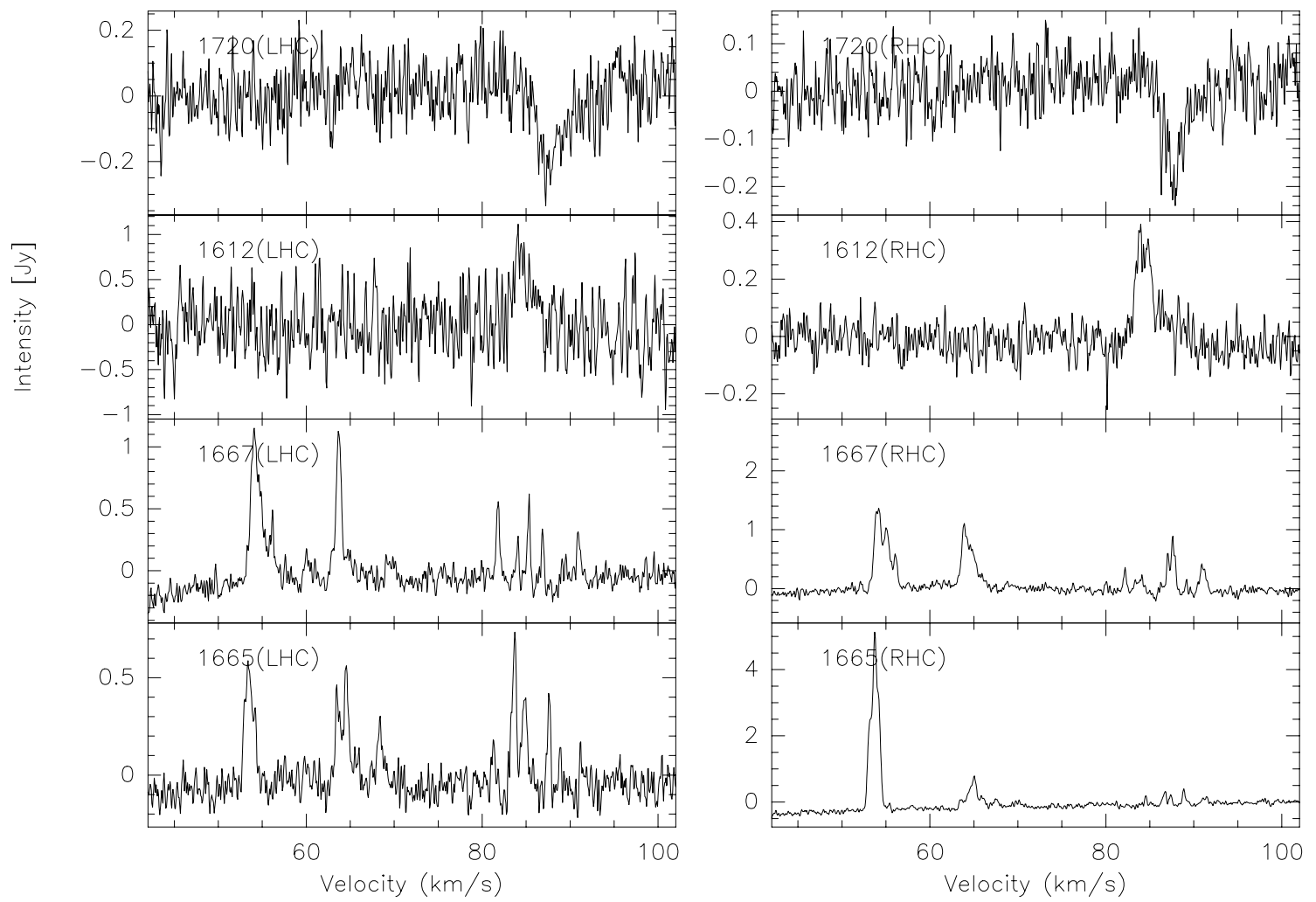

IRAS19035+0641
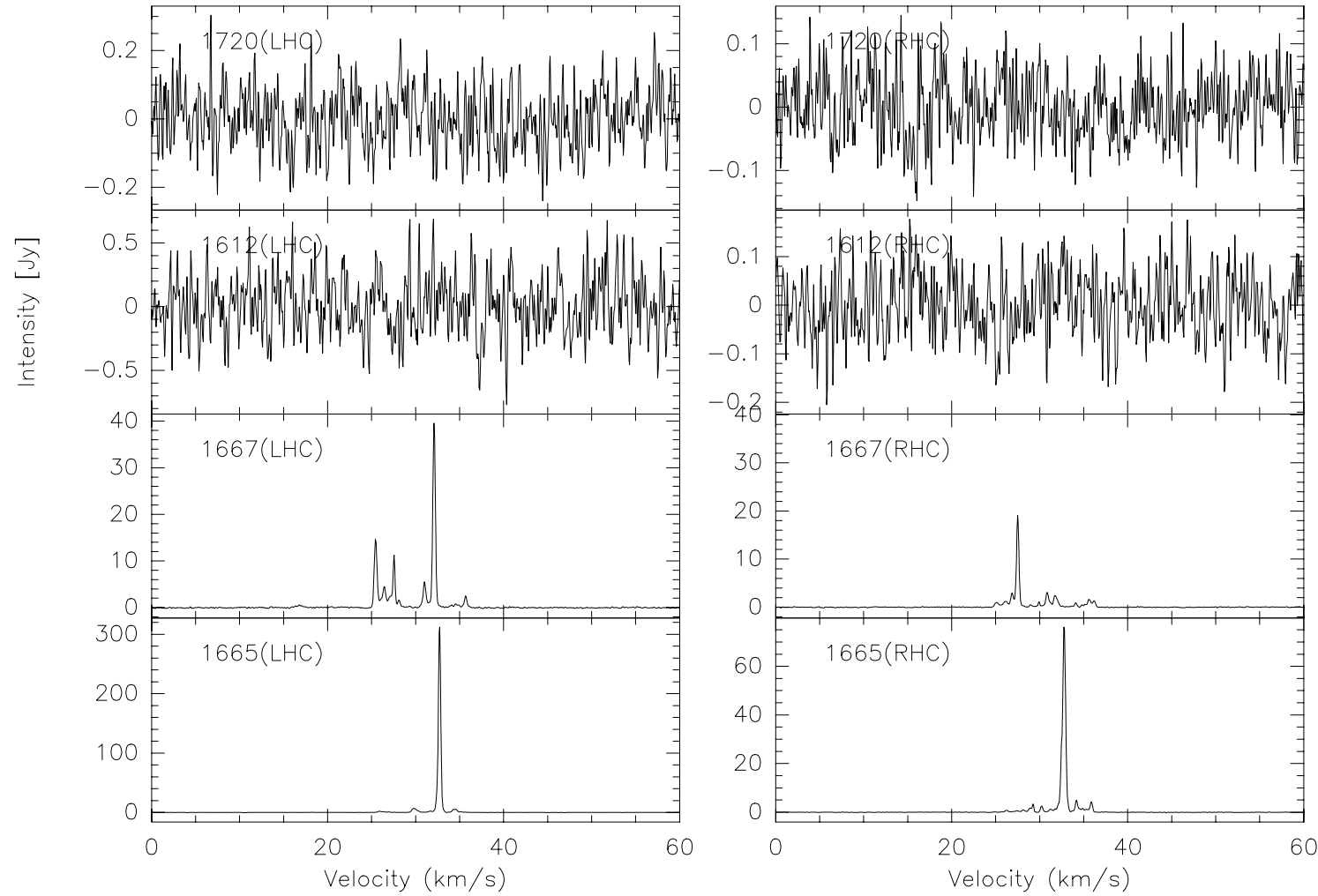

Fig. 19. continued. 
K. A. Edris et al.: OH maser survey towards HMPOs, Online Material p 23

IRAS19092+0841
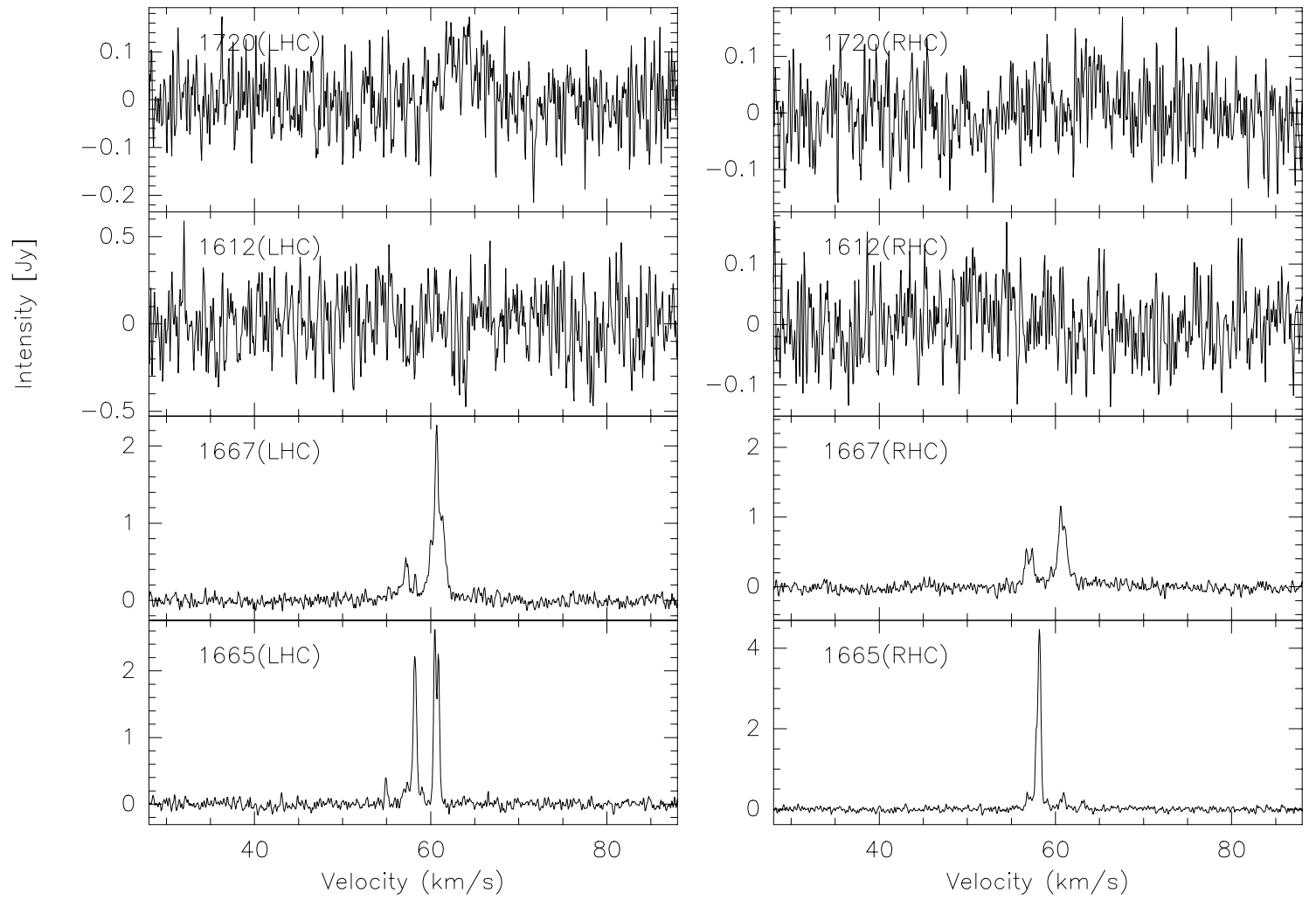

IRAS19118+0841
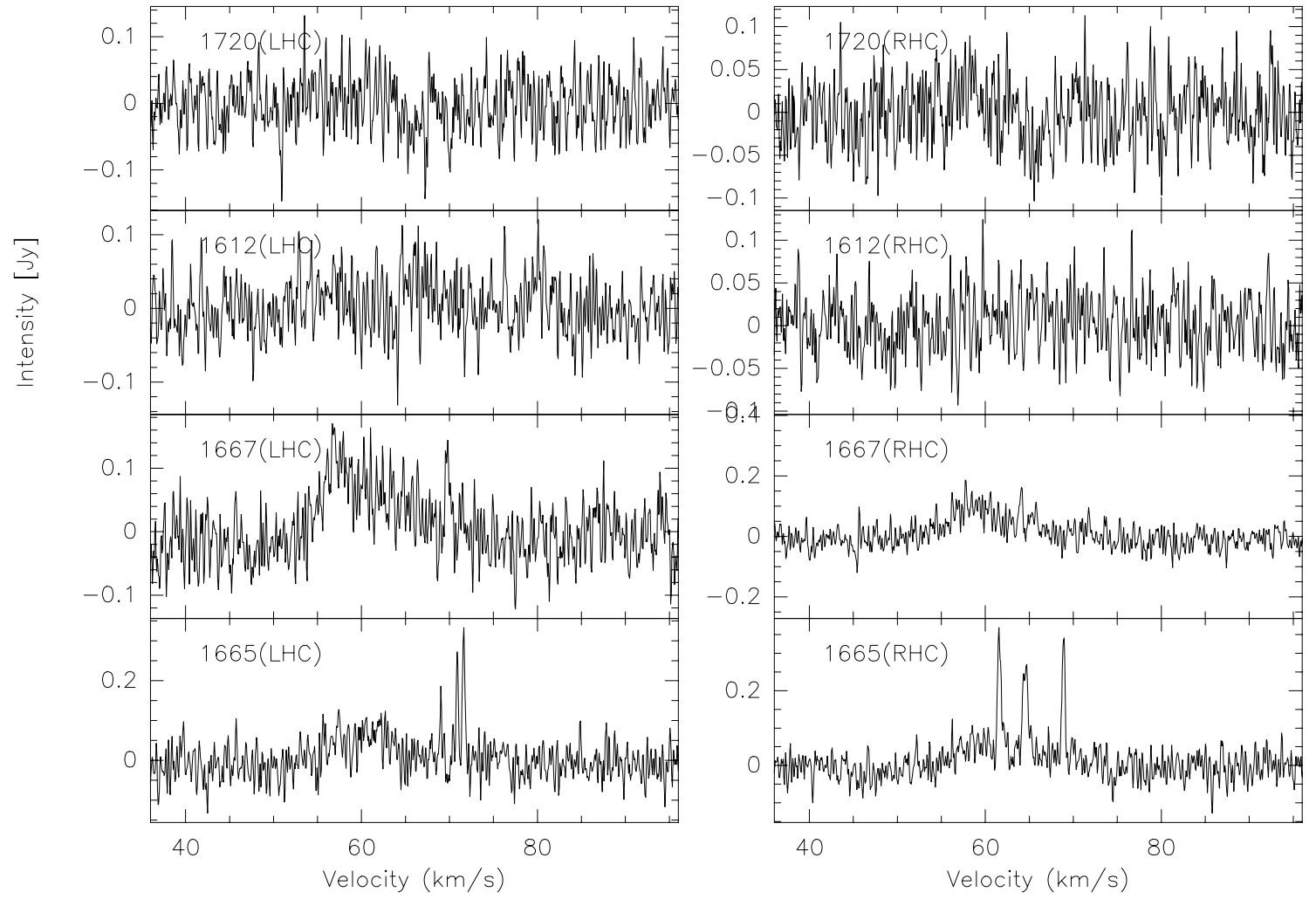

Fig. 19. continued. 
K. A. Edris et al.: OH maser survey towards HMPOs, Online Material p 24

IRAS19217+1651
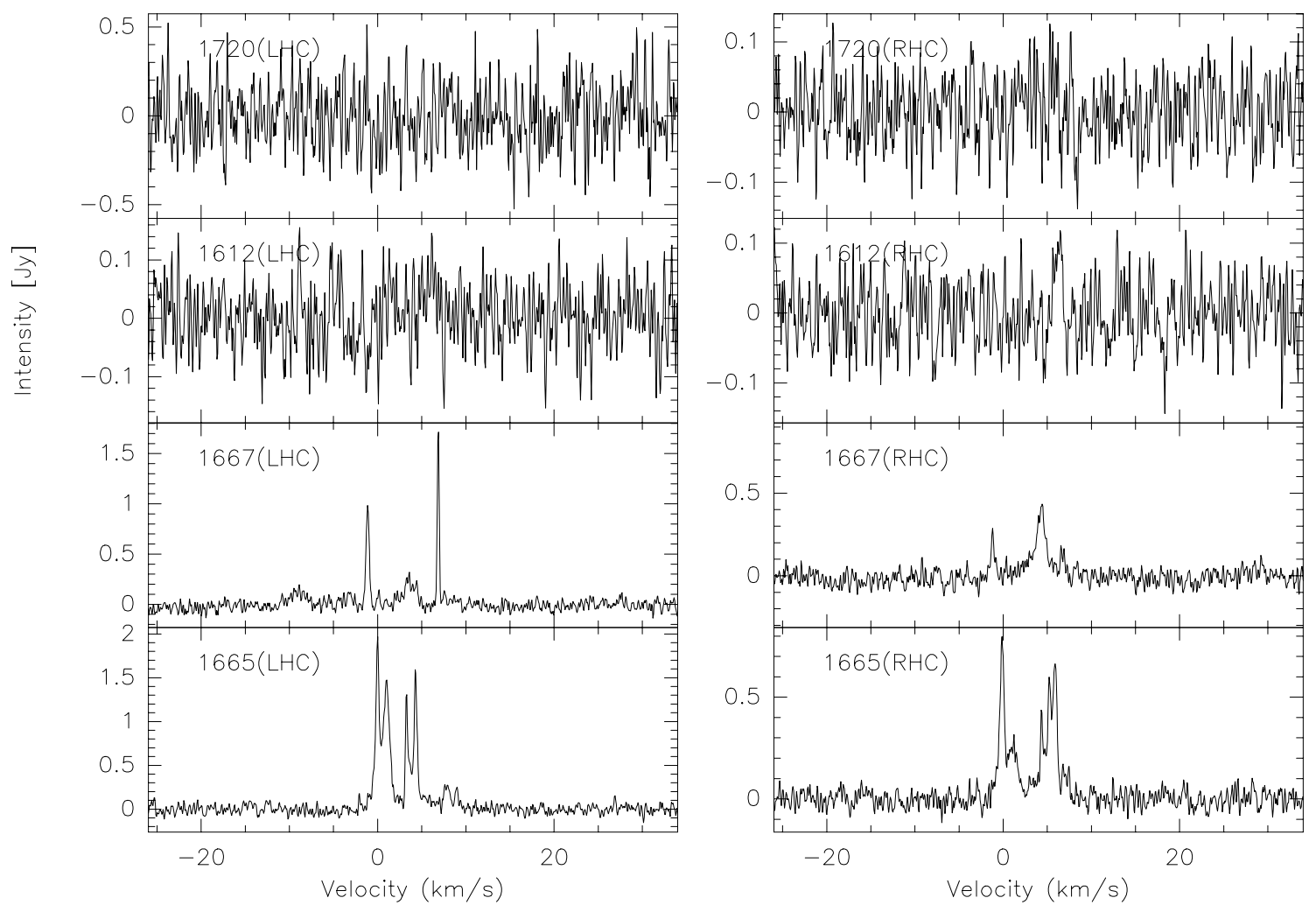

IRAS19220+1432
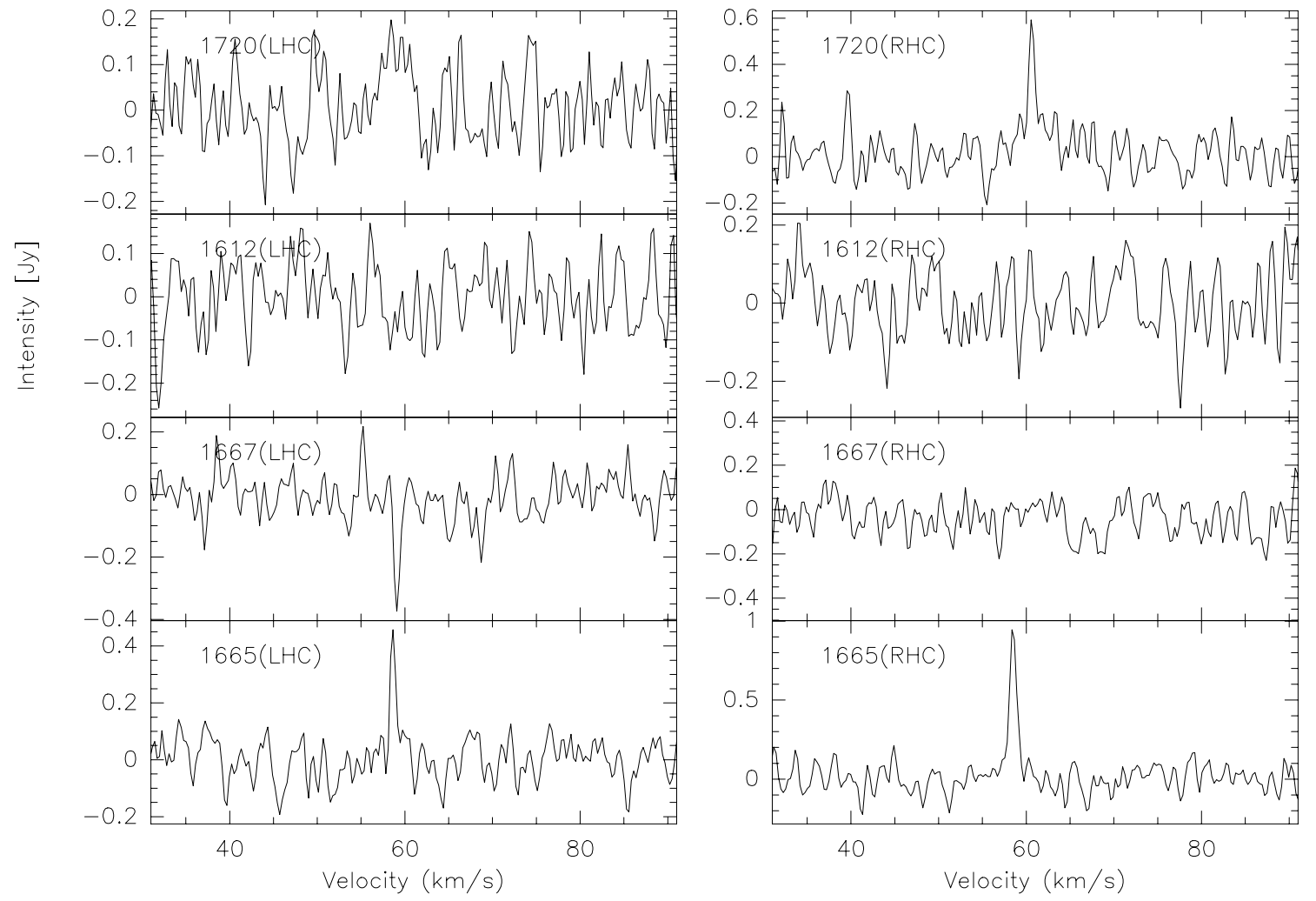

Fig. 19. continued. 
K. A. Edris et al.: OH maser survey towards HMPOs, Online Material p 25

IRAS19374+2352
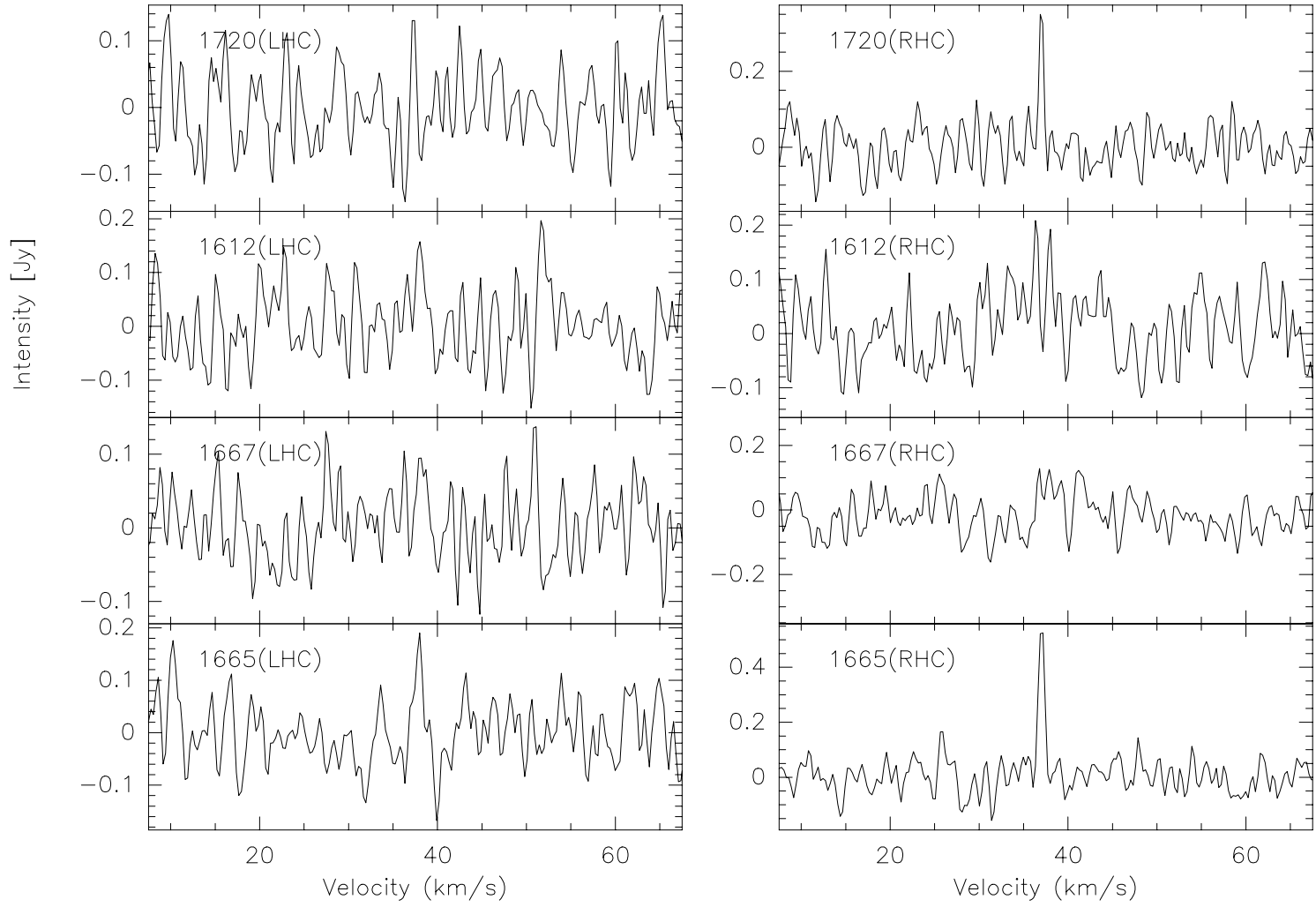

IRAS19388+2357
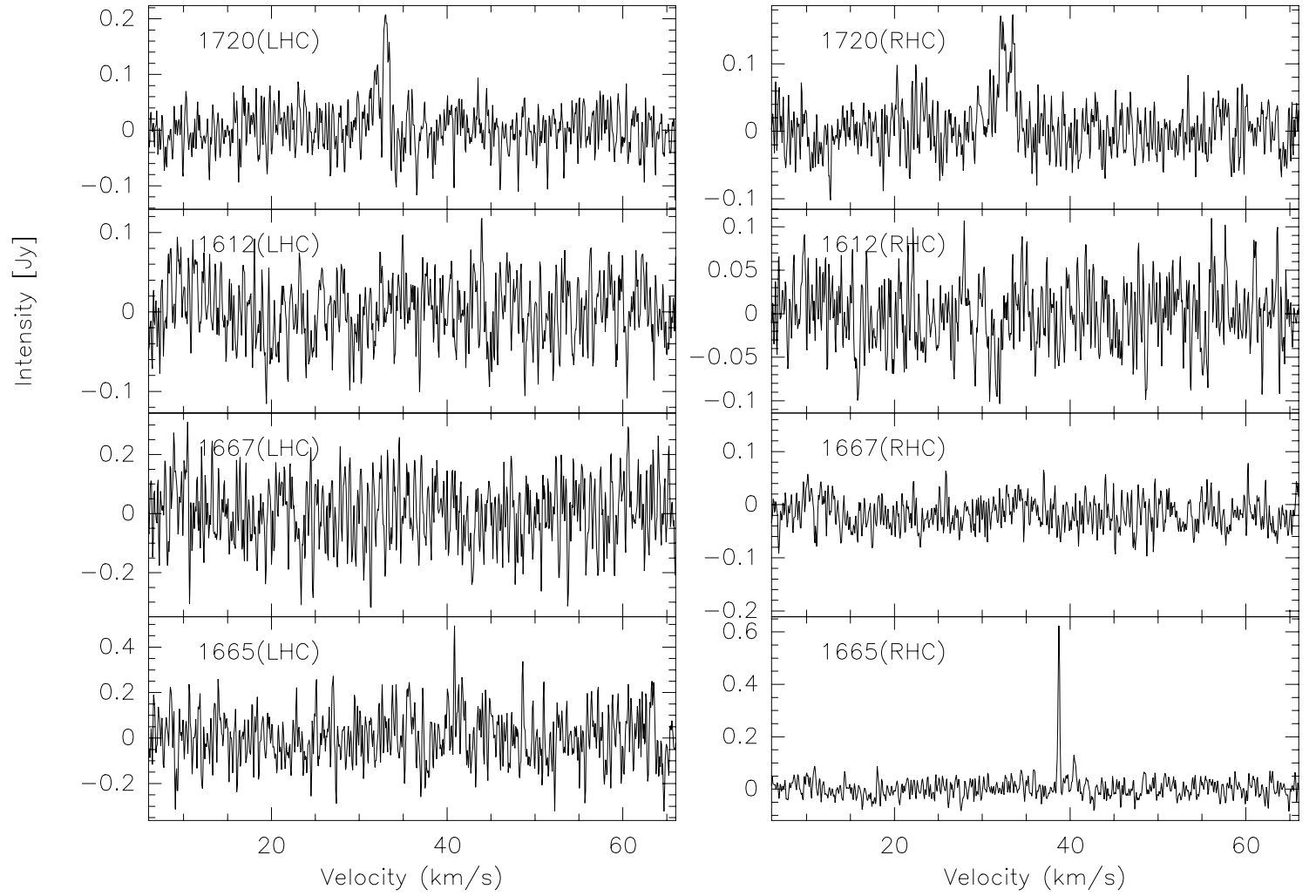

Fig. 19. continued. 
K. A. Edris et al.: OH maser survey towards HMPOs, Online Material p 26

IRAS19410+2336
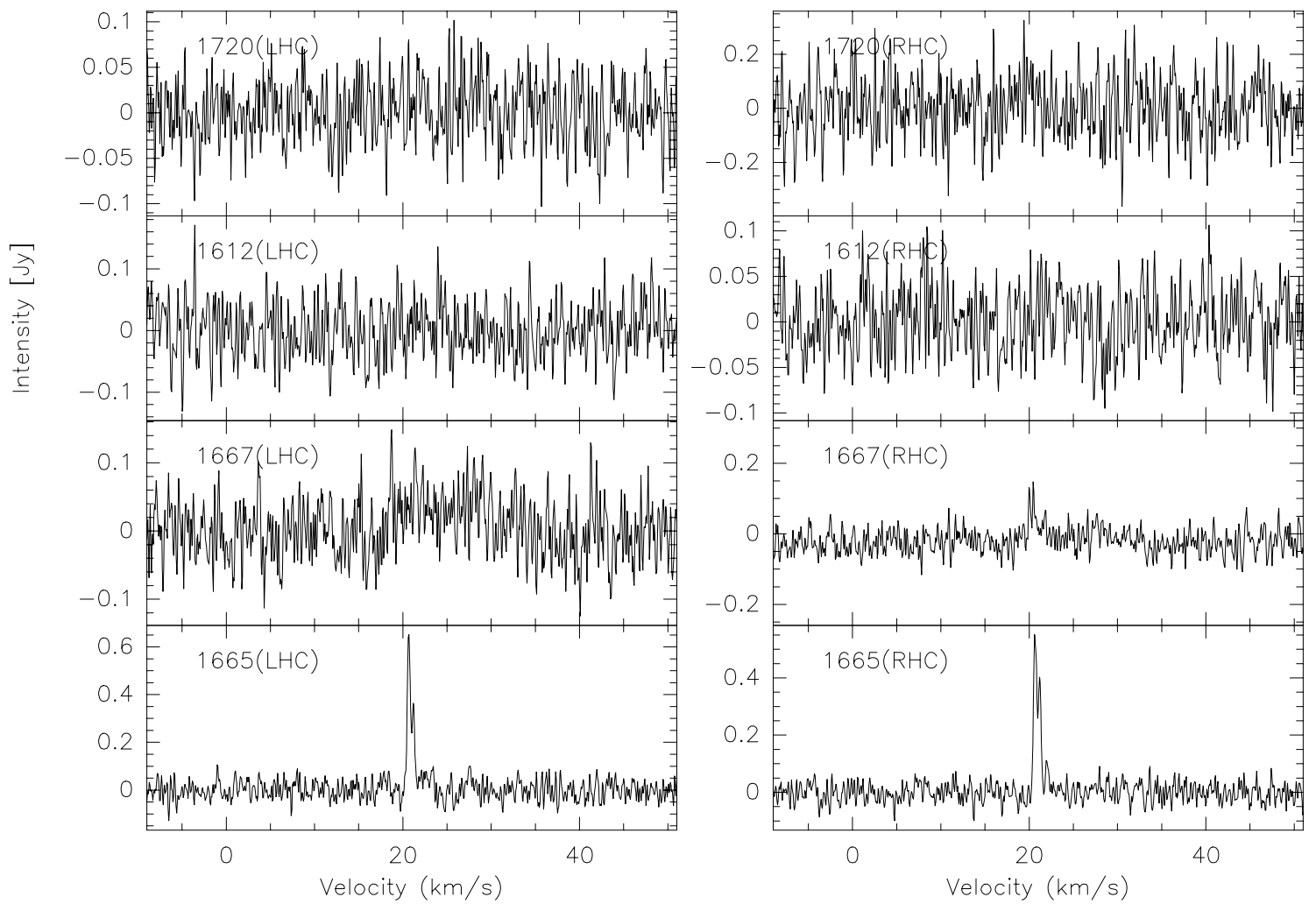

IRAS20062+3550
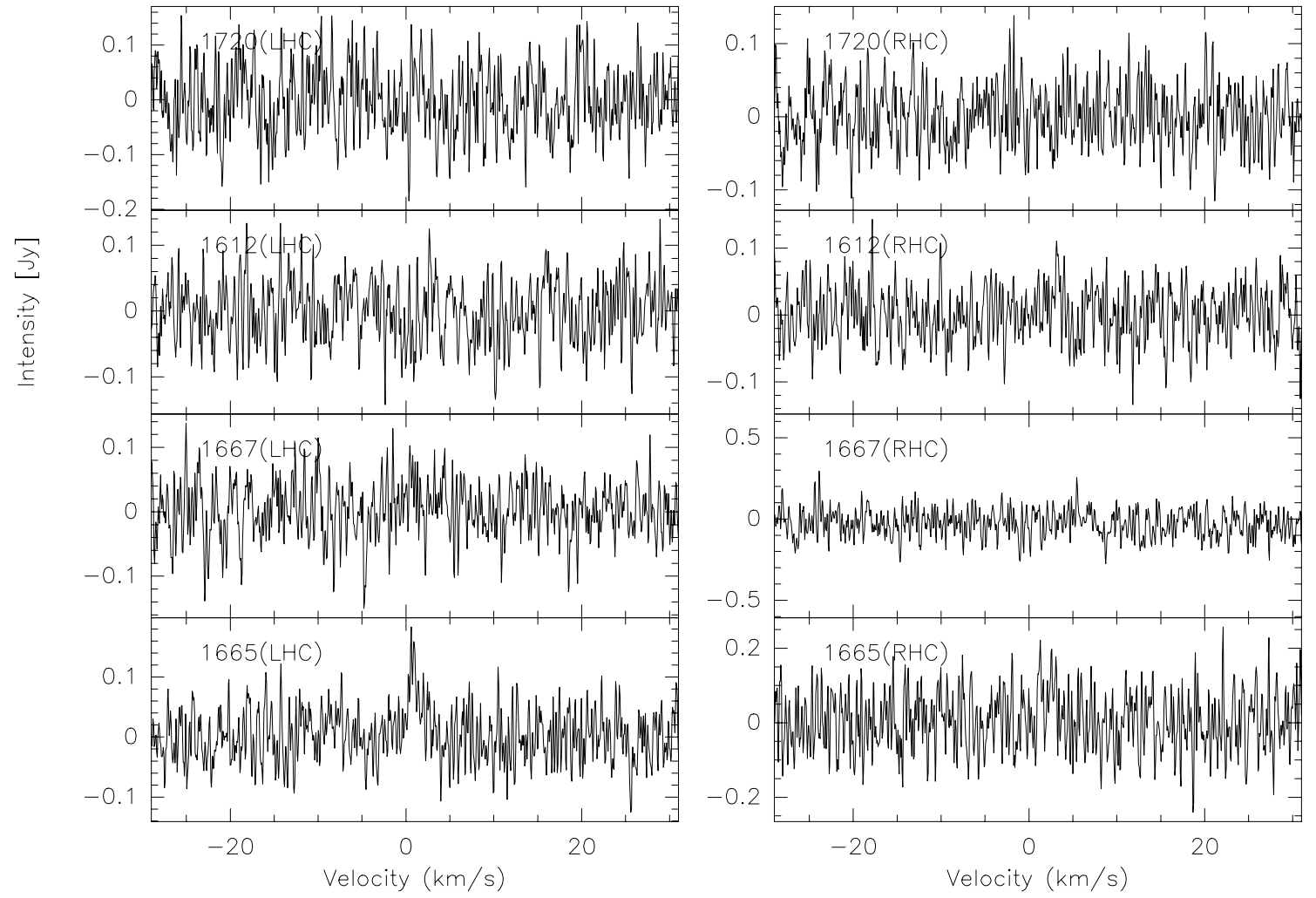

Fig. 19. continued. 
K. A. Edris et al.: OH maser survey towards HMPOs, Online Material p 27

IRAS20126+4104
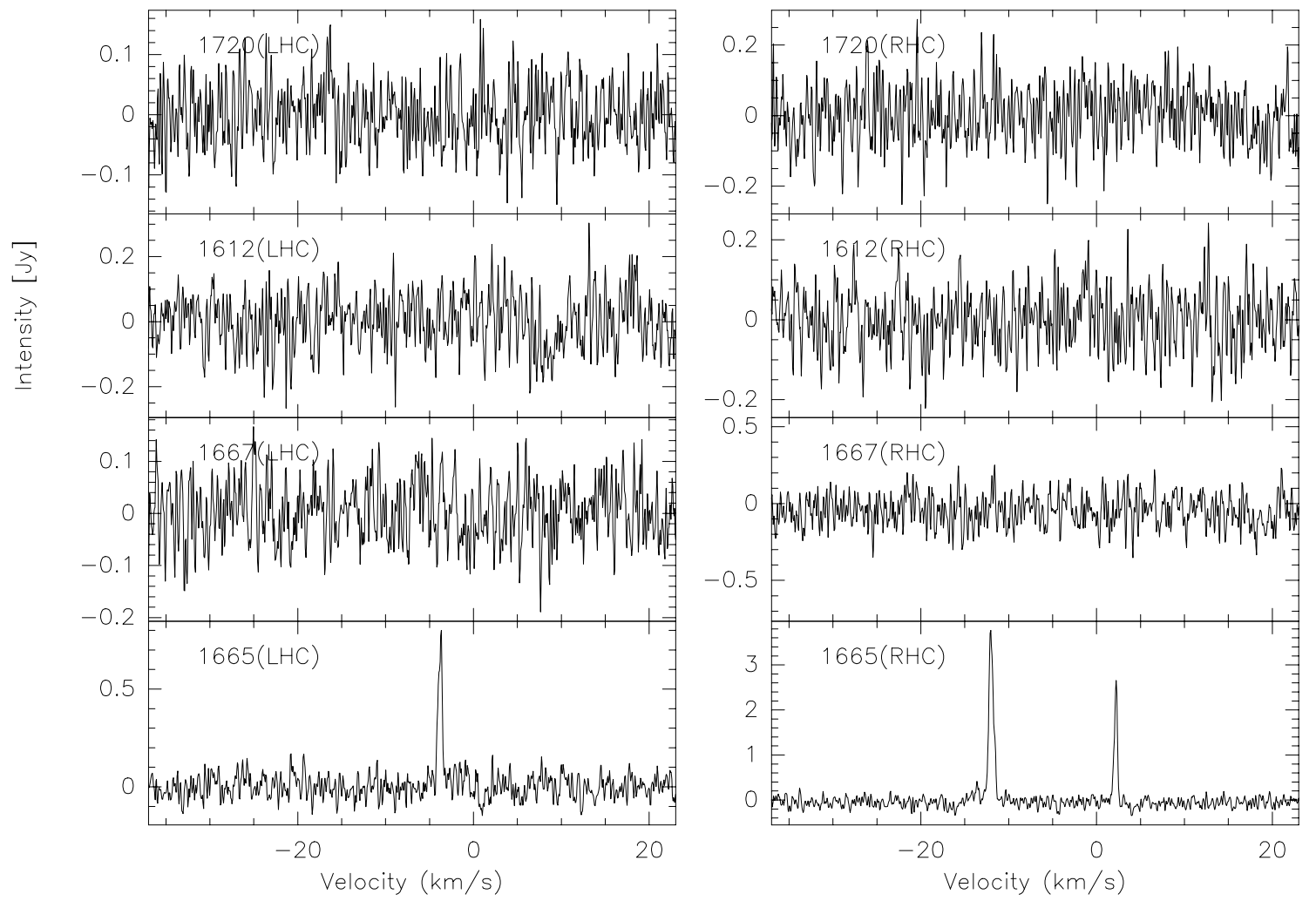

IRAS20188+3928
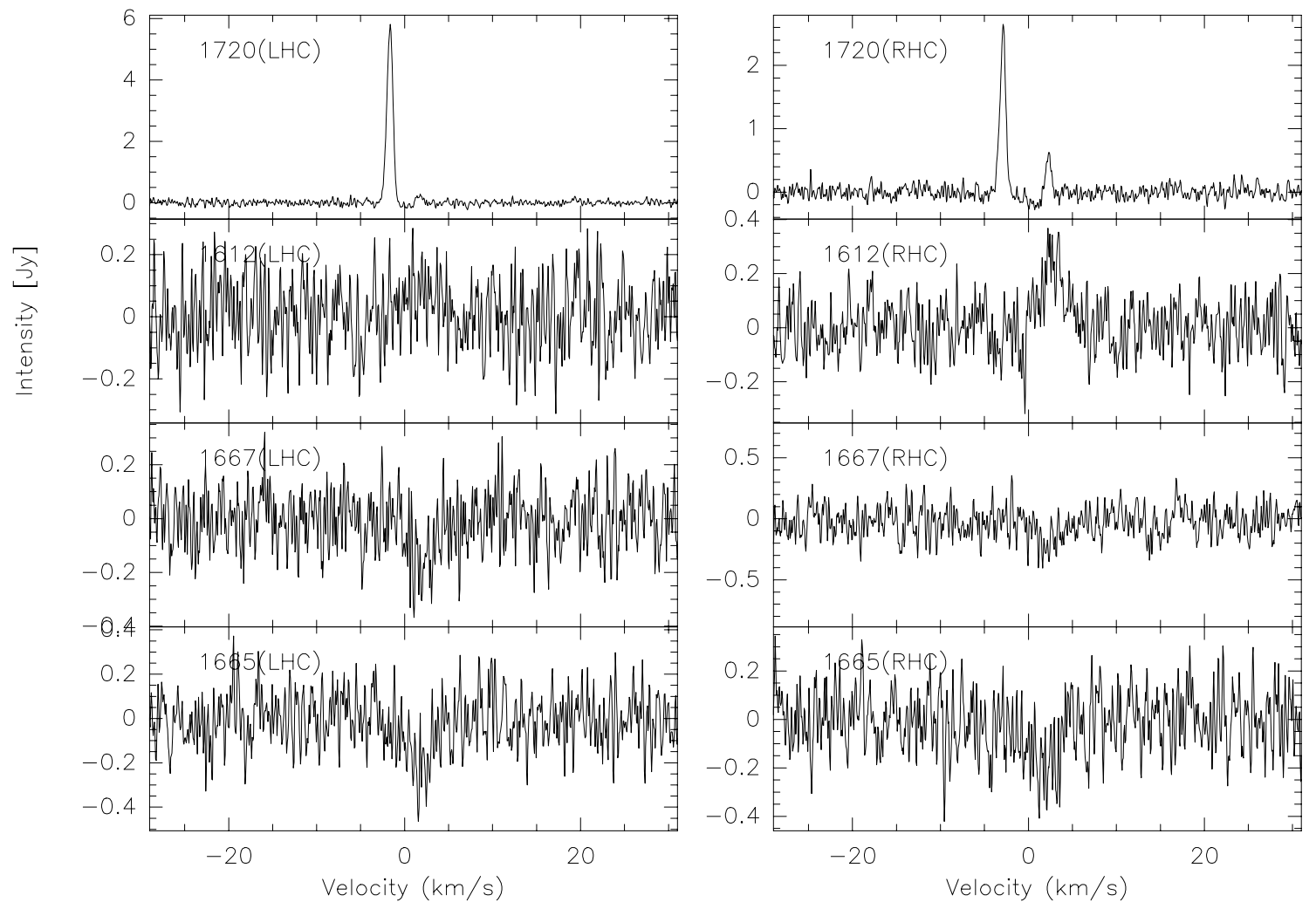

Fig. 19. continued. 
K. A. Edris et al.: OH maser survey towards HMPOs, Online Material p 28

IRAS20227+4154
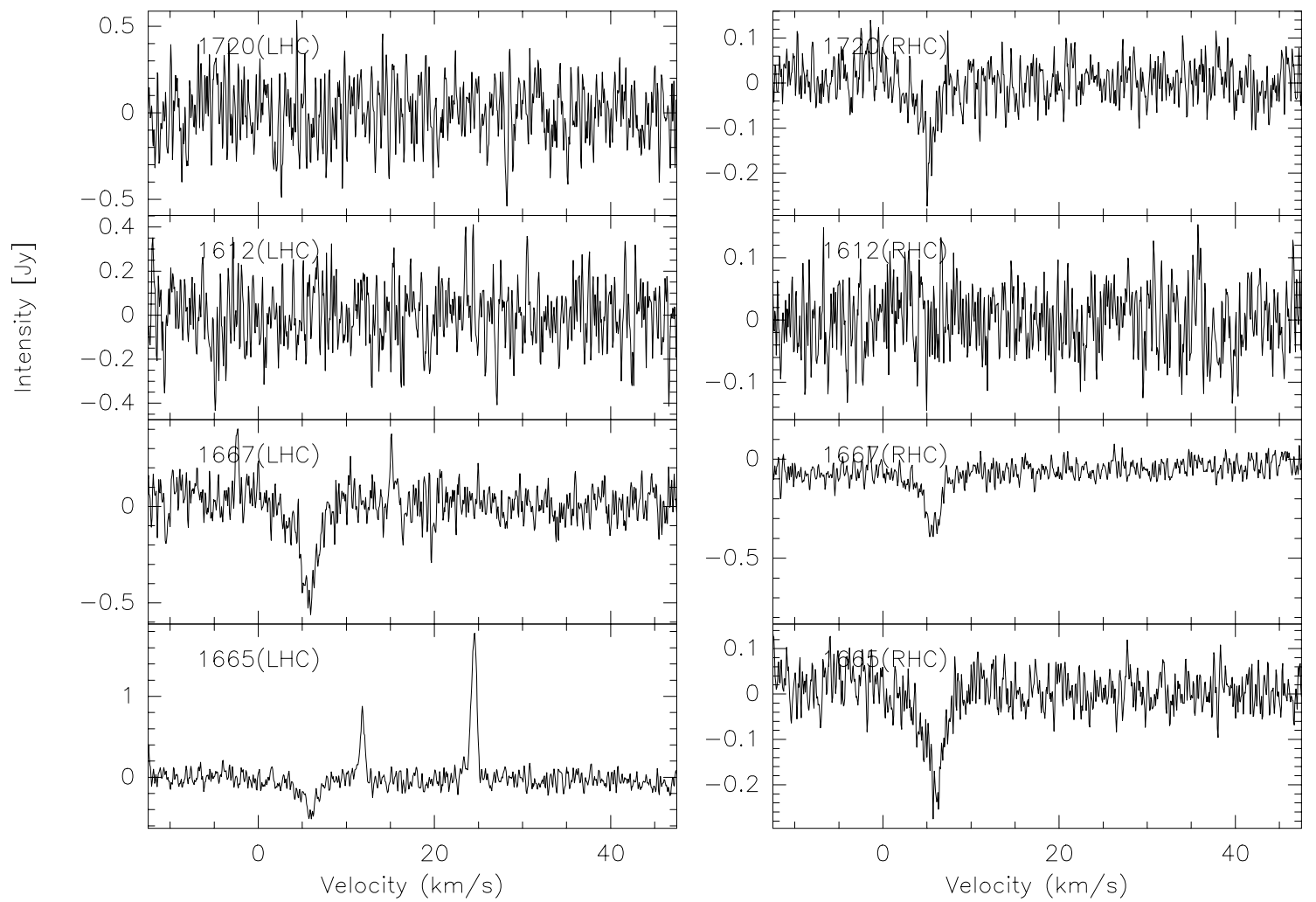

IRAS22198+6336
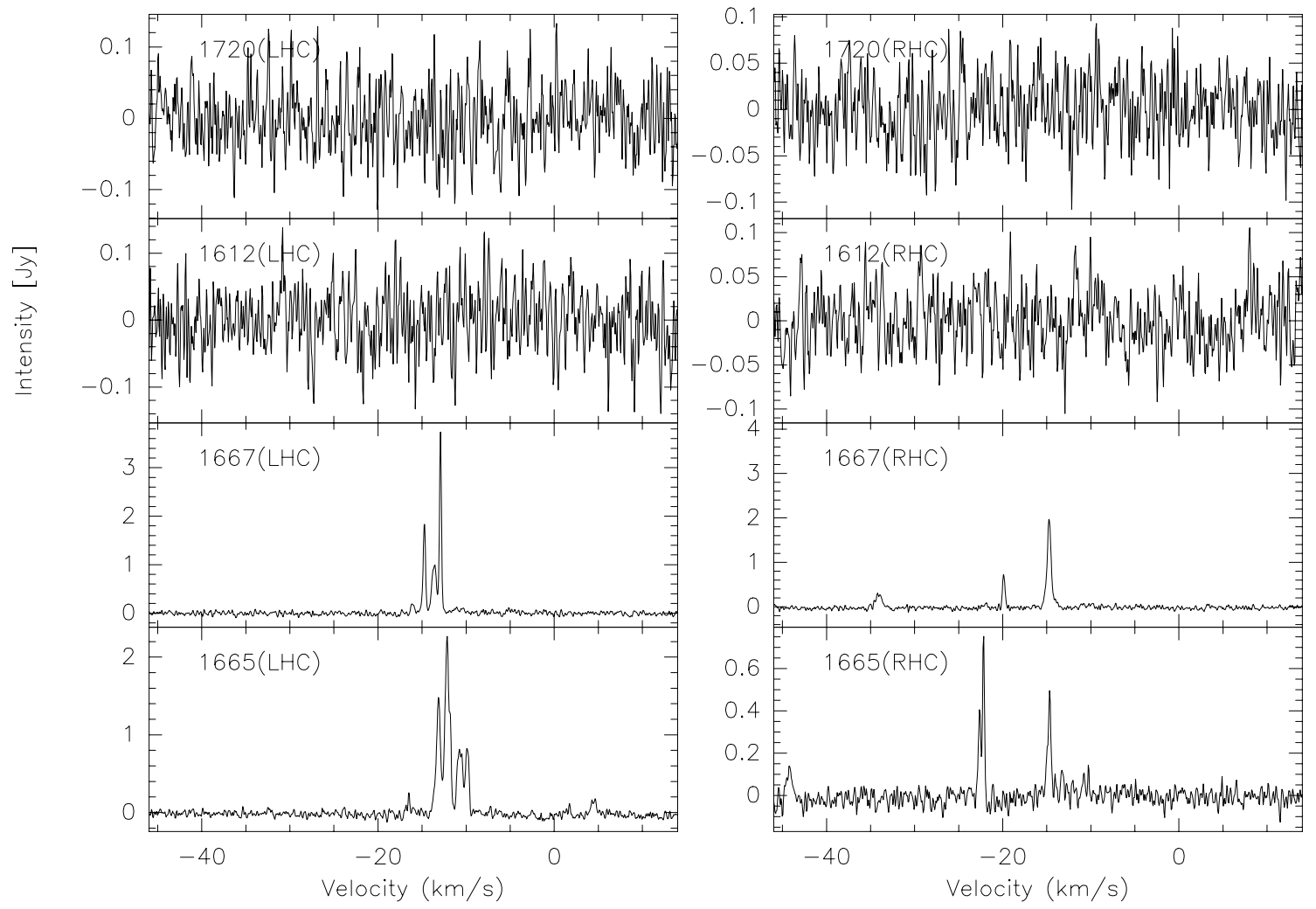

Fig. 19. continued. 
K. A. Edris et al.: OH maser survey towards HMPOs, Online Material p 29

IRAS22272+6358
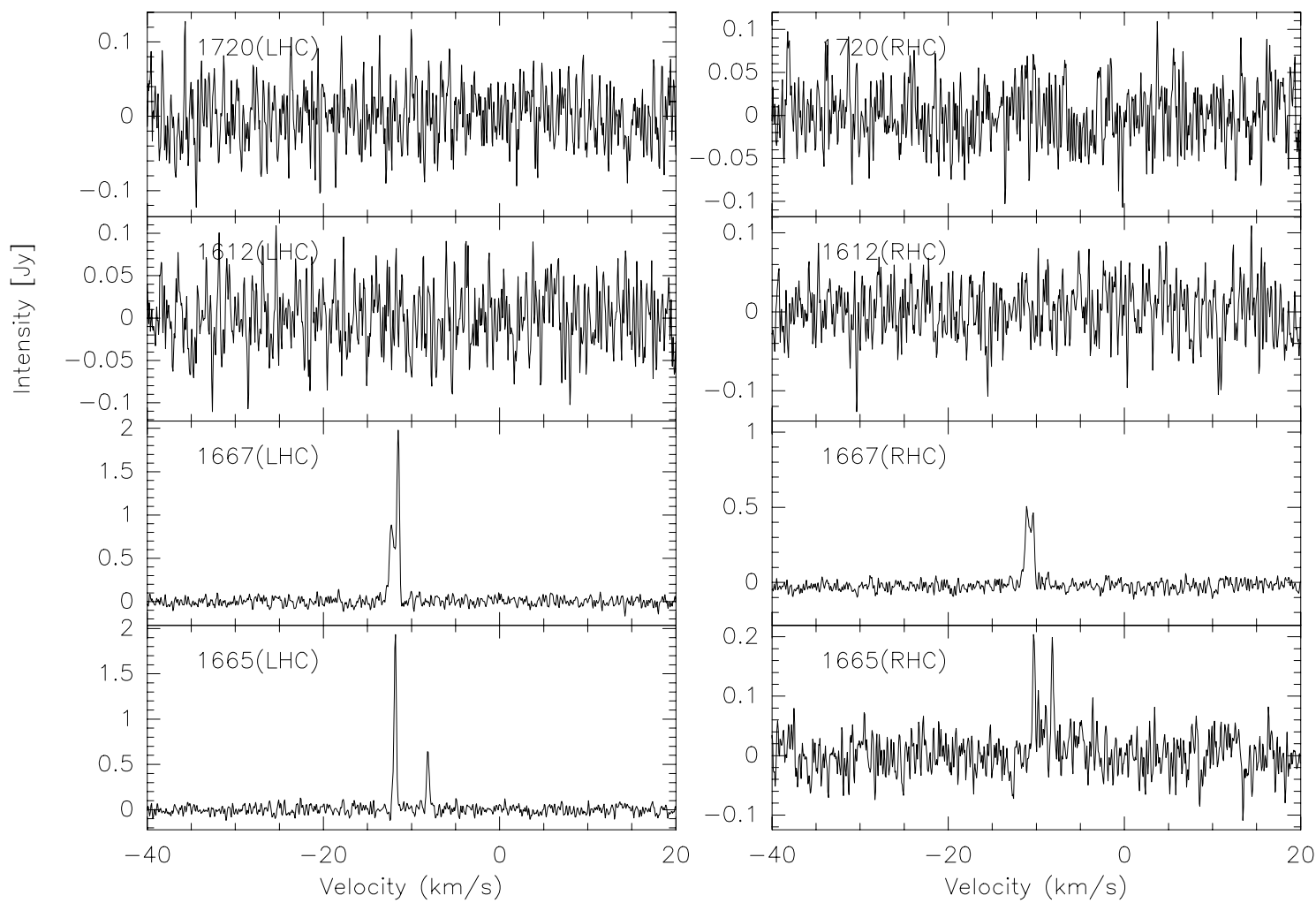

IRAS23139+5939
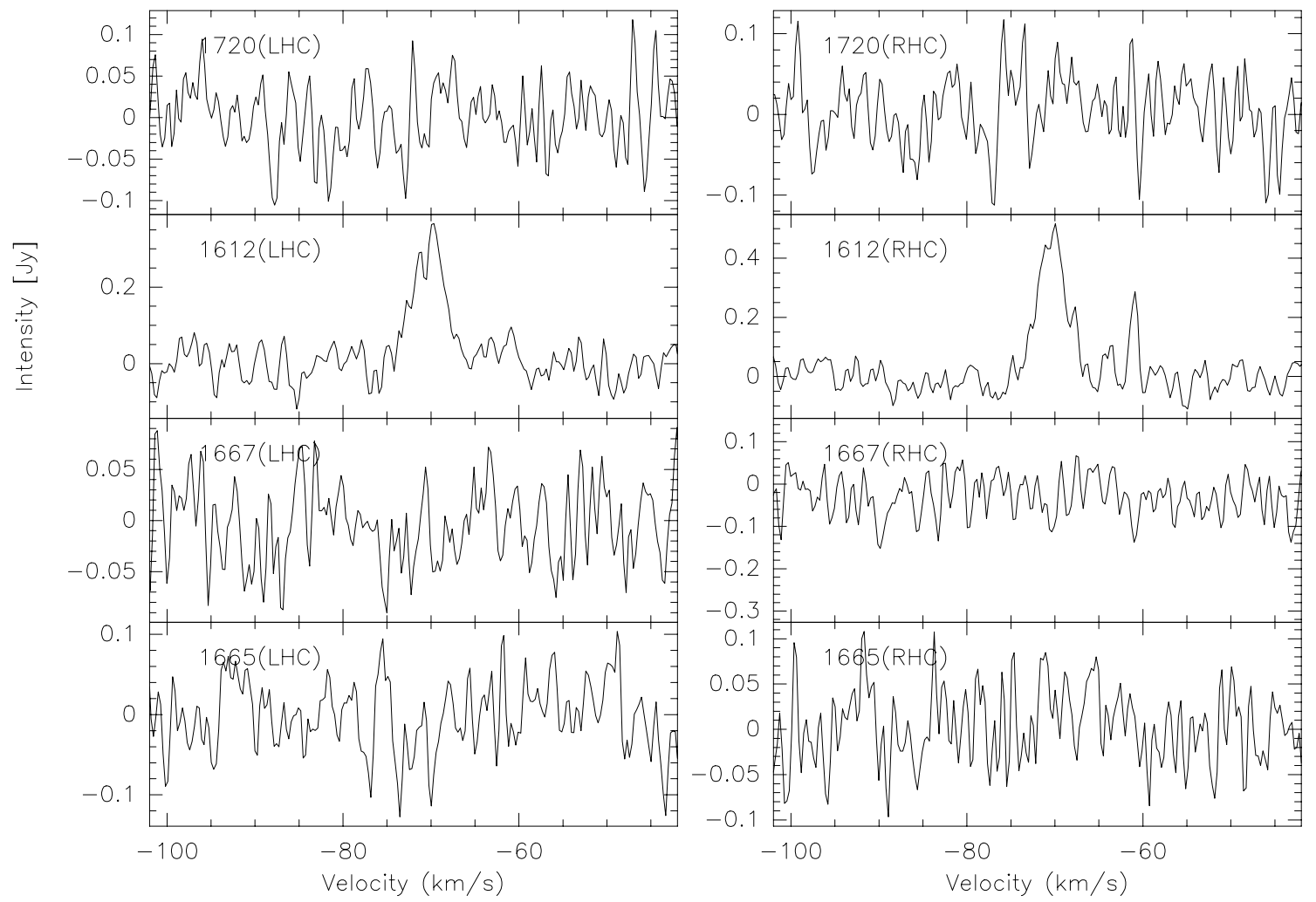

Fig. 19. continued. 
K. A. Edris et al.: OH maser survey towards HMPOs, Online Material p 30

IRAS0 $4579+4703$
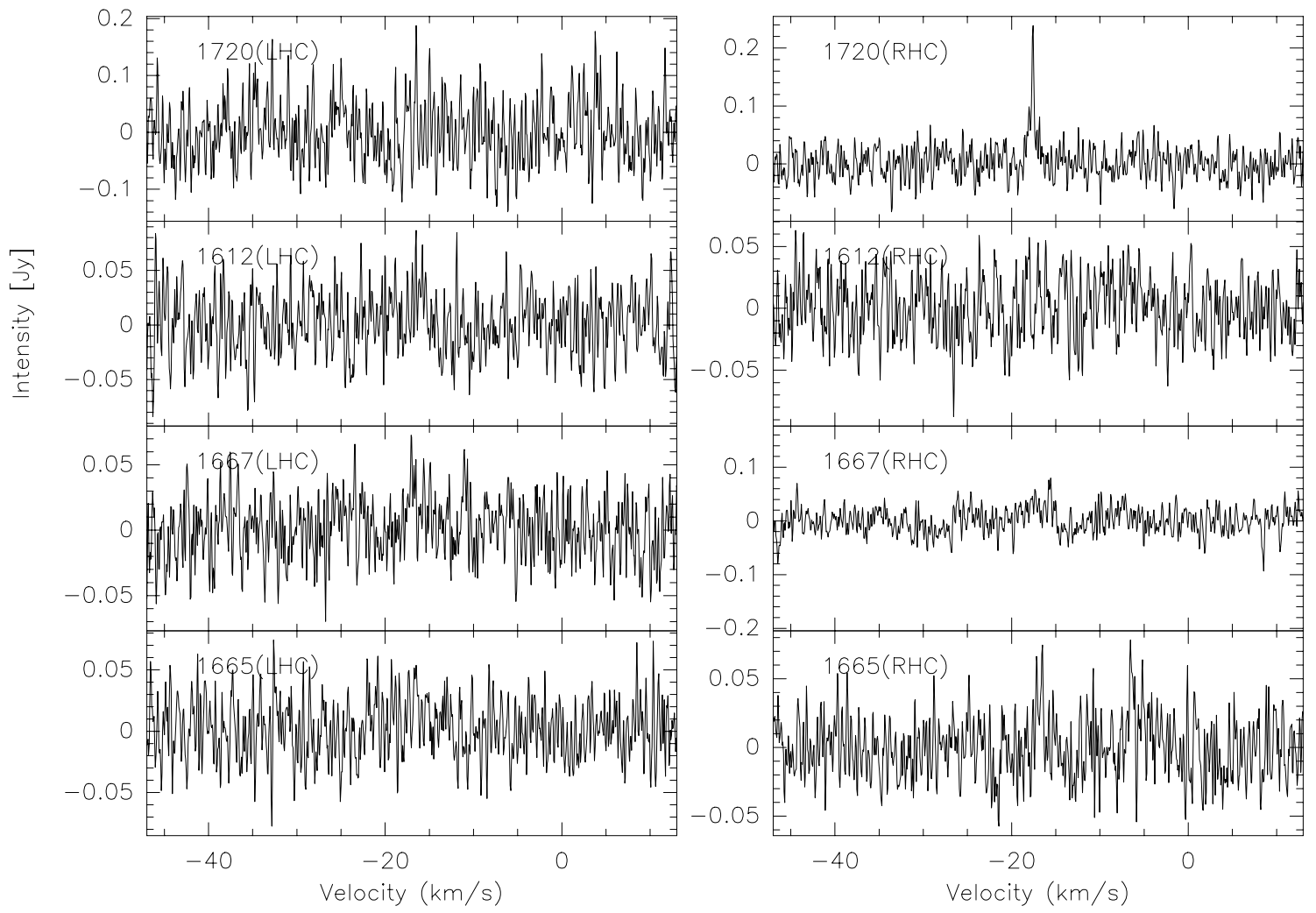

IRAS06382+0939(S)
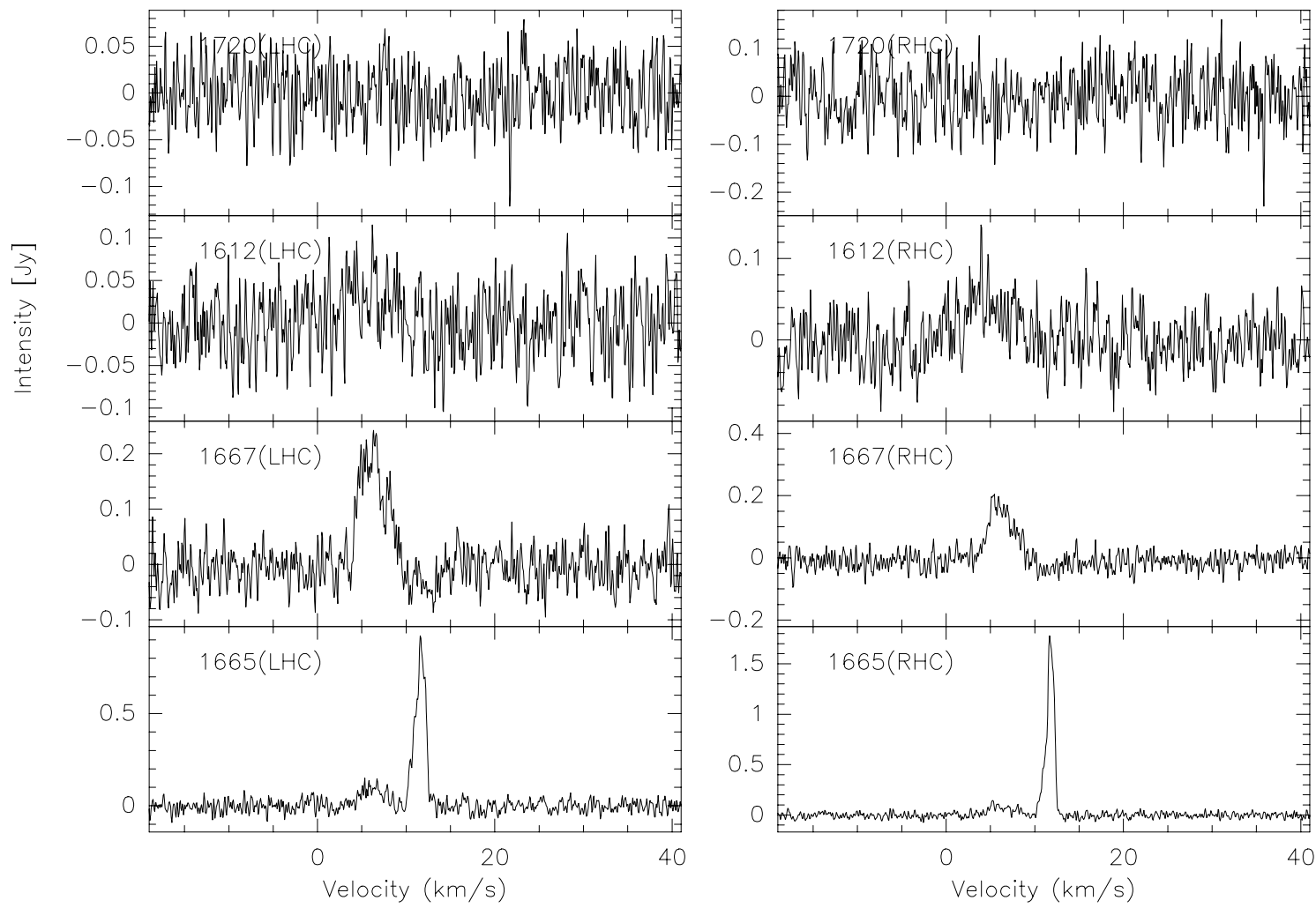

Fig. 20. Same as Fig. 19 but for sources with maser emission offset from the IRAS position by $>2^{\prime}$. 
K. A. Edris et al.: OH maser survey towards HMPOs, Online Material p 31

IRAS18408-0348
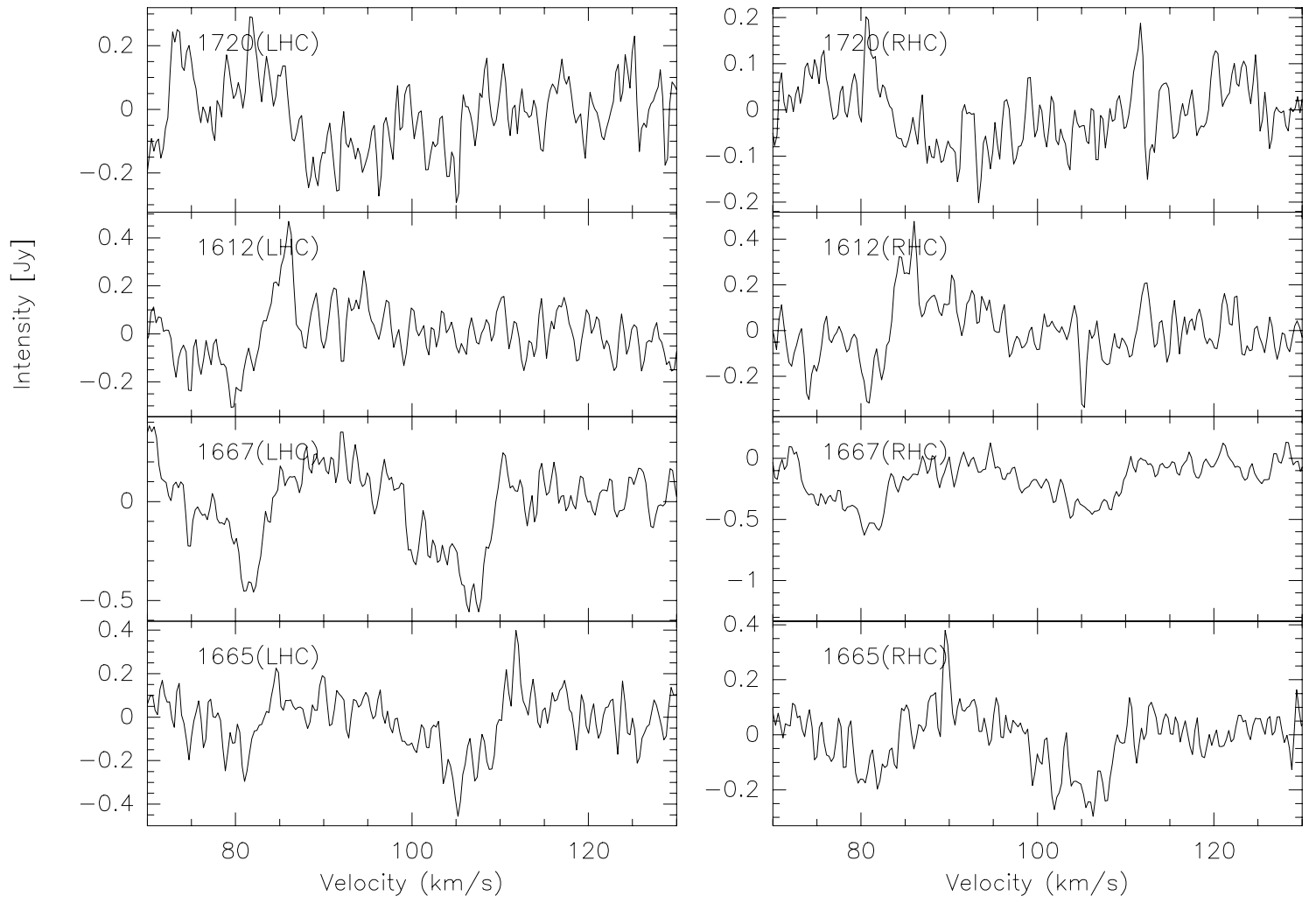

IRAS18511+0146
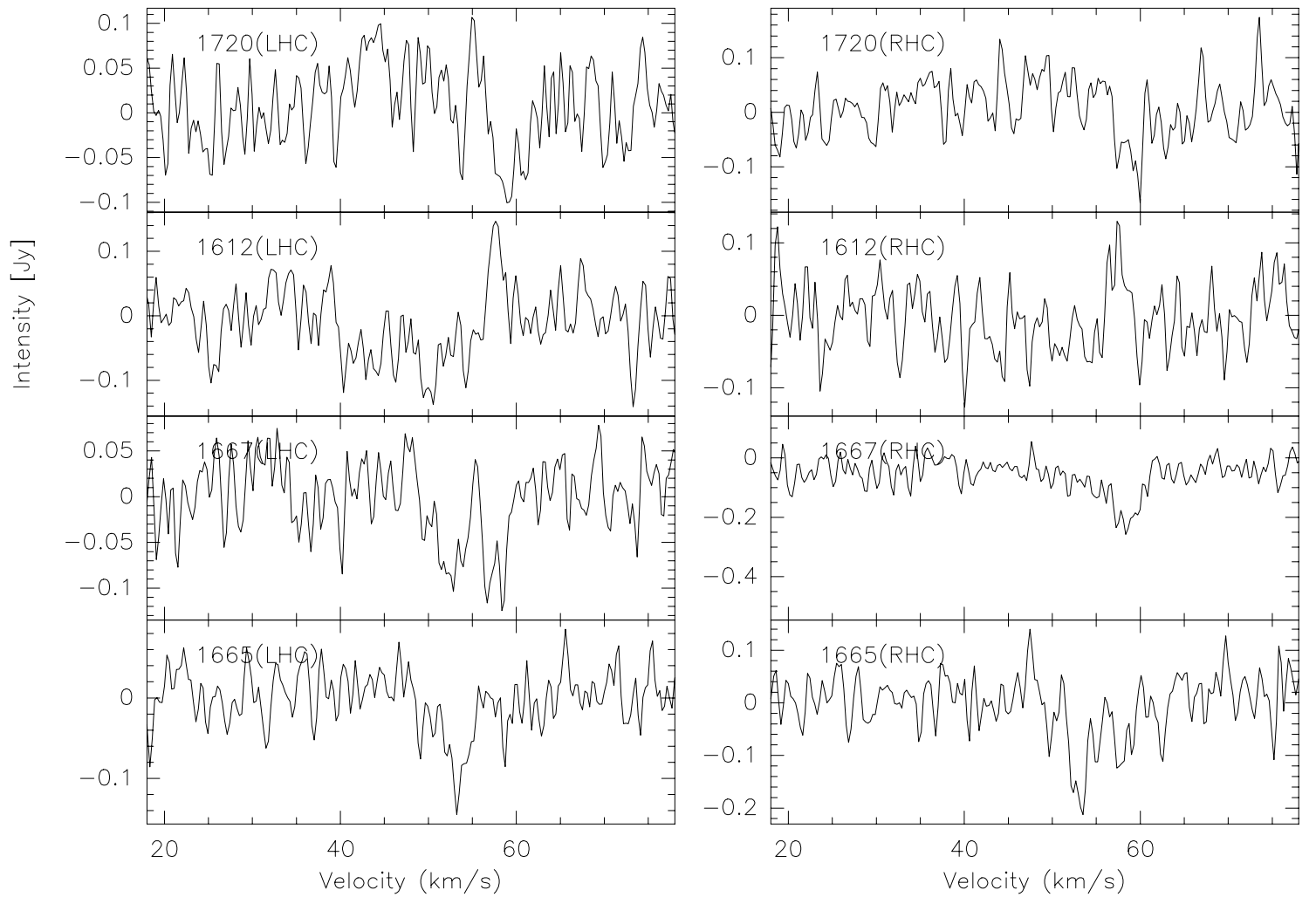

Fig. 20. continued. 
K. A. Edris et al.: OH maser survey towards HMPOs, Online Material p 32

IRAS18540+0220
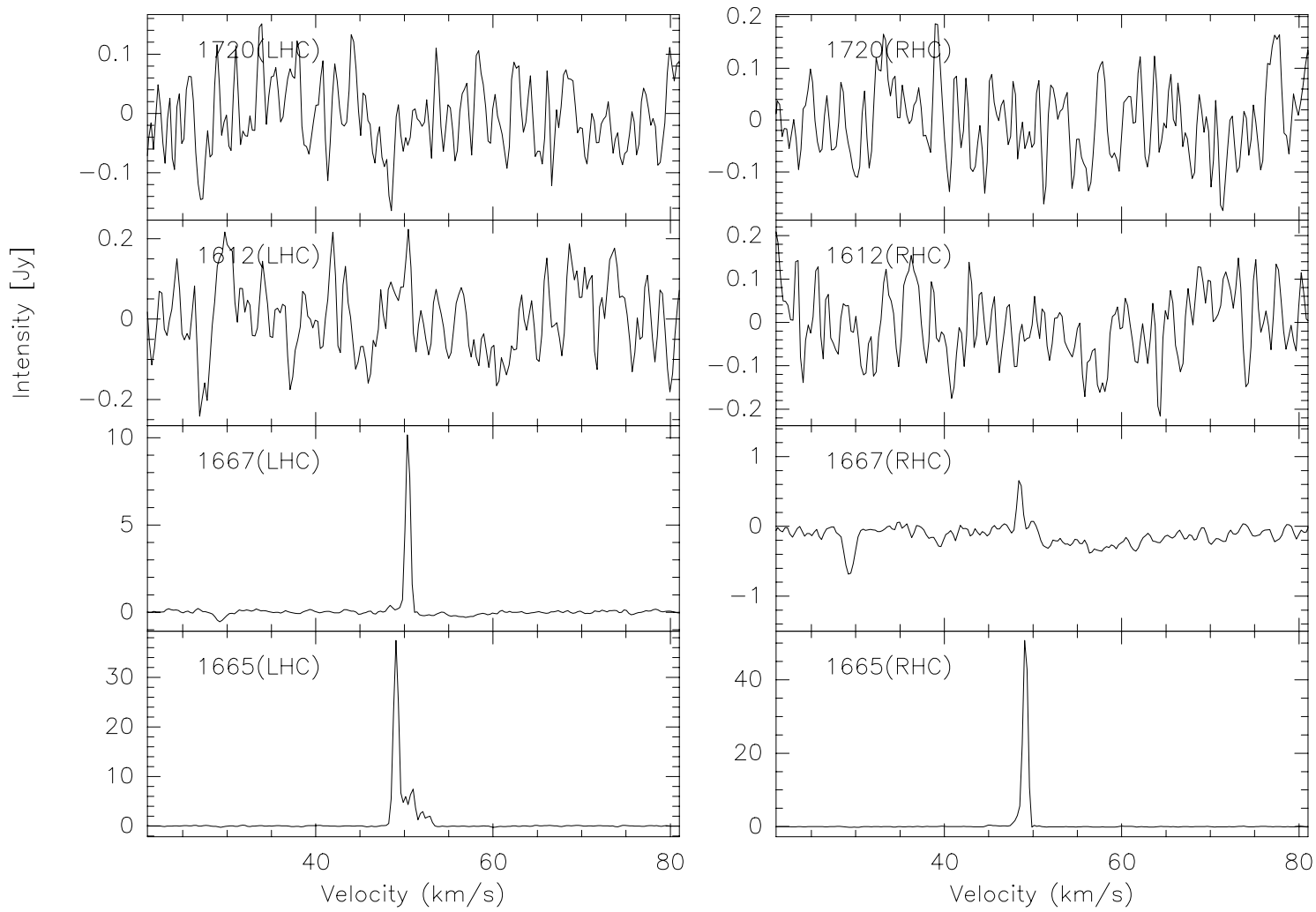

IRAS18586+0106
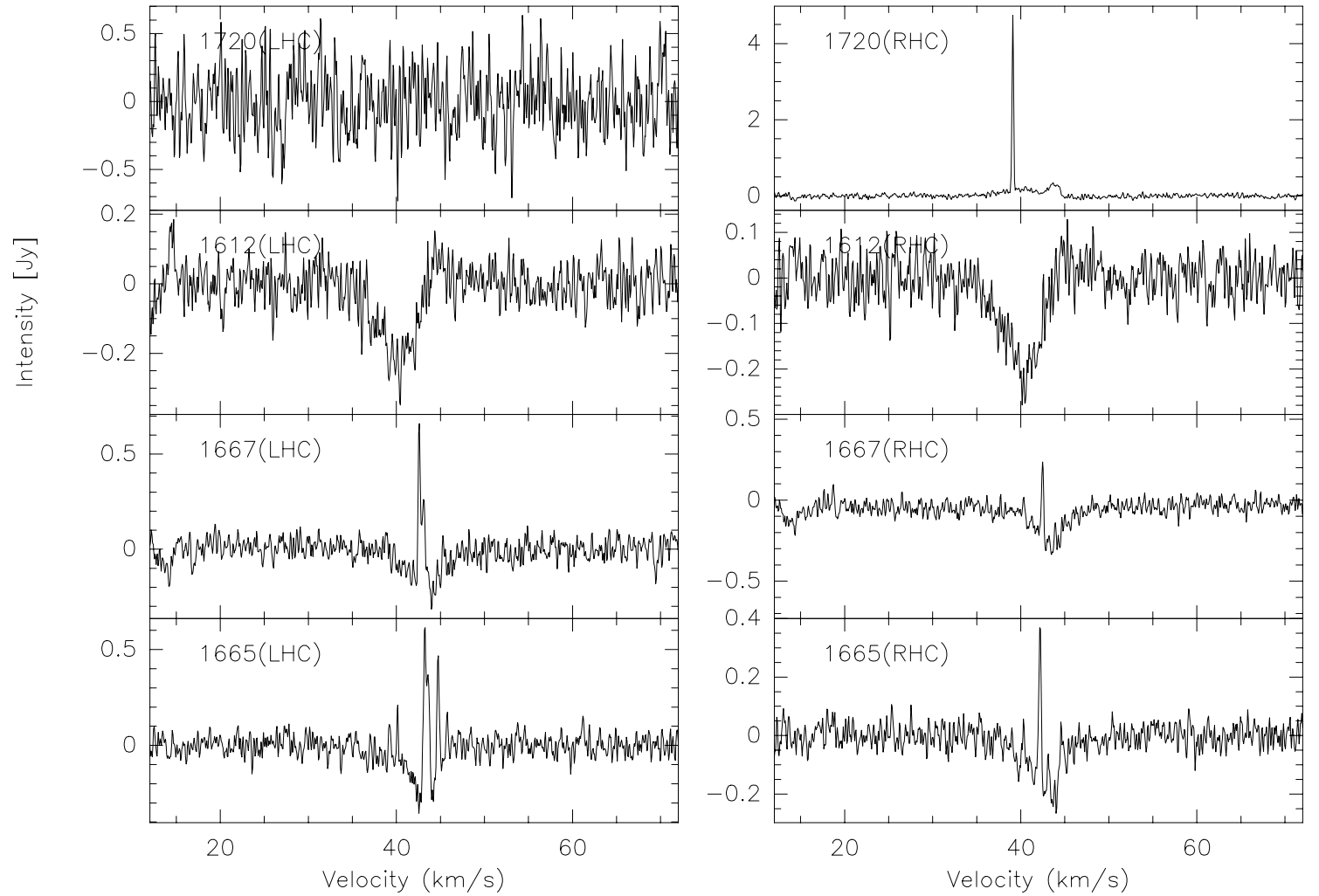

Fig. 20. continued. 
K. A. Edris et al.: OH maser survey towards HMPOs, Online Material p 33

|RAS20099+3640 ||
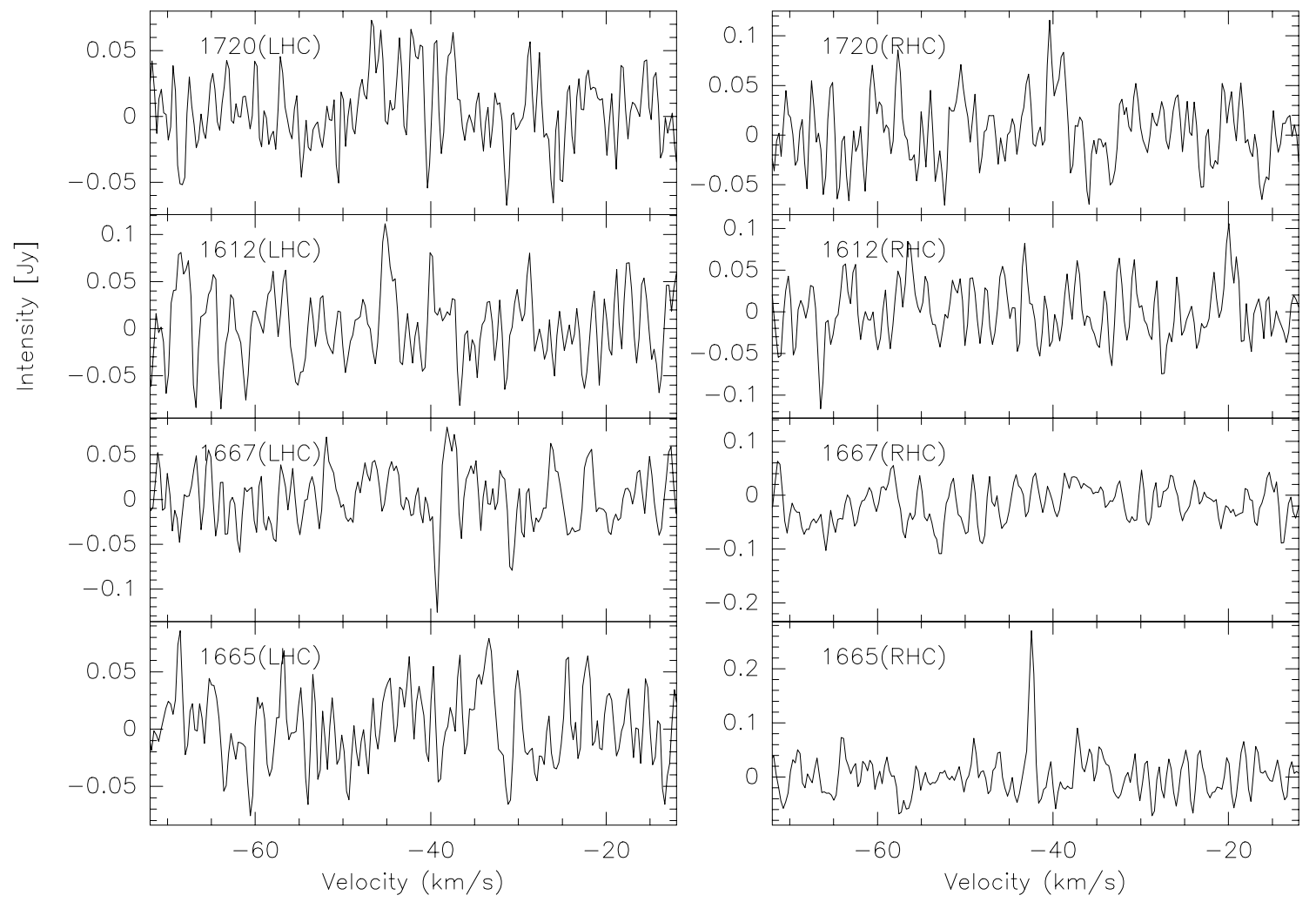

Fig. 20. continued. 
K. A. Edris et al.: OH maser survey towards HMPOs, Online Material p 34

IRAS18258-0737
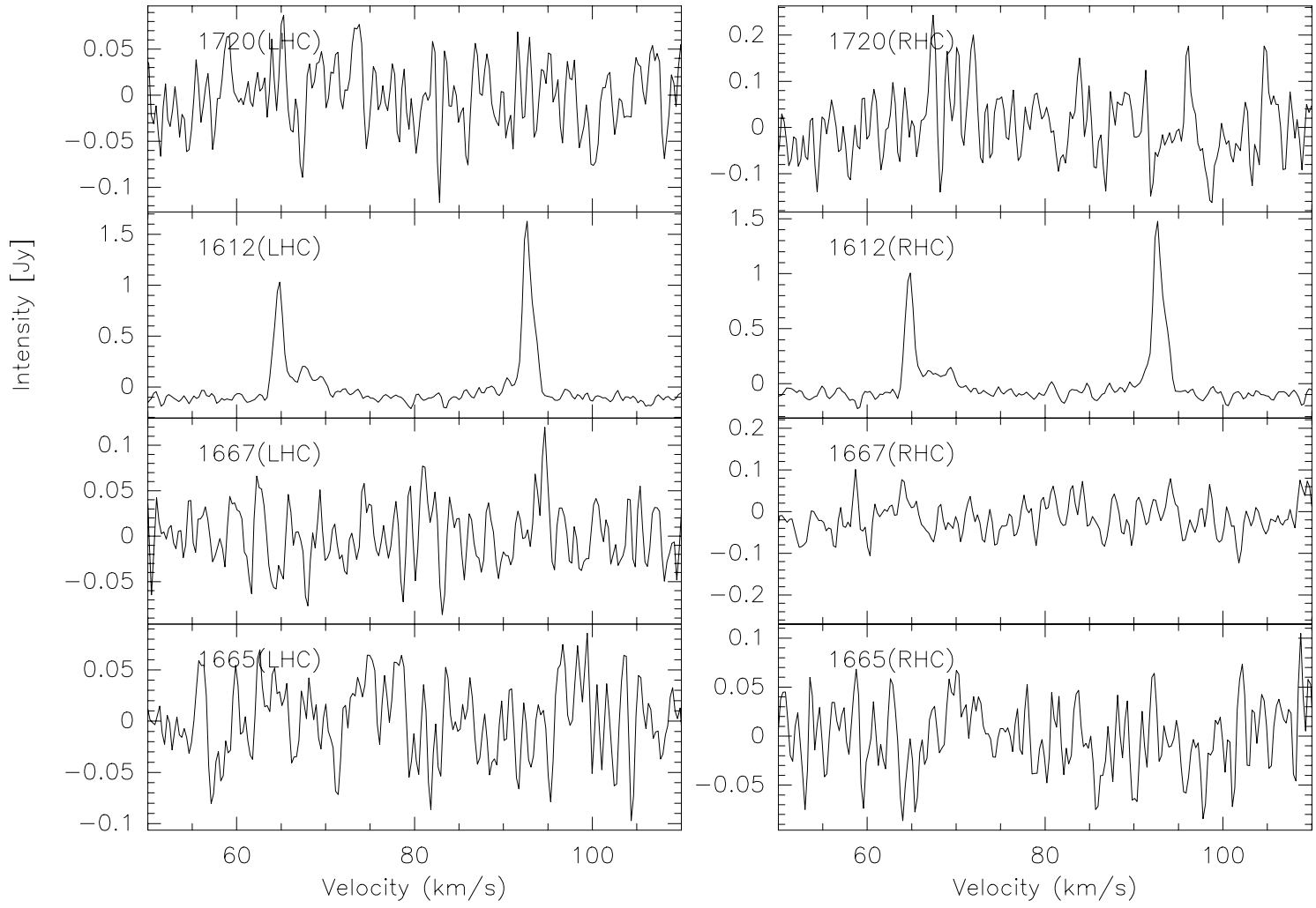

IRAS18348-0616
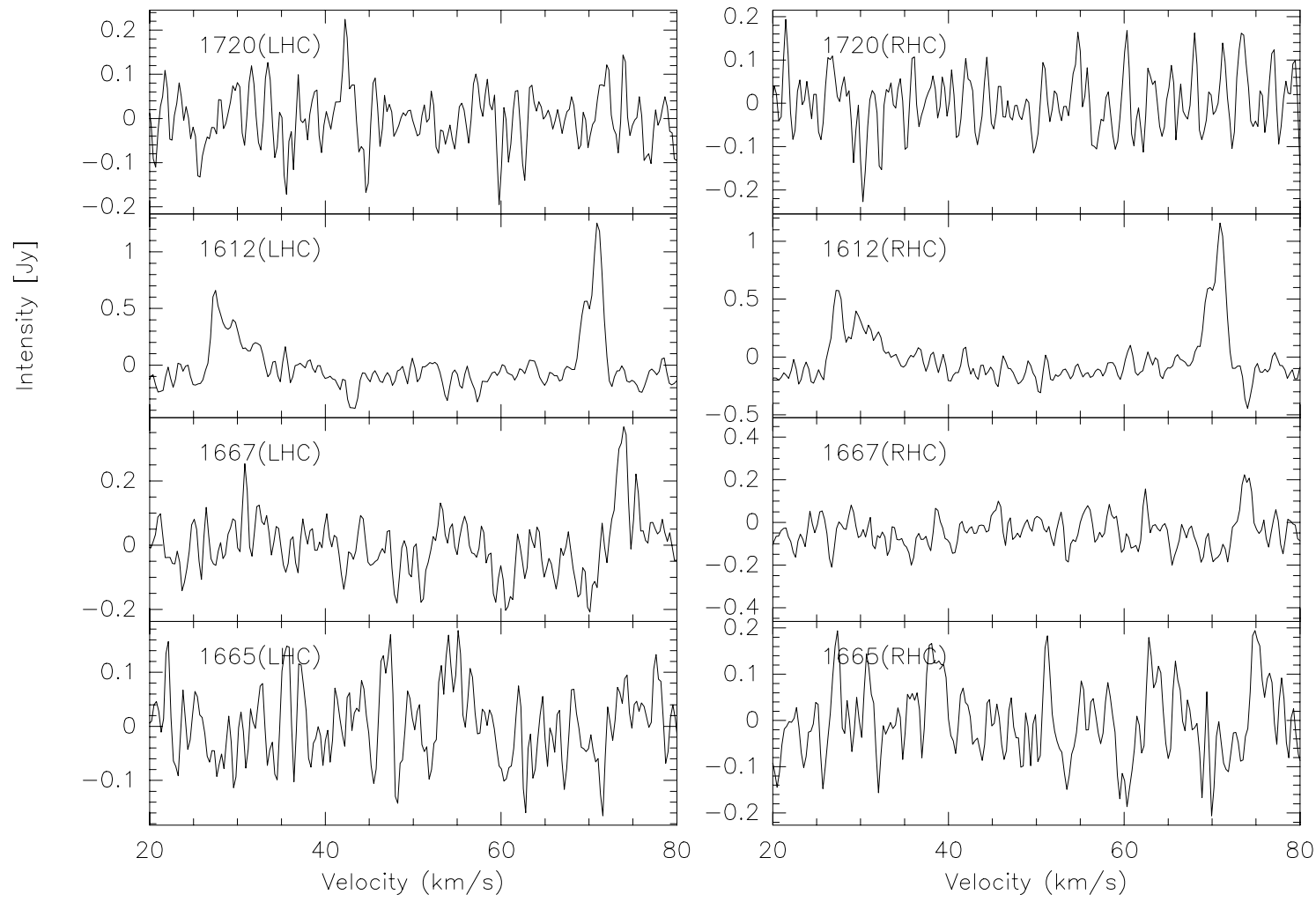

Fig. 20. continued. 
K. A. Edris et al.: OH maser survey towards HMPOs, Online Material p 35

IRAS18424-0329
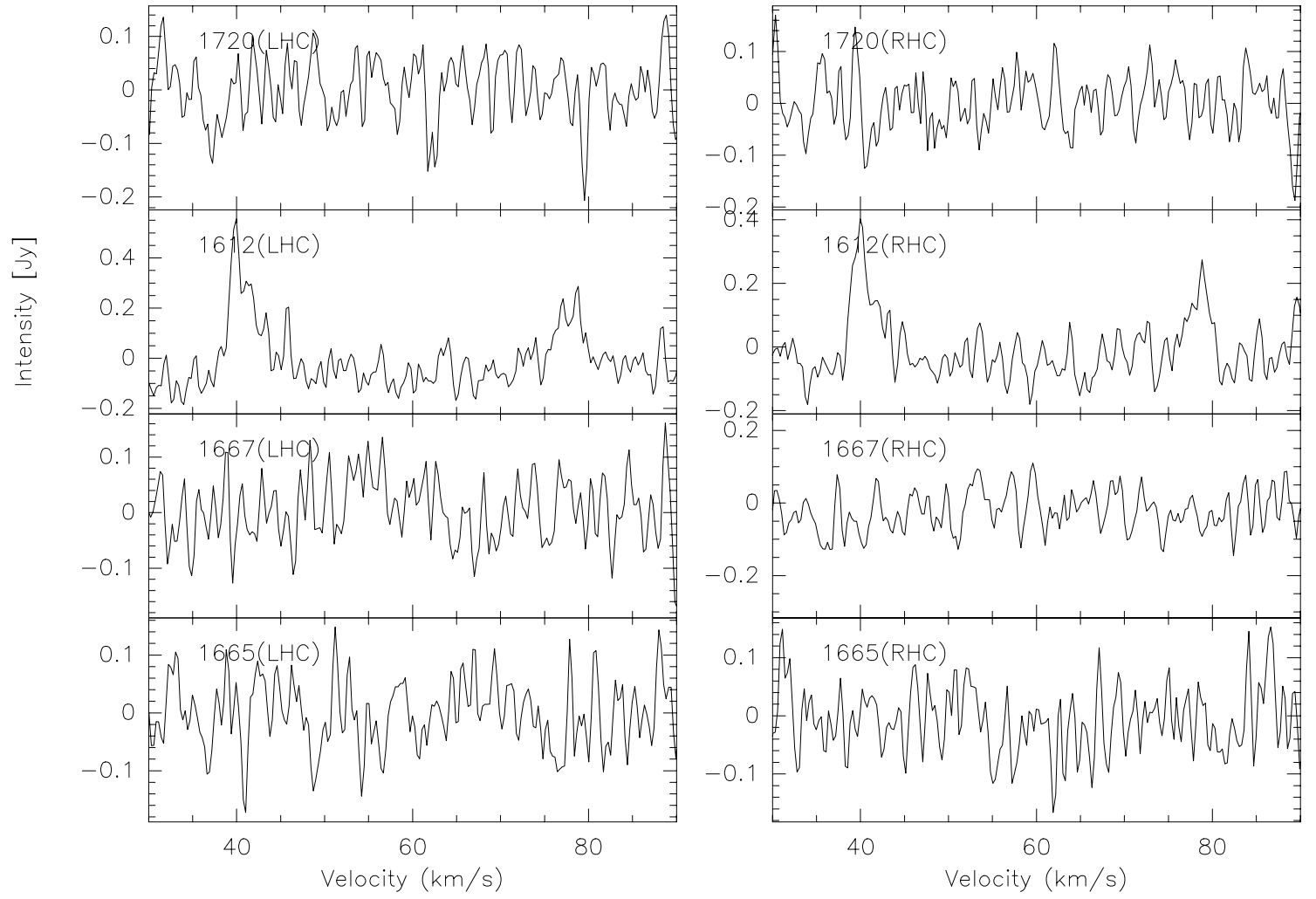

IRAS18565+0349
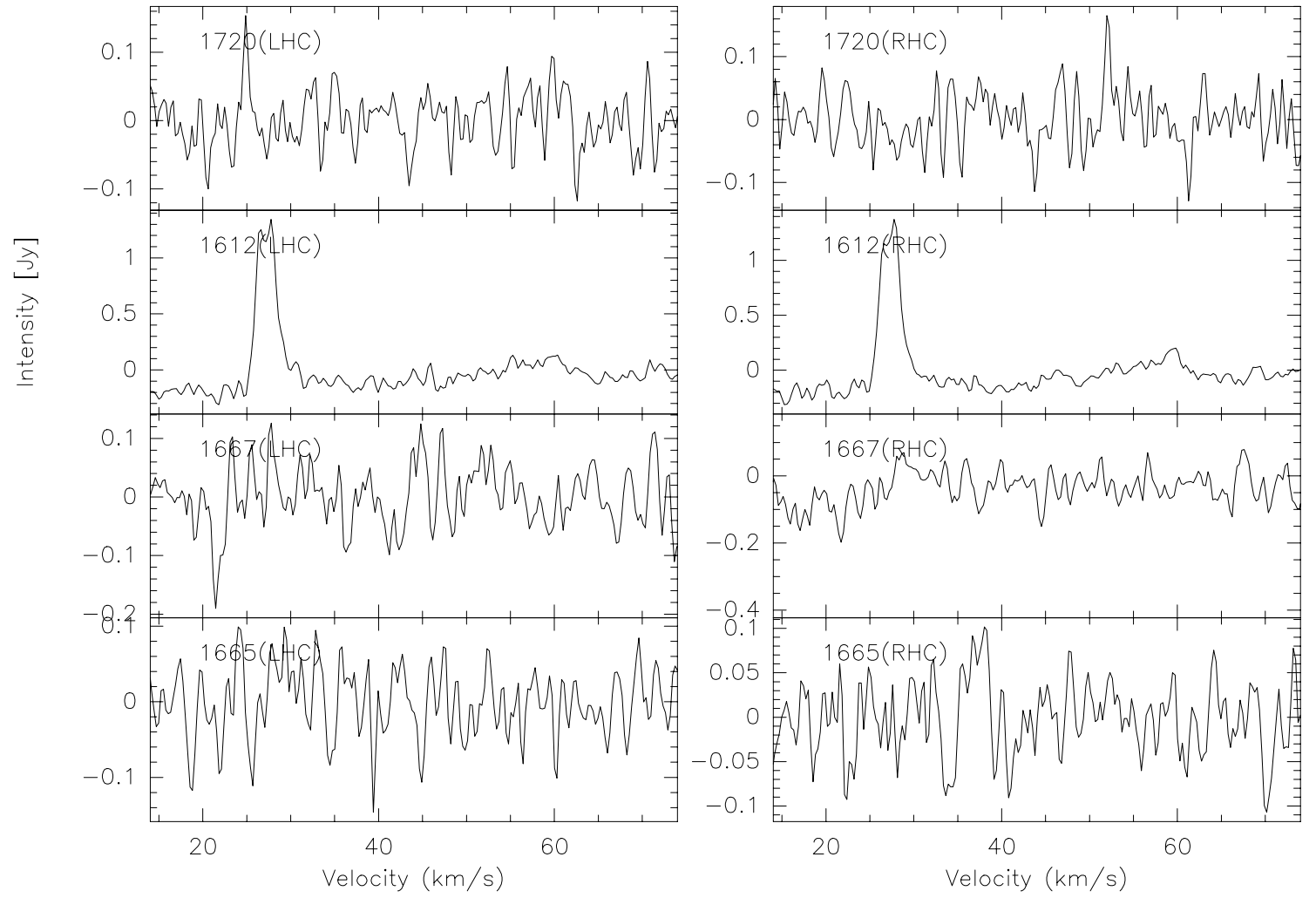

Fig. 20. continued. 


\section{Appendix A: Notes on individual sources where $\mathrm{OH}$ masers were detected}

IRAS 05137+3919. This source also shows water maser emission (P91). This IRAS source is one of the sources studied in detail at high angular resolution by Molinari et al. (2002) at millimeter and centimeter wavelengths in both continuum and spectral lines. There is a core which shows radio continuum emission detected at $3.6 \mathrm{~cm}$ wavelength (VLA1) and mm continuum emission detected at $3.4 \mathrm{~mm}$ wavelength (Fig. 1 of Molinari et al. 2002). At $2.2 \mu \mathrm{m}$ the IRAS source is resolved in to a cluster of objects (Molinari et al. 2002).

IRAS 05274+3345 (AFGL5142). This source is also associated with $\mathrm{H}_{2} \mathrm{O}$ (Verdes-Montenegro et al. 1989; P91) and $\mathrm{CH}_{3} \mathrm{OH}$ (SHK2000) masers. OH maser emission was previously reported towards this source by Braz et al. (1990). The $\mathrm{OH}$ lines detected towards this source, namely 1665,1667 and $1612 \mathrm{MHz}$, have varied since the Braz et al. observations. While the $1665 \mathrm{MHz}$ line has strengthened, the other two lines have weakened.

IRAS 05358+3543 (G173.481+2.445, S231, S233IR). This source has been mapped at high angular resolution, 1.5", with the VLA (see Fig. 27 of Argon et al. 2000). The Nançay-GBT observations detected maser components in the two main lines, 1665 and $1667 \mathrm{MHz}$, in both the left and right circular polarisations, while only four components were reported by Argon et al. in the (LHC) $1665 \mathrm{MHz}$ line. Two components of these four coincide very well with the IRAS source and the others are just $\sim 0.2^{\prime \prime}$ south. Szymczak et al. (2000b) failed to detect maser emission at the $4765 \mathrm{MHz} \mathrm{OH}$ line towards this source. The IRAS source is also associated with $\mathrm{H}_{2} \mathrm{O}$ (S02) and a strong $\mathrm{CH}_{3} \mathrm{OH}$ masers (Galt 2004, and references therein). No radio continuum detection has been reported towards IRAS $05358+3543$ in S02. On the other hand recent sub-millimeter continuum maps revealed a total of four sources (Williams et al. 2004; Minier et al. 2005). One of these sources is coincident with the IRAS source and $\mathrm{OH}$ maser and $~ 40^{\prime \prime}$ north-east another submm source harbours the $\mathrm{CH}_{3} \mathrm{OH}$ maser, exhibits mid-infrared emission and coincides with one of the three 1.2-mm continuum sources detected by Beuther et al. (2002c) (see Fig. 4 of Minier et al. 2005). High angular resolution observations with Plateau de Bure Interferometer by Beuther et al. (2002d) reveal that this region contains at least three molecular outflows.

IRAS 05382+3547. The $\mathrm{OH}$ maser towards this source was discovered by Szymczak \& Kus (2000), but no position for it was measured. GBT observations show that the $1665-\mathrm{MHz}$ OH maser components detected by Szymczak \& Kus centred at velocities -27 and $-22 \mathrm{~km} \mathrm{~s}^{-1}$ are offset from the IRAS position by $\sim 3^{\prime}$. GBT observations found new components centred at velocities -20 and $-24.7 \mathrm{~km} \mathrm{~s}^{-1}$ offset $\sim 30^{\prime \prime}$ northwest of the IRAS source. This source is also associated with a $\mathrm{CH}_{3} \mathrm{OH}$ maser (SHK2000) with velocity centred at $24.1 \mathrm{~km} \mathrm{~s}^{-1}$ close to one of the $\mathrm{OH}$ maser components.

IRAS 06056+2131. Cohen et al. (1988) detected OH maser emission at $1665 \mathrm{MHz}$ only. The Nançay-GBT observations found emission at 1667 and $1720 \mathrm{MHz}$ as well.

IRAS 17527-2439. This source is also coincident with a $\mathrm{H}_{2} \mathrm{O}$ maser (P91).

IRAS 18018-2426 (G6.049-1.447, M8E). This OH maser was first detected by Cohen et al. (1988) and was mapped by Argon et al. (2000). These previous observations detected emission at $1665 \mathrm{MHz}$ only, while Nançay-GBT detected maser emission in the $1667 \mathrm{MHz}$ line as well. The $\mathrm{OH}$ maser emission is variable towards this source (Cohen et al. 1988). The water maser was detected towards this source by Lada et al. (1976) but P91 failed to detect any water maser emission. This source is known to be associated with a compact HII region (Simon et al. 1984). Molinari et al. (2002) detected 6.75 and 4.9 mJy continuum radio emission at 2 and $6 \mathrm{~cm}$ wavelengths respectively. The maser towards this source is not polarized which is unusual (e.g. Cohen 1989). The disappearance of $\mathrm{H}_{2} \mathrm{O}$ masers and the association of $\mathrm{OH}$ maser with compact $\mathrm{HII}$ region suggest that this may be in a late stage of star formation where the UCHII region has expanded and only the $\mathrm{OH}$ maser emission is surviving. No $\mathrm{CH}_{3} \mathrm{OH}$ maser towards this source has been reported.

IRAS 18024-2119. The newly detected $\mathrm{OH}$ main line maser emission towards this source has a relatively wide velocity range, covering $\sim 40 \mathrm{~km} \mathrm{~s}^{-1}$. An $\mathrm{H}_{2} \mathrm{O}$ maser was also detected by P91. A relatively strong $\mathrm{CH}_{3} \mathrm{OH}$ maser of $100 \mathrm{Jy}$ was recently detected by Galt (2004). No molecular outflow was detected towards this source by Zhang et al. (2005).

IRAS 18048-2019. This source shows very weak $\mathrm{OH}$ maser emission in both main lines. Water (P91) and $\mathrm{CH}_{3} \mathrm{OH}$ (Schutte et al. 1993) masers are also associated with this source.

IRAS 18089-1732. This source is common in the S02 sample and the High sub-sample of M96. The $\mathrm{OH}$ maser emission was detected by Cohen et al. (1988) in the $1665 \mathrm{MHz}$ line only. The Argon et al. (2000) VLA map shows several 1665-MHz $\mathrm{OH}$ maser components in three different positions. We detected maser emission at $1667 \mathrm{MHz}$ as well. This source is also associated with $\mathrm{H}_{2} \mathrm{O}$ (P91) and $\mathrm{CH}_{3} \mathrm{OH}$ (SHK2000) masers and is associated with very weak $3.6 \mathrm{~cm}$ continuum emission, $0.9 \mathrm{mJy}$ (S02). Recent high angular resolution submillimeter observations in various spectral lines by Beuther et al. (2005) detect a massive rotating structure perpendicular to an emanating outflow which is likely associated with the central accretion disk.

IRAS 18090-1832. This source also shows a relatively strong, $77 \mathrm{Jy}, \mathrm{CH}_{3} \mathrm{OH}$ maser (SHK2000) and weak OH maser emission in the two main lines.

IRAS 18102-1800. This source is also associated with radio continuum emission, $44 \mathrm{mJy}$, at $3.6 \mathrm{~cm}$ wavelength (S02).

IRAS 18144-1723. This is a relatively strong $\mathrm{OH}$ maser source in the main lines. There is a significant gap, $13 \mathrm{~km} \mathrm{~s}^{-1}$ between the central velocities of strongest components in the 1665 and $1667 \mathrm{MHz}$ lines. The IRAS source is also associated with $\mathrm{CH}_{3} \mathrm{OH}$ (SHK2000) and $\mathrm{H}_{2} \mathrm{O}$ (P91) masers. Radio continuum emission was also detected towards this source at 2 and $6 \mathrm{~cm}$ wavelengths (Molinari et al. 1998).

IRAS 18182-1433. The $\mathrm{H}_{2} \mathrm{O}$ and $\mathrm{CH}_{3} \mathrm{OH}$ masers associated with this source were mapped in high angular resolution, $\sim 1^{\prime \prime}$ by Beuther et al. (2002a). Only one $\mathrm{CH}_{3} \mathrm{OH}$ maser component was detected, while several $\mathrm{H}_{2} \mathrm{O}$ maser components were detected. The $\mathrm{H}_{2} \mathrm{O}$ and $\mathrm{CH}_{3} \mathrm{OH}$ positions are coincident with the $1.2 \mathrm{~mm}$ continuum emission detected by Beuther et al. (2002c). Extremely weak radio continuum emission has been detected by Foster \& Caswell (2000) $(0.3 \mathrm{mJy}$ at $3.5 \mathrm{~cm})$ and S02 $(<1 \mathrm{mJy}$ at $3.6 \mathrm{~cm})$. An outflow was detected towards this source by Beuther et al. (2002b).

IRAS 18236-1205. This IRAS source is also associated with $\mathrm{CH}_{3} \mathrm{OH}$ (SHK2000) and $\mathrm{H}_{2} \mathrm{O}$ (P91) masers.

IRAS 18264-1152. This source shows maser emission at

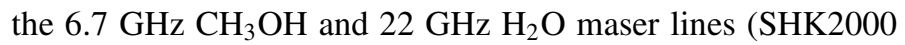
and $\mathrm{P} 91$ respectively). No radio continuum emission, $<1 \mathrm{mJy}$, is detected at $3.6 \mathrm{~cm}$ wavelength (S02). An outflow was detected towards this source by Beuther et al. (2002b). 
IRAS 18278-1009. This source is also associated with a $\mathrm{CH}_{3} \mathrm{OH}$ maser (SHK2000). No radio continuum emission was detected at 2 and $6 \mathrm{~cm}$ wavelengths by Molinari et al. (1998).

IRAS 18290-0924. This source is also associated with $\mathrm{H}_{2} \mathrm{O}$ and $\mathrm{CH}_{3} \mathrm{OH}$ masers (S02). The mm observations (Beuther et al. 2002c) towards this source show two peaks separated by $12^{\prime \prime}$ with the masers coincident with one of them. This source is also associated with radio continuum emission at $3.6 \mathrm{~cm}$ detected by $\mathrm{S} 02$.

IRAS 18310-0825. This source is the only source in our sample associated only with the $1667-\mathrm{MHz}$ of $\mathrm{OH}$ maser lines. It is also associated with a $\mathrm{CH}_{3} \mathrm{OH}$ maser detected by SHK2000 and mapped by Beuther et al. (2002a). The methanol maser is offset from the IRAS source and coincident with one of two $\mathrm{mm}$ and $\mathrm{cm}$ peaks (see Fig. 1 of Beuther et al. 2002a).

IRAS 18316-0602. This source is also associated with very strong $\mathrm{H}_{2} \mathrm{O}$ (725.83 Jy, P91), and $\mathrm{CH}_{3} \mathrm{OH}$ (178 Jy, SHK2000) masers. This source is known to be associated with an UCHII region (Jenness et al. 1995) and molecular outflow (Wu et al. 2004). The $\mathrm{OH}$ maser emission is detected in the main lines while the satellite lines show conjugate behaviour with thermal emission at $1612 \mathrm{MHz}$ and absorption at $1720 \mathrm{MHz}$.

IRAS 18345-0641. Towards this star-forming region only the $1612 \mathrm{MHz} \mathrm{OH}$ maser line was detected. The IRAS source is associated with strongly, variable $\mathrm{CH}_{3} \mathrm{OH}$ masers (SHK2000) which coincide with a mm continuum peak (Beuther et al. 2002a). Also free-free emission at $3.6 \mathrm{~cm}$ (S02) and an outflow have been detected towards this IRAS source (Beuther et al. 2002b).

IRAS 18360-0537. In addition to the $\mathrm{OH}$ main line masers detected here, this source is also associated with relative strong $\mathrm{H}_{2} \mathrm{O}$ maser emission, $92.73 \mathrm{Jy}$ (P91).

IRAS 18385-0512. This source is also associated with a relatively strong $\mathrm{H}_{2} \mathrm{O}$ maser emission, $200 \mathrm{Jy}$ (S02). Several $\mathrm{H}_{2} \mathrm{O}$ maser components coincide with a mm continuum peak (Beuther et al. 2002a). Radio continuum emission of 29 mJy was measured by $\mathrm{S} 02$ at $3.6 \mathrm{~cm}$.

IRAS 18440-0148. This source is also associated with a $\mathrm{CH}_{3} \mathrm{OH}$ maser (SHK2000; Walsh et al. 1998). The $\mathrm{CH}_{3} \mathrm{OH}$ maser components coincident with a $1.2 \mathrm{~mm}, 3.6 \mathrm{~cm}$ peak and mid-infrared source (Beuther et al. 2002a; S02). A tentative detection of $\mathrm{H}_{2} \mathrm{O}$ maser emission is reported by $\mathrm{S} 02$.

IRAS 18454-0158. This is one of the sources which has an $\mathrm{OH}$ maser but does not have any other known masers. S02 observations did not detect $\mathrm{H}_{2} \mathrm{O}$ or $\mathrm{CH}_{3} \mathrm{OH}$ maser emission. This source is associated with $1.2 \mathrm{~mm}$ continuum emission as well as radio continuum emission (Beuther et al. 2002c and S02 respectively).

IRAS 18463+0052. Only $1612 \mathrm{MHz}$ line OH masers are detected towards this source with a line profile with peaks at 67 and $92 \mathrm{~km} \mathrm{~s}^{-1}$, suggesting this source is an $\mathrm{OH} / \mathrm{IR}$ star rather than a star-forming region. There are no $\mathrm{H}_{2} \mathrm{O}$ or $\mathrm{CH}_{3} \mathrm{OH}$ masers associated with the IRAS source.

IRAS 18488+0000. This is one of the common sources in the S02 sample and the High sub-sample of M96. This source is associated with a variable $\mathrm{CH}_{3} \mathrm{OH}$ maser (SHK2000, and references therein). Although not detected by $\mathrm{P} 91, \mathrm{H}_{2} \mathrm{O}$ maser emission has been detected by S02. The water maser is coincident within few arcsecond with a mid-infrared source but offset from a millimetre continuum source (Beuther et al. 2002a). Relatively strong radio continuum emission, $194 \mathrm{mJy}$, has been detected towards this source (S02). Our Nançay-GBT observations detect $\mathrm{OH}$ maser emission in the two main lines which is coincident (within $\sim 30^{\prime \prime}$ ) with the $1.2 \mathrm{~mm}$ emission source rather than other tracers.

IRAS 18507+0121. This source is roughly $11^{\prime}$ north from $\mathrm{G} 34.257+0.154$ (or $\mathrm{G} 34.3+0.2$ ) which shows strong $\mathrm{OH}$ maser emission in the main lines as well as $1720 \mathrm{MHz}$ satellite line (Argon et al. 2000). G34.257+0.154 also has $\mathrm{H}_{2} \mathrm{O}$ masers and is classified as an HII region (Benson \& Johnston 1984; Genzel \& Downes 1977). The Nançay-GBT observations detect new (relatively weaker) $\mathrm{OH}$ maser emission associated with the IRAS source in the main lines. The IRAS source is also associated with $\mathrm{H}_{2} \mathrm{O}$ (P91) and $\mathrm{CH}_{3} \mathrm{OH}$ (SHK2000) masers. IRAS $18507+0121$ region was studied in detail by Shepherd et al. (2004) at several millimeter and near-infrared (NIR) wavelengths. Shepherd et al. detected two compact molecular cores separated by $\sim 40^{\prime \prime}$ north-south. The northern molecular core contains a newly discovered, deeply embedded, B2 protostar surrounded by several hundred solar masses of warm gas and dust, G34.4+0.23 MM. Based on the presence of warm dust emission and the lack of detection at NIR wavelengths, Shepherd et al. suggest that G34.4+0.23 MM may represent the relatively rare discovery of a massive protostar (analogous to a low-mass "Class 0" protostar). The southern molecular core is associated with an NIR cluster of young stars and an UCHII region, G34.4+0.23 (detected by Miralles et al. 1994), with a central B0.5 star. Shepherd et al. to suggest an upper limit on the age of the IRAS $18507+0121$ star-forming region of $3 \mathrm{Myr}$. This IRAS source is not associated with molecular outflow (Zhang et al. 2005).

IRAS 18527+0301. This source was searched for $\mathrm{OH}$ masers by Szymczak \& Kus (2000) to a rms noise level of 0.2 Jy but none were detected. The present observations, with better sensitivity ( $0.02 \mathrm{Jy})$, detected weak emission in the two main lines. Methanol maser emission was detected by SHK2000 in the same $\mathrm{OH}$ velocity range. No radio continuum emission at $6 \mathrm{~cm}$ was detected by Molinari et al. (1998).

IRAS 18553+0414. This source is also associated with $\mathrm{H}_{2} \mathrm{O}$ maser emission ( $\mathrm{S} 02$ ) which is coincidence with a millimeter continuum source (Beuther et al. 2002c).

IRAS 18566+0408. This common source in the S02 sample and the High sub-sample of M96 is also associated with $\mathrm{CH}_{3} \mathrm{OH}$ maser emission ( $\mathrm{S} 02$; $\mathrm{SHK} 2000$ ). The $\mathrm{H}_{2} \mathrm{O}$ maser emission was newly detected by $\mathrm{S} 02$, while not detected by P91. The $\mathrm{H}_{2} \mathrm{O}$ masers are in better agreement with a millimeter continuum (Beuther et al. 2002a). The newly detected OH maser emission also seemingly coincides with the mm source mapped by Beuther et al. (2002c). An outflow was detected by Beuther et al. (2002b) and S02 place an upper limit of $1 \mathrm{mJy}$ on the $3.6 \mathrm{~cm}$ radio continuum flux from any source in this region.

IRAS 19035+0641 (G40.622-0.137). This source is one of the $\mathrm{OH}$ maser sources mapped with the VLA by Argon et al. (2000). Several components, at different velocities in the range of 25 to $36 \mathrm{~km} \mathrm{~s}^{-1}$, were detected in the two main lines and spread over $\sim 1$ arcsec (Fig. 14 of Argon et al. 2000). Our Nançay-GBT observations show similar emission although the flux density of the components has varied since the Argon et al. observations. The flux density of the $1667 \mathrm{MHz}$ RHC compoment centered at velocity $27.39 \mathrm{~km} \mathrm{~s}^{-1}$ has rocketed up from 0.44 to $22.3 \mathrm{Jy}$. The IRAS source is also associated with $\mathrm{H}_{2} \mathrm{O}$ and $\mathrm{CH}_{3} \mathrm{OH}$ masers ( $\mathrm{S} 02$, SHK2000 and references therein). The $\mathrm{H}_{2} \mathrm{O}$ and $\mathrm{CH}_{3} \mathrm{OH}$ masers are coincident with a mid-infrared and $1.2 \mathrm{~mm}$ continuum emission sources (see Fig. 1 of Beuther et al. 2002a). The UCHII region, detected at 6-cm by Hughes \& Macleod (1993) and 3.6-cm by S02, shows 


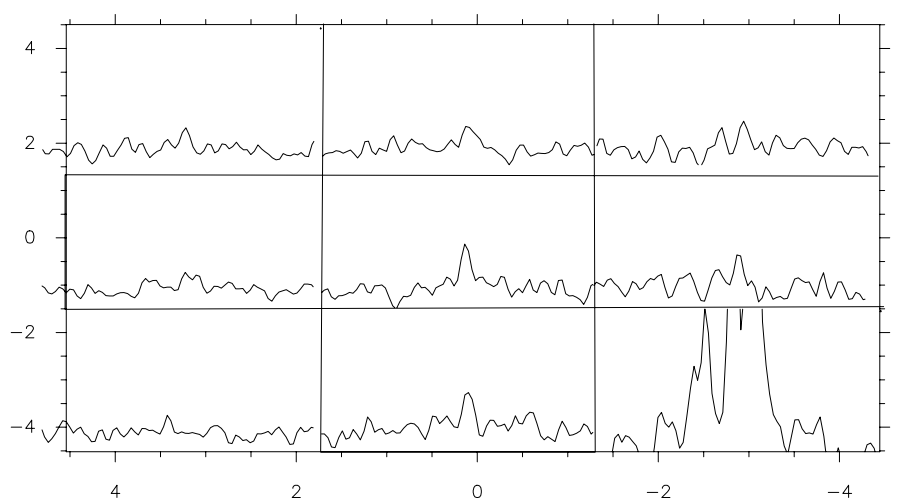

Fig. A.1. The spectra from the IRAS $19220+1432$ map in the $1720 \mathrm{MHz}$ $\mathrm{OH}$ maser. The $\mathrm{OH}$ maser associated with the IRAS position (middle panel) is contaminated by the strong $\mathrm{OH}$ maser source W51 which appears in the lower right hand side panel of the map. The axes show offsets in arcminutes from the IRAS position.

weak emission, of 3.8 and 4 mJy respectively. An outflow was detected towards this source by Beuther et al. (2002b).

IRAS 19092+0841. This source, also associated with $\mathrm{H}_{2} \mathrm{O}$ (P91) and $\mathrm{CH}_{3} \mathrm{OH}$ (SHK2000) masers, is one of the newly detected of $\mathrm{OH}$ masers. Radio continuum emission of 2.74 and $1.04 \mathrm{mJy}$ have been detected by Molinari et al. (1998) at the $2-$ and 6-cm wavelengths respectively.

IRAS 19118+0945. This is one of the sources with only $\mathrm{OH}$ masers. $\mathrm{No} \mathrm{H}_{2} \mathrm{O}$ or $\mathrm{CH}_{3} \mathrm{OH}$ masers have been detected towards this source (P91; SHK2000).

IRAS 19217+1651. This source is also associated with $\mathrm{CH}_{3} \mathrm{OH}$ masers and recently detected $\mathrm{H}_{2} \mathrm{O}$ masers as well as radio continuum emission ( $\mathrm{S} 02$ ). One mm source was detected by Beuther et al. (2002c) 5" north of the IRAS source and consistent with the $\mathrm{H}_{2} \mathrm{O}$ and $\mathrm{CH}_{3} \mathrm{OH}$ masers and a mid-infrared source. The radio continuum source is $\sim 5^{\prime \prime}$ west of the mm source. The detected $\mathrm{OH}$ maser emission seems not consistent with any of the previous sources, being located $\sim 1^{\prime}$ to the south. Beuther et al. (2004) studied this region in detail with high spatial resolution using Plateau de Bure Interferometer in the $\mathrm{CO} J=2-1$ and $\mathrm{SiO} J=2-1$ transitions. They conclude that the high-mass region IRAS $19217+1651$ exhibits a bipolar outflow and the region is dominated by the central driving source.

IRAS 19220+1432. The $\mathrm{OH}$ maser towards this source is contaminated by the nearby $\mathrm{OH}$ maser source W51. However the GBT map (Fig. 17) shows that there is a maser emission in the $1720-\mathrm{MHz}$ (RHC) line associated with the IRAS position. This is better illustrated in Fig. A.1 where the spectra from the map are plotted. The contamination of W51 maser is clear in the lower right corner of the map, but a component centred on the IRAS source is also visible. There is no other maser types associated with this source (S02). An flux density of $11 \mathrm{mJy}$ in the radio continuum was detected by $\mathrm{S} 02$ at $3.6-\mathrm{cm}$ wavelength.

IRAS 19374+2352. This source is also associated with a $\mathrm{H}_{2} \mathrm{O}$ maser (P91), but no $\mathrm{CH}_{3} \mathrm{OH}$ maser emission was detected by SHK2000. Free-free emission at $64.4 \mathrm{mJy}$ was detected by Molinari et al. (1998).

IRAS 19388+2357. This source was searched by Szymczak $\&$ Kus (2000) for $\mathrm{OH}$ maser but no emission was found. The observations here detect $\mathrm{OH}$ maser emission in the $1665-\mathrm{MHz}$ line spread over velocity range 34 to $39 \mathrm{~km} \mathrm{~s}^{-1}$. On the other hand, $\mathrm{CH}_{3} \mathrm{OH}$ maser emission, although detected by Schutte et al. (1993) in 1992 and Slysh et al. (1999) in 1995, was not detected in later observations by SHK2000 in 1999. This source is also associated with $\mathrm{H}_{2} \mathrm{O}$ maser (P91) and free-free emission (Molinari et al. 1998).

IRAS 19410+2336. This source is also associated with $\mathrm{H}_{2} \mathrm{O}$ and $\mathrm{CH}_{3} \mathrm{OH}$ masers ( $\mathrm{S} 02$; SHK2000). Near the $\mathrm{H}_{2} \mathrm{O}$ maser source, a radio continuum emission of $1 \mathrm{mJy}$ was detected by $\mathrm{S} 02$. Beuther et al. (2002c) $1.2-\mathrm{mm}$ continuum observations detect one source consistent with a mid-infrared source. The $1665-\mathrm{MHz} \mathrm{OH}$ maser emission detected close to the midinfrared source. An outflow was detected towards this source by Beuther et al. (2002b).

IRAS 20062+3550. This source was detected by Szymczak $\&$ Kus (2000) at the velocity of $-2.4 \mathrm{~km} \mathrm{~s}^{-1}$ in the $1665-\mathrm{MHz}$ line. The observations reported here detect a component in the same line over the velocity range -1 to $2.2 \mathrm{~km} \mathrm{~s}^{-1}$. Water and $\mathrm{CH}_{3} \mathrm{OH}$ masers have also been detected towards this source (P91; Slysh et al. 1999; SHK2000). No radio emission has been detected at $6 \mathrm{~cm}$ towards this source by Molinari et al. (1998). This source is one of sources studied in detail by Molinari et al. (2002) with the Owens Valley Radio Observatory (OVRO) millimeter wave array. Four distinct cores were identified in the $\mathrm{HCO}^{+} J=1-0$. Two of them are also detected in $\mathrm{H}^{13} \mathrm{CO}^{+}$ $J=1-0$. One core also has a $3.4 \mathrm{~mm}$ counterpart and is likely the most massive member of this cluster (Molinari et al. 2002).

IRAS 20126+4104. This source has a luminosity of $10^{4} L_{\odot}$ and is perhaps the best studied example of a massive protostar associated with a Keplerian disk and a jet/outflow system (Cesaroni et al. 1997, 1999; Hofner et al. 1999; Zhang et al. 1998; Cohen et al. 1988; Tofani et al. 1995; Moscadelli et al. 2000). The source is associated with $\mathrm{OH}, \mathrm{H}_{2} \mathrm{O}$ and $\mathrm{CH}_{3} \mathrm{OH}$ masers. Observations of the water masers using the VLA with angular resolution of $0.1^{\prime \prime}$ identified three emission regions (Tofani et al. 1995). Moscadelli et al. (2000) resolved two of these into 26 unresolved spots using the VLBA. The velocity and spatial structure of these spots were well fitted by a model with the spots arising at the interface between a jet and the surrounding molecular gas. Two features of $\mathrm{OH}$ masers were first detected in the 1665-MHz line by Cohen et al. (1988). More recently mapping of the $\mathrm{OH}$ and $\mathrm{CH}_{3} \mathrm{OH}$ masers at high angular resolution using MERLIN, Edris et al. (2005) showed that $\mathrm{OH}$ and methanol masers appear to trace part of the circumstellar disk around the central source.

IRAS 20188+3928. Only the $1720 \mathrm{MHz}$ of four $\mathrm{OH}$ lines is detected towards this source. The source is also associated with a $\mathrm{H}_{2} \mathrm{O}$ maser (P91) and a $2.86 \mathrm{Jy} 6 \mathrm{~cm}$ radio continuum source (Molinari et al. 1998).

IRAS 20227+4154. This source is associated with a $\mathrm{H}_{2} \mathrm{O}$ maser (P91) but no $\mathrm{CH}_{3} \mathrm{OH}$ maser emission was detected by SHK2000. Only the $1665 \mathrm{MHz} \mathrm{OH}$ maser line has been detected by our Nançay-GBT observations.

IRAS 22198+6336. This source is also associated with a $\mathrm{H}_{2} \mathrm{O}$ maser emission (P91) but no $\mathrm{CH}_{3} \mathrm{OH}$ maser emission was detected by SHK2000. No radio emission was detected by Molinari et al. (2002) at $6 \mathrm{~cm}$ wavelength. The two main lines of $\mathrm{OH}$ maser were detected by our Nançay-GBT observations.

IRAS 22272+6358. Although not detected in 1993 to a $3 \sigma$ upper limit of about $0.15 \mathrm{Jy}$ (Slysh et al. 1994), OH emission was found in 1999 in both main lines by Szymczak \& Kus (2000). This suggests considerable variations of the source. Approximately three years after Szymczak \& Kus observations, the Nançay-GBT observations show that the $\mathrm{OH}$ masers have varied. At the $1665 \mathrm{MHz}$ (LHC), a new component was detected centred at $8.46 \mathrm{~km} \mathrm{~s}^{-1}$ and the velocity of a bright component has slightly varied by $\sim 1.2 \mathrm{~km} \mathrm{~s}^{-1}$ (from -10.9 to $-12.12 \mathrm{~km} \mathrm{~s}^{-1}$ ). The velocity range of the $\mathrm{OH}$ emission is similar to that observed 
K. A. Edris et al.: OH maser survey towards HMPOs, Online Material p 39

for the $6.7 \mathrm{GHz}$ methanol maser ( $\mathrm{SHK} 2000)$. $\mathrm{No}_{2} \mathrm{O}$ maser was detected by P91.

IRAS 23139+5939. This sources is very similar to IRAS 18345-0641. Only the $1612 \mathrm{MHz}$ OH maser line has been detected and the source is also associated with $3.6-\mathrm{cm}$ radio continuum emission ( $\mathrm{S} 02$ ). It is also associated with a $\mathrm{H}_{2} \mathrm{O}$ maser (S02) which coincides with a mm continuum emission and midinfrared sources (Beuther et al. 2002a). An outflow along the line of sight was detected by Beuther et al. (2002b). 